\title{
Yang-Mills connections on nonorientable surfaces
}

\author{
Nan-Kuo Ho and Chiu-Chu Melissa Liu \\ Dedicated to the memory of Raoul Bott
}

In [1], Atiyah and Bott studied Yang-Mills functional over a Riemann surface from the point of view of Morse theory. We generalize their study to all closed, compact, connected, possibly nonorientable surfaces.

\section{Introduction}

Let $G$ be a compact, connected Lie group. In [1], Atiyah and Bott identified the affine space $\mathcal{A}$ of connections on a principal $G$-bundle $P$ over a Riemann surface with the affine space $\mathcal{C}$ of holomorphic structures on $P^{\mathbb{C}}=P \times_{G} G^{\mathbb{C}}$, where $G^{\mathbb{C}}$ is the complexification of $G$. The identification $\mathcal{A} \cong \mathcal{C}$ is an isomorphism of affine spaces, thus a diffeomorphism. It was conjectured in [1] that under this identification the Morse stratification of the Yang-Mills functional on $\mathcal{A}$ exists and coincides with the stratification of $\mathcal{C}$ from algebraic geometry $[14,25]$. The conjecture was proved by Daskalopoulos in [6] (see also [24] by Råde). The top stratum $\mathcal{C}_{s s}$ of $\mathcal{C}$ consists of semi-stable holomorphic structures on $P^{\mathbb{C}}$. Atiyah and Bott showed that the stratification of $\mathcal{C}$ is $\mathcal{G}^{\mathbb{C}}$-perfect, where $\mathcal{G}^{\mathbb{C}}=\operatorname{Aut}\left(P^{\mathbb{C}}\right)$. It has strong implications on the topology of the moduli space $\mathcal{M}(P)$ of $S$-equivalence classes of semi-stable holomorphic structures on $P^{\mathbb{C}}$. When $\mathcal{M}(P)$ is smooth, Atiyah and Bott found a complete set of generators of the cohomology groups $H^{*}(\mathcal{M}(P) ; \mathbb{Q})$ and recursive relations which determine the Poincaré polynomial $P_{t}(\mathcal{M}(P) ; \mathbb{Q})$. When $\mathcal{M}(P)$ is singular, their results give generators of the equivariant cohomology groups $H_{\mathcal{G}^{C}}^{*}\left(\mathcal{C}_{s s} ; \mathbb{Q}\right)$ and formula for the equivariant Poincaré series $P_{t}^{\mathcal{G}^{\mathcal{C}}}\left(\mathcal{C}_{s s} ; \mathbb{Q}\right)$.

Under the isomorphism $\mathcal{A} \cong \mathcal{C}$, the top stratum $\mathcal{C}_{s s}$ corresponds to $\mathcal{A}_{s s}$ which is the stable manifold of $\mathcal{N}_{s s}$, the set of central Yang-Mills connections, where the Yang-Mills functional achieves its absolute minimum $[1,6]$. 
When the absolute minimum is zero, $\mathcal{N}_{s s}$ is the set of flat connections (connections with zero curvature). By [6, Theorem C],

$$
\mathcal{M}(P) \cong \mathcal{N}_{s s} / \mathcal{G}
$$

where $\mathcal{G}=\operatorname{Aut}(P)$. So $\mathcal{M}(P)$ can be identified with the moduli space of gauge equivalence classes of central Yang-Mills connections on $P$. When the absolute minimum of the Yang-Mills functional is zero, or equivalently, the obstruction class $o(P) \in H^{2}\left(\Sigma, \pi_{1}(G)\right)$ is torsion, $\mathcal{M}(P)$ is the moduli space of gauge equivalence classes of flat connections on $P$. It is known that flat $G$-connections give rise to representations $\pi_{1}(\Sigma) \rightarrow G$, where $\pi_{1}(\Sigma)$ is the fundamental group of the base Riemann surface $\Sigma$ of $P$. More precisely,

$$
\bigcup_{\substack{P \in \operatorname{Prin}_{G}(\Sigma) \\ o(P) \text { torsion }}} \mathcal{M}(P)=\operatorname{Hom}\left(\pi_{1}(\Sigma), G\right) / G,
$$

where $G$ acts on the representation variety $\operatorname{Hom}\left(\pi_{1}(\Sigma), G\right)$ by conjugation. Yang-Mills $G$-connections (critical points of the Yang-Mills functional) give rise to representations $\Gamma_{\mathbb{R}}(\Sigma) \rightarrow G$, where $\Gamma_{\mathbb{R}}(\Sigma)$ is the central extension of $\pi_{1}(\Sigma)[1$, Section 6].

In this paper, we study the Yang-Mills functional on the space of connections on a principal $G$-bundle $P$ over a closed, compact, connected, nonorientable surface $\Sigma$. The pull back $\tilde{P}$ of $P$ to the orientable double cover $\pi: \tilde{\Sigma} \rightarrow \Sigma$ is always topologically trivial (Proposition 2.8), and $A \mapsto \pi^{*} A$ gives an inclusion from the space $\mathcal{A}$ of connections on $P$ into the space $\tilde{\mathcal{A}}$ of connections on $\tilde{P}$. The Yang-Mills functional on $\mathcal{A}$ is the restriction of that on $\tilde{\mathcal{A}}$. In the nonorientable case, the absolute minimum of the Yang-Mills functional is always zero, achieved by flat connections (see, for example [11]). We have

$$
\bigcup_{P \in \operatorname{Prin}_{G}(\Sigma)} \mathcal{M}(P)=\operatorname{Hom}\left(\pi_{1}(\Sigma), G\right) / G
$$

where $\mathcal{M}(P)$ is the moduli space of gauge equivalence classes of flat connections on $P$.

Let $\Sigma$ be a compact, connected, nonorientable surface without boundary. Then $\Sigma$ is diffeomorphic to the connected sum of $m>0$ copies of $\mathbb{R P}^{2}$, and the Euler characteristic $\chi(\Sigma)=2-m$. We derive the following results in this paper:

(i) We establish an exact correspondence between the gauge equivalence classes of Yang-Mills $G$-connections on $\Sigma$ and conjugacy classes of 
representations $\Gamma_{\mathbb{R}}(\Sigma) \rightarrow G$, where $\Gamma_{\mathbb{R}}(\Sigma)$ is the super central extension of $\pi_{1}(\Sigma)$ (Section 4).

(ii) We show that the moduli space of gauge equivalence classes of flat connections on any fixed principal $G$-bundle $P$ over $\Sigma$ is nonempty and connected if $\chi(\Sigma)<0$. This extends [11, Theorem 5.2] to the case $\Sigma=4 \mathbb{R P}^{2}$ (Section 5.4).

(iii) When $G=U(n)$, we give an explicit description of the $\mathcal{G}$-equivariant Morse stratification of the Yang-Mills functional, compute the Morse index of each stratum, and relate lower strata to top strata of spaces of $U(m)$-connections $(m<n)$ on $\Sigma$ and on its orientable double cover. This reduction also gives us a reduction of equivariant Poincaré series (Section 7).

We will describe the reduction (iii) for other classical groups in a subsequent work [12].

In the orientable case, the reduction (iii) and the understanding of the topology of the gauge group are sufficient to determine the equivariant Poincaré series of the top stratum recursively (by induction on dimension of the group $G$ ). In the nonorientable case, we need to compute the difference of the equivariant Morse and Poincare series; this difference vanishes in the orientable case due to equivariant perfectness of the stratification. We will address this in future works.

Using the Morse theory for the Yang-Mills functional over a closed (orientable or nonorientable) surface (studied in [1] and in this paper, respectively), D. Ramras proved an Atiyah-Segal theorem for surface groups in [26]: for any closed surface $\Sigma \neq S^{2}, \mathbb{R P}^{2}, K_{\text {def }}^{*}\left(\pi_{1}(\Sigma)\right) \cong K^{*}(\Sigma)$ for $*>0$ when $\Sigma$ is orientable, and for $* \geq 0$ when $\Sigma$ is nonorientable, where $K_{\text {def }}^{*}$ are Carlsson's deformation $K$-groups. Using algebraic topology methods, T. Baird computed the $S U(2)$-equivariant cohomology of $\operatorname{Hom}\left(\pi_{1}(\Sigma), S U(2)\right)$ and the ordinary cohomology of the quotient space $\operatorname{Hom}\left(\pi_{1}(\Sigma), S U(2)\right) /$ $S U(2)$ for any closed nonorientable surface $\Sigma[4]$.

For the purpose of Morse theory, we should consider the Sobolev space of $L_{k-1}^{2}$ connections $\mathcal{A}^{k-1}$ and the group of $L_{k}^{2}$ gauge transformations $\mathcal{G}^{k}$ and $\left(\mathcal{G}^{\mathbb{C}}\right)^{k}$, where $k \geq 2$. We will not emphasize the regularity issues through out the paper, but refer the reader to [1, Section 14] and [6] for details.

We now give a clear description of the remaining sections. In Section 2, we review various representation varieties of flat connections, and show that the pull-back of any principal $G$-bundle over a nonorientable surface to its orientable double cover is topologically trivial. In Section 3, we review definitions of the Yang-Mills functional and Yang-Mills connections over 
an orientable surface, and give corresponding definitions for a nonorientable surface. We describe involutions on the principal $G$-bundles and on the space of connections induced by the deck transformation on the orientable double cover of the nonorientable surface. Section 4 contains our main construction and justification. We introduce the super central extension of the fundamental group of a surface; it is the central extension if and only if the surface is orientable. We establish a precise correspondence between Yang-Mills connections and representations of the super central extension. We introduce representation varieties for Yang-Mills connections, and describe an involution on symmetric representation varieties induced by the deck transformation on the orientable double cover. We also introduce extended moduli spaces for nonorientable surfaces. In Section 5, we discuss the $\mathcal{G}$-equivariant Morse stratification and reduction for general compact connected Lie groups. As a byproduct, we reproduce and extend the results on connected components of the moduli space of flat connections over closed nonorientable surfaces $[10,11]$. We specialize to the case $G=U(n)$ in Section 6 (orientable case) and Section 7 (nonorientable case). We give explicit description of the $\mathcal{G}$-equivariant Morse stratification of the space of connections. The main reference of Section 6 of this paper is [1]. In [1, Section 7], the reduction is derived at the level of strata, which are infinite dimensional manifolds. Knowing the exact correspondence between Yang-Mills connections and representations, we work mainly at the level of representation varieties which are finite dimensional.

\section{Flat connections and representations}

Let $G$ be a compact, connected Lie group, and let $P$ be a principal $G$ bundle on a closed, compact, connected surface $\Sigma$. We say a connection $A \in \mathcal{A}(P)$ is flat if its curvature vanishes. Let $\mathcal{N}_{0}(P) \subset \mathcal{A}(P)$ be the set of flat connections on $P$. Note that when $\Sigma$ is orientable, $\mathcal{N}_{0}(P)$ can be empty.

\subsection{Representation varieties of flat connections}

We first introduces some notation similar to that in [11, Section 2.3]. Let $\Sigma_{0}^{\ell}$ be the closed, compact, connected, orientable surface with $\ell \geq 0$ handles. Let $\Sigma_{1}^{\ell}$ be the connected sum of $\Sigma_{0}^{\ell}$ and $\mathbb{R P}^{2}$, and let $\Sigma_{2}^{\ell}$ be the connected sum of $\Sigma_{0}^{\ell}$ and a Klein bottle. Any closed, compact, connected surface is of the form $\Sigma_{i}^{\ell}$, where $\ell$ is a nonnegative integer, $i=0,1,2 . \Sigma_{i}^{\ell}$ is orientable if and only if $i=0$. Use 1 as the identity of $\pi_{1}(\Sigma)$ and $e$ as the identity of $G$. 
We have

$$
\begin{aligned}
& \pi_{1}\left(\Sigma_{0}^{\ell}\right)=\left\langle A_{1}, B_{1}, \ldots, A_{\ell}, B_{\ell} \mid \prod_{i=1}^{\ell}\left[A_{i}, B_{i}\right]=1\right\rangle, \\
& \pi_{1}\left(\Sigma_{1}^{\ell}\right)=\left\langle A_{1}, B_{1}, \ldots, A_{\ell}, B_{\ell}, C \mid \prod_{i=1}^{\ell}\left[A_{i}, B_{i}\right]=C^{2}\right\rangle, \\
& \pi_{1}\left(\Sigma_{2}^{\ell}\right)=\left\langle A_{1}, B_{1}, \ldots, A_{\ell}, B_{\ell}, D, C \mid \prod_{i=1}^{\ell}\left[A_{i}, B_{i}\right]=C D C^{-1} D\right\rangle .
\end{aligned}
$$

It is known that a flat connection gives rise to a homomorphism $\pi_{1}(\Sigma) \rightarrow$ $G$. Introduce representation varieties

$$
\begin{aligned}
& X_{\text {flat }}^{\ell, 0}(G)=\left\{\left(a_{1}, b_{1}, \ldots, a_{\ell}, b_{\ell}\right) \in G^{2 \ell} \mid \prod_{i=1}^{\ell}\left[a_{i}, b_{i}\right]=e\right\}, \\
& X_{\text {flat }}^{\ell, 1}(G)=\left\{\left(a_{1}, b_{1}, \ldots, a_{\ell}, b_{\ell}, c\right) \in G^{2 \ell+1} \mid \prod_{i=1}^{\ell}\left[a_{i}, b_{i}\right]=c^{2}\right\}, \\
& X_{\text {flat }}^{\ell, 2}(G)=\left\{\left(a_{1}, b_{1}, \ldots, a_{\ell}, b_{\ell}, d, c\right) \in G^{2 \ell+2} \mid \prod_{i=1}^{\ell}\left[a_{i}, b_{i}\right]=c d c^{-1} d\right\} .
\end{aligned}
$$

Then

$$
\bigcup_{P \in \operatorname{Prin}_{G}\left(\Sigma_{i}^{\ell}\right)} \mathcal{N}_{0}(P) / \mathcal{G}_{0}(P) \cong \operatorname{Hom}\left(\pi_{1}\left(\Sigma_{i}^{\ell}\right), G\right) \cong X_{\text {flat }}^{\ell, i}(G)
$$

for $i=0,1,2, \ell \geq 0$, where $\mathcal{G}_{0}(P)$ is the based gauge group which consists of gauge transformations on $G$ that take value the identity $e \in G \cong \operatorname{Aut}\left(P_{x_{0}}\right)$ at a fixed point of $x_{0} \in \Sigma_{i}^{\ell}$. The action of $\mathcal{G}_{0}(P)$ on $\mathcal{N}_{0}(P)$ is free. Let $\mathcal{G}(P)=\operatorname{Aut}(P)$ be the gauge group. Then $\mathcal{G}_{0}(P)$ is a normal subgroup of $\mathcal{G}(P)$, and

$$
G=\mathcal{G}(P) / \mathcal{G}_{0}(P)
$$

\section{Example 2.1.}

$$
\begin{aligned}
X_{\text {flat }}^{\ell, 0}(U(1))= & \left\{\left(a_{1}, b_{1}, \ldots, a_{\ell}, b_{\ell}\right) \in U(1)^{2 \ell}\right\}=U(1)^{2 \ell} \\
X_{\text {flat }}^{\ell, 1}(U(1))= & \left\{\left(a_{1}, b_{1}, \ldots, a_{\ell}, b_{\ell}, c\right) \in U(1)^{2 \ell+1} \mid c^{2}=1\right\}=U(1)^{2 \ell} \times\{ \pm 1\}, \\
X_{\text {flat }}^{\ell, 2}(U(1))= & \left\{\left(a_{1}, b_{1}, \ldots, a_{\ell}, b_{\ell}, d, c\right) \in U(1)^{2 \ell+2} \mid d^{2}=1\right\}=U(1)^{2 \ell+1} \\
& \times\{ \pm 1\} .
\end{aligned}
$$


Let $G$ act on $G^{2 \ell+i}$ by

$$
g \cdot\left(c_{1}, \ldots, c_{2 \ell+i}\right)=\left(g c_{1} g^{-1}, \ldots, g c_{2 \ell+i} g^{-1}\right)
$$

This action preserves the subset $X_{\text {flat }}^{\ell, i}(G) \subset G^{2 \ell+i}$, so $G$ acts on $X_{\text {flat }}^{\ell, i}(G)$, and

$$
\bigcup_{P \in \operatorname{Prin}_{G}\left(\Sigma_{i}^{\ell}\right)} \mathcal{N}_{0}(P) / \mathcal{G}(P) \cong \operatorname{Hom}\left(\pi_{1}\left(\Sigma_{i}^{\ell}\right), G\right) / G \cong X_{\text {flat }}^{\ell, i}(G) / G
$$

is the moduli space of gauge equivalence classes of flat $G$-connections on $\Sigma_{i}^{\ell}$. We also have homotopy equivalences

$$
\bigcup_{P \in \operatorname{Prin}_{G}\left(\Sigma_{i}^{\ell}\right)} \mathcal{N}_{0}(P)^{h \mathcal{G}(P)} \sim \operatorname{Hom}\left(\pi_{1}\left(\Sigma_{i}^{\ell}\right), G\right)^{h G} \sim X_{\text {flat }}^{\ell, i}(G)^{h G}
$$

where $X^{h G}$ denotes the homotopic orbit space $E G \times_{G} X$.

Notation 2.2. In the rest of this paper, we will use the following notation:

Denote the $2 \ell$-vector by $V=\left(a_{1}, b_{1}, \ldots, a_{\ell}, b_{\ell}\right) \in G^{2 \ell}$. Define $\mathfrak{m}(V)$ and $\mathfrak{r}(V)$ by

$$
\begin{gathered}
\mathfrak{m}(V)=\prod_{i=1}^{\ell}\left[a_{i}, b_{i}\right], \\
\mathfrak{r}(V)=\left(b_{\ell}, a_{\ell}, \ldots, b_{1}, a_{1}\right) .
\end{gathered}
$$

Then $\mathfrak{m}(\mathfrak{r}(V))=\mathfrak{m}(V)^{-1}$. Given $g \in G$, define

$$
g V g^{-1}=\left(g a_{1} g^{-1}, g b_{1} g^{-1}, \ldots, g a_{\ell} g^{-1}, g b_{\ell} g^{-1}\right)
$$

With the above notation, the representation varieties $X_{\text {flat }}^{\ell, i}(G)$ can be written as follows:

$$
\begin{aligned}
& X_{\text {flat }}^{\ell, 0}(G)=\left\{V \in G^{2 \ell} \mid \mathfrak{m}(V)=e\right\} \\
& X_{\text {flat }}^{\ell, 1}(G)=\left\{(V, c) \mid V \in G^{2 \ell}, c \in G, \mathfrak{m}(V)=c^{2}\right\} \\
& X_{\text {flat }}^{\ell, 2}(G)=\left\{(V, d, c) \mid V \in G^{2 \ell}, d, c \in G, \mathfrak{m}(V)=c d c^{-1} d\right\} .
\end{aligned}
$$




\subsection{Symmetric representation varieties of flat connections}

Let $\Sigma$ be a closed, compact, connected, nonorientable surface, and let $\pi$ : $\tilde{\Sigma} \rightarrow \Sigma$ be the orientable double cover. The goal of this and the next subsection is to relate the representation varieties of $\Sigma$ to those of $\tilde{\Sigma}$.

Let $\Sigma=\Sigma_{i}^{\ell}$, where $i=1,2$. Then $\Sigma$ is homeomorphic to the connected sum of $2 \ell+i$ copies of $\mathbb{R P}^{2}$, and its orientable double cover $\tilde{\Sigma}$ is $\Sigma_{0}^{2 \ell+i-1}$, a Riemann surface of genus $2 \ell+i-1$.

In the rest of this subsection, we follow [8, Section 5] closely. Define

$$
\begin{aligned}
Z_{\text {flat }}^{\ell, 1}(G)= & \left\{(V, c, \bar{V}, \bar{c}) \mid V, \bar{V} \in G^{2 \ell}, c, \bar{c} \in G, \mathfrak{m}(V)=c \bar{c}, \mathfrak{m}(\bar{V})=\bar{c} c\right\} \\
Z_{\text {flat }}^{\ell, 2}(G)= & \left\{(V, d, c, \bar{V}, \bar{d}, \bar{c}) \mid V, \bar{V} \in G^{2 \ell}, d, c, \bar{d}, \bar{c} \in G, \mathfrak{m}(V)=c \bar{d} c^{-1} d,\right. \\
& \left.\mathfrak{m}(\bar{V})=\bar{c} d \bar{c}^{-1} \bar{d}\right\}
\end{aligned}
$$

Lemma 2.3. For $i=1,2$, define $\Phi_{G}^{\ell, i}: G^{2(2 \ell+i)} \rightarrow G^{2(2 \ell+i-1)}$ by

$$
\begin{aligned}
\Phi_{G}^{\ell, 1}(V, c, \bar{V}, \bar{c}) & =\left(V, c \mathfrak{r}(\bar{V}) c^{-1}\right), \\
\Phi_{G}^{\ell, 2}(V, d, c, \bar{V}, \bar{d}, \bar{c}) & =\left(V, d^{-1} c \mathfrak{r}(\bar{V}) c^{-1} d, d^{-1}, c \bar{c}\right),
\end{aligned}
$$

where $V, \bar{V} \in G^{2 \ell}, c, d, \bar{c}, \bar{d} \in G$. Then

$$
\Phi_{G}^{\ell, i}\left(Z_{\text {flat }}^{\ell, i}(G)\right)=X_{\text {flat }}^{2 \ell+i-1,0}(G) .
$$

Proof. It is straightforward to check $\Phi_{G}^{\ell, i}\left(Z_{\text {flat }}^{\ell, i}(G)\right) \subset X_{\text {flat }}^{2 \ell+i-1,0}(G)$. It remains to show that $X_{\text {flat }}^{2 \ell+i-1,0}(G) \subset \Phi_{G}^{\ell, i}\left(Z_{\text {flat }}^{\ell, i}(G)\right)$.

1. $X_{\text {flat }}^{2 \ell, 0}(G) \subset \Phi_{G}^{\ell, 1}\left(Z_{\text {flat }}^{\ell, 1}(G)\right)$ : Given $\left(V_{1}, V_{2}\right) \in X_{\text {flat }}^{2 \ell, 0}(G)$, where $V_{1}, V_{2} \in$ $G^{2 \ell}$, we have $\mathfrak{m}\left(V_{1}\right) \mathfrak{m}\left(V_{2}\right)=e$. Let

$$
\bar{c}=\mathfrak{m}\left(V_{1}\right)=\mathfrak{m}\left(V_{2}\right)^{-1}=\mathfrak{m}\left(\mathfrak{r}\left(V_{2}\right)\right)
$$

Then

$$
\left(V_{1}, e, \mathfrak{r}\left(V_{2}\right), \bar{c}\right) \in Z_{\text {flat }}^{\ell, 1}(G), \quad\left(V_{1}, V_{2}\right)=\Phi_{G}^{\ell, 1}\left(V_{1}, e, \mathfrak{r}\left(V_{2}\right), \bar{c}\right)
$$

2. $X_{\text {flat }}^{2 \ell+1,0}(G) \subset \Phi_{G}^{\ell, 2}\left(Z_{\text {flat }}^{\ell, 2}(G)\right)$ : Given $\left(V_{1}, V_{2}, a, b\right) \in X_{\text {flat }}^{2 \ell+1,0}(G)$, where $V_{1}, V_{2} \in G^{2 \ell}$ and $a, b \in G$, we have $\mathfrak{m}\left(V_{1}\right) \mathfrak{m}\left(V_{2}\right)[a, b]=e$. Let

$$
d=a^{-1}, \quad \bar{d}=a \mathfrak{m}\left(V_{1}\right), \quad c=a^{-1}, \quad \bar{c}=a b .
$$


Then

$$
\left(V_{1}, d, c, \mathfrak{r}\left(V_{2}\right), \bar{d}, \bar{c}\right) \in Z_{\text {flat }}^{\ell, 2}(G), \quad\left(V_{1}, V_{2}, a, b\right)=\Phi_{G}^{\ell, 2}\left(V_{1}, d, c, \mathfrak{r}\left(V_{2}\right), \bar{d}, \bar{c}\right) .
$$

Let $G^{2}$ act on $Z_{\text {flat }}^{\ell, 1}(G), Z_{\text {flat }}^{\ell, 2}(G)$ by

$$
\begin{aligned}
\left(g_{1}, g_{2}\right) \cdot(V, c, \bar{V}, \bar{c}) & =\left(g_{1} V g_{1}^{-1}, g_{1} c g_{2}^{-1}, g_{2} \bar{V} g_{2}^{-1}, g_{2} \bar{c} g_{1}^{-1}\right) \\
\left(g_{1}, g_{2}\right) \cdot(V, d, c, \bar{V}, \bar{d}, \bar{c}) & =\left(g_{1} V g_{1}^{-1}, g_{1} d g_{1}^{-1}, g_{1} c g_{2}^{-1}, g_{2} \bar{V} g_{2}^{-1}, g_{2} \bar{d} g_{2}^{-1} g_{2} \bar{c} g_{1}^{-1}\right)
\end{aligned}
$$

respectively, where $V, \bar{V} \in G^{2 \ell}$ and $g_{1}, g_{2}, c, \bar{c}, d, \bar{d} \in G$.

Lemma 2.4. The surjection $\Phi_{G}^{\ell, i}: Z_{\text {flat }}^{\ell, i}(G) \rightarrow X_{\text {flat }}^{2 \ell+i-1,0}(G)$ induces homeomorphisms

$$
Z_{\text {flat }}^{\ell, i}(G) / G^{2} \cong X_{\text {flat }}^{2 \ell+i-1,0}(G) / G \cong \operatorname{Hom}\left(\pi_{1}\left(\Sigma_{0}^{2 \ell+i-1}\right), G\right) / G
$$

and a homotopy equivalence

$$
Z_{\text {flat }}^{\ell, i}(G)^{h G^{2}} \sim X_{\text {flat }}^{2 \ell+i-1,0}(G)^{h G},
$$

where $X^{h G}$ denotes the homotopic orbit space $E G \times_{G} X$.

Proof. The case $i=1$ of (2.3) was proved in [8]; the case $i=2$ is similar.

To see (2.4), let $G_{1}=G \times\{1\} \subset G \times G$ and $G_{2}=\{1\} \times G \subset G \times G$, so that $G_{1} \cong G_{2} \cong G$, and the $G_{1}$-action and $G_{2}$-action on $Z_{\text {flat }}^{\ell, i}(G)$ commute. Note that $G_{2}$ is a closed normal subgroup of $G \times G$ and that $G_{2}$ acts on $Z_{\text {flat }}^{\ell, i}(G)$ freely, so the natural projection $Z_{\text {flat }}^{\ell, i}(G) \rightarrow Z_{\text {flat }}^{\ell, i}(G) / G_{2}$ induces a homotopy equivalence

$$
Z_{\text {flat }}^{\ell, i}(G)^{h(G \times G)} \sim\left(Z_{\text {flat }}^{\ell, i}(G) / G_{2}\right)^{h G_{1}}
$$

It is straightforward to check that the surjection $\Phi_{G}^{\ell, i}: Z_{\text {flat }}^{\ell, i}(G) \rightarrow X_{\text {flat }}^{2 \ell+i-1,0}(G)$ descends to a homeomorphism $\bar{\Phi}_{G}^{\ell, i}: Z_{\text {flat }}^{\ell, i}(G) / G_{2} \rightarrow X_{\text {flat }}^{2 \ell+i-1,0}(G)$. Moreover, $\bar{\Phi}_{G}^{\ell, i}$ is $G$-equivariant with respect to the $G_{1}$-action on $Z_{\text {flat }}^{\ell, i}(G) / G_{2}$ and 
the $G$-action on $X_{\text {flat }}^{2 \ell+i-1,0}(G)$, so $\bar{\Phi}_{G}^{\ell, i}$ induces a homotopy equivalence

$$
\left(Z_{\text {flat }}^{\ell, i}(G) / G_{2}\right)^{h G_{1}} \sim X_{\text {flat }}^{2 \ell+i-1,0}(G)^{h G} .
$$

By [11, Theorem 3.3], when $\ell>0$ there is a bijection

$$
\pi_{0}\left(\operatorname{Hom}\left(\pi_{1}\left(\Sigma_{0}^{\ell}\right), G\right) / G\right) \longrightarrow \pi_{1}\left(G_{s s}\right)
$$

where $G_{s s}=[G, G]$ is the maximal connected semi-simple subgroup of $G$. Since $G^{2}$ and $G$ are connected, we conclude that

Corollary 2.5. Suppose that $i=1,2$ and $\ell \geq 0$. Then when $(\ell, i) \neq(0,1)$ there is a bijection

$$
\pi_{0}\left(Z_{\text {flat }}^{\ell, i}(G)\right) \longrightarrow \pi_{1}\left(G_{s s}\right)
$$

\subsection{Involution on symmetric representation varieties of flat connections}

In this section, $i=1,2$.

Let $\tau: Z_{\text {flat }}^{\ell, i}(G) \rightarrow Z_{\text {flat }}^{\ell, i}(G)$ be the involution defined in [8]:

$$
\tau(V, v, \bar{V}, \bar{v})=(\bar{V}, \bar{v}, V, v)
$$

where $V, \bar{V} \in G^{2 \ell}$ and $v, \bar{v} \in G^{i}$. There is an injection $I: X_{\text {flat }}^{\ell, i}(G) \rightarrow Z_{\text {flat }}^{\ell, i}(G)$ given by $(V, v) \mapsto(V, v, V, v)$ such that

$$
I\left(X_{\text {flat }}^{\ell, i}(G)\right)=Z_{\text {flat }}^{\ell, i}(G)^{\tau}
$$

where $Z_{\text {flat }}^{\ell, i}(G)^{\tau}$ is the fixed locus of the involution $\tau$. We will show that $Z_{\text {flat }}^{\ell, i}(G)^{\tau}$ corresponds to topologically trivial flat $G$-bundles over the Riemann surface $\Sigma_{0}^{2 \ell+i-1}$. To do so, we first recall the definition of the obstruction map, which detects the topological type of a flat $G$-bundle.

Let $H$ be the connected component of the identity of the center of $G$, and let $G_{s s}=[G, G]$ be the commutator group. Then $H$ is a compact torus, and $G_{s s}$ is the maximal connected semi-simple subgroup of $G$. Let $\rho_{s s}$ : $\tilde{G}_{s s} \rightarrow G_{s s}$ and $\rho: \tilde{G} \rightarrow G$ be universal coverings. Then $\tilde{G}=\mathfrak{h} \times \tilde{G}_{s s}$, where 
$\mathfrak{h}=\operatorname{Lie}(H)$. Define $\rho^{2 \ell}: \tilde{G}^{2 \ell} \rightarrow G^{2 \ell}$ by

$$
\rho^{2 \ell}\left(\tilde{a}_{1}, \tilde{b}_{1}, \ldots, \tilde{a}_{\ell}, \tilde{b}_{\ell}\right) \longmapsto\left(\rho\left(\tilde{a}_{1}\right), \rho\left(\tilde{b}_{1}\right), \ldots, \rho\left(\tilde{a}_{\ell}\right), \rho\left(\tilde{b}_{\ell}\right)\right)
$$

With the above notation, the obstruction map $o: X_{\text {flat }}^{\ell, 0}(G) \rightarrow \operatorname{Ker}\left(\rho_{s s}\right) \cong$ $\pi_{1}\left(G_{s s}\right)$ is defined as follows: given $V \in G^{2 \ell}$, pick $\tilde{V} \in G^{2 \ell}$ such that $\rho^{2 \ell}(\tilde{V})$ $=V$, and define $o(V)=\mathfrak{m}(\tilde{V})$. Then $o(V) \in \operatorname{Ker} \rho \cap G_{s s}=\operatorname{Ker} \rho_{s s}$, and the definition is independent of choice of $\tilde{V}$. The flat $G$-bundle associated to $V$ is topologically trivial if and only if $o(V)=\tilde{e}$, where $\tilde{e}$ is the identity element of $\tilde{G}$.

Let $o: X_{\text {flat }}^{2 \ell+i-1,0}(G) \rightarrow \operatorname{Ker}\left(\rho_{s s}\right)$ be the obstruction map, and let

$$
o^{\prime}=o \circ \Phi_{G}^{\ell, i}: Z_{\text {flat }}^{\ell, i}(G) \longrightarrow \pi_{1}\left(G_{s s}\right)
$$

Let $e$ be the identity element of $G$.

Lemma 2.6. $o^{\prime}(\tau(y))=o^{\prime}(y)^{-1}$ for $y \in Z_{\text {flat }}^{\ell, i}(G)$.

Proof. We will prove the case $i=1$. The case $i=2$ is similar.

Given $y=(V, c, \bar{V}, \bar{c}) \in Z_{\text {flat }}^{\ell, 1}(G)$, where $V, \bar{V} \in G^{2 \ell}$ and $c, \bar{c} \in G$, pick $V^{\prime}, \bar{V}^{\prime} \in \tilde{G}^{2 \ell}$ and $c^{\prime}, \bar{c}^{\prime} \in \tilde{G}$ such that $\rho^{2 \ell}\left(V^{\prime}\right)=V, \rho^{2 \ell}\left(\bar{V}^{\prime}\right)=\bar{V}, \rho\left(c^{\prime}\right)=c$, and $\rho\left(\bar{c}^{\prime}\right)=\bar{c}$. Then

$$
\rho\left(\mathfrak{m}\left(V^{\prime}\right)\left(c^{\prime} \bar{c}^{\prime}\right)^{-1}\right)=\mathfrak{m}(V)(c \bar{c})^{-1}=e, \quad \rho\left(\mathfrak{m}\left(\bar{V}^{\prime}\right)\left(\bar{c}^{\prime} c^{\prime}\right)^{-1}\right)=\mathfrak{m}(\bar{V})(\bar{c} c)^{-1}=e
$$

Let $k=\mathfrak{m}\left(V^{\prime}\right)\left(c^{\prime} \bar{c}^{\prime}\right)^{-1}$ and $\bar{k}=\mathfrak{m}\left(\bar{V}^{\prime}\right)\left(\bar{c}^{\prime} c^{\prime}\right)^{-1}$. Then $k, \bar{k} \in \operatorname{Ker} \rho \subset Z(\tilde{G})$. We have

$$
\begin{aligned}
o^{\prime}(y) & =o^{\prime}(V, c, \bar{V}, \bar{c})=o\left(V, c \mathfrak{r}(\bar{V}) c^{-1}\right)=\mathfrak{m}\left(V^{\prime}\right) \mathfrak{m}\left(c^{\prime} \mathfrak{r}\left(\bar{V}^{\prime}\right)\left(c^{\prime}\right)^{-1}\right) \\
& =\mathfrak{m}\left(V^{\prime}\right) c^{\prime} \mathfrak{m}\left(\bar{V}^{\prime}\right)^{-1}\left(c^{\prime}\right)^{-1}=\left(k c^{\prime} \bar{c}^{\prime}\right) c^{\prime}\left(\bar{k} \bar{c}^{\prime} c^{\prime}\right)^{-1}\left(c^{\prime}\right)^{-1}=k \bar{k}^{-1}, \\
o^{\prime}(\tau(y)) & =o^{\prime}(\bar{V}, \bar{c}, V, c)=\bar{k} k^{-1} .
\end{aligned}
$$

So $o^{\prime}(\tau(y))=o^{\prime}(y)^{-1}$.

Lemma 2.7. $o^{\prime} \circ I(x)=\tilde{e}$ for all $x \in X_{\text {flat }}^{\ell, i}(G)$.

Proof. We will prove the case $i=1$. The case $i=2$ is similar.

Given $(V, c) \in X_{\text {flat }}^{\ell, 1}(G)$, where $V \in G^{2 \ell}$ and $c \in G$, pick $\tilde{V} \in \tilde{G}^{2 \ell}$ and $\tilde{c} \in$ $\tilde{G}$ such that $\rho^{2 \ell}(\tilde{V})=V$ and $\rho(\tilde{c})=c$. Then $\rho\left(\mathfrak{m}(\tilde{V}) \tilde{c}^{-2}\right)=\mathfrak{m}(V) c^{-2}=e$. 
Let $k=\mathfrak{m}(\tilde{V}) \tilde{c}^{-2} \in \operatorname{Ker} \rho \subset Z(\tilde{G})$. Then

$$
\begin{aligned}
o^{\prime} \circ I(V, c) & =o^{\prime}(V, c, V, c)=o\left(V, c \mathfrak{r}(V) c^{-1}\right)=\mathfrak{m}(\tilde{V}) \mathfrak{m}\left(\tilde{c} \mathfrak{r}(\tilde{V}) \tilde{c}^{-1}\right) \\
& =\mathfrak{m}(\tilde{V}) \tilde{c} \mathfrak{m}(\tilde{V})^{-1} \tilde{c}^{-1}=k \tilde{c}^{2} \cdot \tilde{c}\left(k \tilde{c}^{2}\right)^{-1} \tilde{c}^{-1}=\tilde{e}
\end{aligned}
$$

By [11, Theorem 5.2], any topological principal $G$-bundle on a closed, connected, nonorientable surface admits a flat connection. By Lemma 2.7, the pullback of a flat $G$-bundle over $\Sigma_{i}^{\ell}$ under the orientable double cover $\Sigma_{0}^{2 \ell+i-1} \rightarrow \Sigma_{i}^{\ell}$ is a topologically trivial flat $G$-bundle over $\Sigma_{0}^{2 \ell+i-1}$. We conclude that:

Proposition 2.8. Let $G$ be a compact, connected Lie group. Let $\Sigma$ be a closed, connected, nonorientable surface, and let $\pi: \tilde{\Sigma} \rightarrow \Sigma$ be the orientable double cover. Then the pullback $\pi^{*} P$ of any topological principal $G$-bundle $P \rightarrow \Sigma$ is topologically trivial.

\section{Yang-Mills functional and Yang-Mills connections}

In this section, we will define Yang-Mills functional and Yang-Mills connections on nonorientable closed surfaces.

\subsection{Yang-Mills functional and Yang-Mills connections on orientable surfaces}

We first recall the Yang-Mills functional and Yang-Mills connections on orientable closed surfaces, following [1].

Let $G$ be a compact connected Lie group. Let $\Sigma$ be a Riemann surface. There is a unique Kähler metric $h$ such that the scalar curvature is a constant and the Kähler form $\omega$ is the unique harmonic 2 -form on $\Sigma$ such that $\int_{\Sigma} \omega=$ 1. We call it the canonical metric of the Riemann surface.

Let $\mathcal{A}(P)$ denote the space of $C^{\infty}$ connections on $P$. Then $\mathcal{A}(P)$ is an affine space whose associated (real) vector space is $\Omega^{1}(\Sigma, \operatorname{ad}(P))$. The Yang-Mills functional $L: \mathcal{A}(P) \rightarrow \mathbb{R}$ is defined by

$$
L(A)=\int_{\Sigma} \operatorname{Tr}(F(A) \wedge * F(A)),
$$

where $F(A)$ is the curvature form of $A$. 
Let $A_{t}=A+t \eta$ be a line of connections, where $\eta \in \Omega^{1}(\Sigma, \operatorname{ad}(P))$. Then

$$
F\left(A_{t}\right)=F(A)+t d_{A} \eta+\frac{1}{2} t^{2}[\eta, \eta]
$$

so

$$
\begin{aligned}
L\left(A_{t}\right) & =L(A)+2 t \int_{\Sigma} \operatorname{Tr}\left(d_{A} \eta \wedge * F(A)\right)+O\left(t^{2}\right) \\
& =L(A)+2 t \int_{\Sigma} \operatorname{Tr}\left(\eta \wedge * d_{A} * F(A)\right)+O\left(t^{2}\right)
\end{aligned}
$$

So $A$ is a critical point of $L$ iff it satisfies the Yang-Mills equation:

$$
d_{A}^{*} F(A)=* d_{A} * F(A)=0 .
$$

We call critical points Yang-Mills connections on $P$. Note that flat connections are Yang-Mills connections.

\subsection{Involution on the principal bundle}

Let $\Sigma$ be a connected, nonorientable, closed surface. Then $\Sigma$ is diffeomorphic to the connected sum of $m>0$ copies of $\mathbb{R P}^{2}$ 's. Let $\pi: \tilde{\Sigma} \rightarrow \Sigma$ be the orientable double cover, and let $\tau: \tilde{\Sigma} \rightarrow \tilde{\Sigma}$ be the deck transformation. Then $\tilde{\Sigma}$ is a Riemann surface of genus $m-1$, and $\tau$ is an anti-holomorphic, anti-symplectic involution with no fixed point.

Let $P \rightarrow \Sigma$ be a principal $G$-bundle. Let $\tilde{P}=\pi^{*} P$ be the pullback principal $G$-bundle on $\tilde{\Sigma}$. By Proposition 2.8, $\tilde{P}$ is topologically trivial. There is an involution $\tilde{\tau}: \tilde{P} \rightarrow \tilde{P}$ which is $G$-equivariant and covers $\tau: \tilde{\Sigma} \rightarrow \tilde{\Sigma}$.

More explicitly, fix a trivialization $\tilde{P} \cong \tilde{\Sigma} \times G$. The right $G$-action on $\tilde{P}$ is given by

$$
(x, h) \cdot g=(x, h \cdot g)
$$

where $g \in G,(x, h) \in \tilde{\Sigma} \times G$. It is straightforward to check that

$$
(x, h) \cdot\left(g_{1} g_{2}\right)=\left((x, h) \cdot g_{1}\right) \cdot g_{2} .
$$

The involution $\tilde{\tau}$ is $G$-equivariant with respect to the above $G$-action:

$$
\tilde{\tau}(x, h) \cdot g=\tilde{\tau}((x, h) \cdot g)
$$

for $(x, h) \in \tilde{P}, g \in G$. Let $s: \tilde{\Sigma} \rightarrow G$ be defined by

$$
\tilde{\tau}(x, e)=(\tau(x), s(x))
$$


where $e \in G$ is the identity element. By the $G$-equivariance,

$$
\tilde{\tau}(x, h)=(\tau(x), s(x) h)
$$

We have $\tilde{\tau} \circ \tilde{\tau}=\mathrm{id}_{\tilde{\mathrm{P}}}$, so

$$
s(\tau(x))=s(x)^{-1}
$$

Conversely, given any smooth map $s: \tilde{\Sigma} \rightarrow G$ such that (3.4) holds, we define $\tilde{\tau}_{s}: \tilde{P} \rightarrow \tilde{P}$ by $(3.3)$. Then $\tilde{\tau}_{s}$ is a $G$-equivariant involution on $\tilde{P}$ which covers the involution $\tau$ on $\tilde{\Sigma}$, so $P_{s}=\tilde{P} / \tilde{\tau}_{s}$ is a principal $G$-bundle over $\Sigma$.

In particular, we can take $s$ to be a constant map: $s(x) \equiv \epsilon$, where $\epsilon \in G$, $\epsilon^{2}=e$. The involution $\tilde{\tau}_{\epsilon} \equiv \tilde{\tau}_{s}$ on $\tilde{P} \cong \tilde{\Sigma} \times G$ is given by

$$
(x, h) \longmapsto(\tau(x), \epsilon h) .
$$

The zero connection on $\tilde{\Sigma} \times G$ descends to a flat connection $A_{\epsilon}$ on $P_{\epsilon}=\tilde{P} / \tilde{\tau}_{\epsilon}$ which corresponds to

$$
(e, \ldots, e, \epsilon) \in X_{\text {flat }}^{\ell, i}(G) \subset G^{2 \ell+i} .
$$

The topological type of $P_{\epsilon}=\tilde{P} / \tilde{\tau}_{\epsilon}$ can be determined by the following way (see [11]). We use the notation in Section 2.3. Choose $\tilde{\epsilon} \in \rho^{-1}(\epsilon)$, where $\rho: \tilde{G} \rightarrow G$ is the universal covering. The obstruction class

$$
\left[\tilde{\epsilon}^{2}\right] \in \operatorname{Ker} \rho / 2 \operatorname{Ker} \rho \cong \pi_{1}(G) / 2 \pi_{1}(G) \cong H^{2}\left(\Sigma ; \pi_{1}(G)\right)
$$

is independent of the choice of $\tilde{\epsilon}$ and determines the topological type of $P_{\epsilon}$. Recall that $\operatorname{Ker} \rho \cong \pi_{1}(G)$ is abelian, and

$$
\operatorname{Prin}_{G}(\Sigma) \cong H^{2}\left(\Sigma ; \pi_{1}(G)\right) .
$$

Conversely, a principal $G$-bundle over $\Sigma$ of any topological type arises this way. Recall that

$$
\operatorname{Ker} \rho \subset Z(\tilde{G}) \subset \mathfrak{h} \times \tilde{T}_{s s}
$$

where $\tilde{T}_{s s}$ is some maximal torus of $\tilde{G}_{s s}$. Given $k \in \operatorname{Ker} \rho / 2 \operatorname{Ker} \rho$ represented by $a \in \operatorname{Ker} \rho$, choose $\tilde{\epsilon} \in \mathfrak{h} \times \tilde{T}_{s s}$ such that $\tilde{\epsilon}^{2}=a$. Let $\epsilon=\rho(\tilde{\epsilon}) \in G$. Then $\epsilon^{2}=e$, and $\epsilon$ defines a principal $G$-bundle $P_{\epsilon} \rightarrow \Sigma$ with obstruction class $k$.

Example 3.1. $G=U(n)$. Let $\epsilon \in U(n)$ such that $\epsilon^{2}=I_{n}$ (in particular, $\operatorname{det}(\epsilon)= \pm 1)$. Then $c_{1}\left(P_{\epsilon}\right)=c_{1}\left(\operatorname{det}\left(P_{\epsilon}\right)\right)$, where $\operatorname{det}\left(P_{\epsilon}\right)$ is the $U(1)$-bundle 
on $\Sigma$ which is the quotient of $\tilde{\Sigma} \times U(1)$ by the involution

$$
(x, h) \longmapsto(\tau(x), \operatorname{det}(\epsilon) h)
$$

for $x \in \tilde{\Sigma}, h \in U(1)$. So $P_{\epsilon} \cong P_{ \pm}$if $\operatorname{det}(\epsilon)= \pm 1$, where $c_{1}\left(P_{+}\right)=0$ and $c_{1}\left(P_{-}\right)=1$ in $H^{2}(\Sigma ; \mathbb{Z}) \cong \mathbb{Z} / 2 \mathbb{Z}$.

\subsection{Involution on the adjoint bundle}

Let $\mathfrak{g}$ denote the Lie algebra of $G$. Let $\tilde{P}=\tilde{\Sigma} \times G$ be the trivial principal $G$-bundle as above. Let $\operatorname{ad}(\tilde{P})=\tilde{P} \times_{G} \mathfrak{g}$, where $G$ acts on $\tilde{P} \times \mathfrak{g}$ by

$$
g \cdot(x, h, X)=\left(x, h g, \operatorname{Ad}\left(g^{-1}\right)(X)\right)
$$

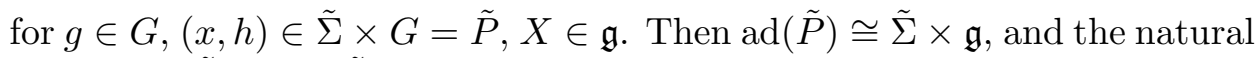
projection $\tilde{P} \times \mathfrak{g} \rightarrow \tilde{\Sigma} \times \mathfrak{g}$ is given by

$$
(x, h, X) \longmapsto(x, \operatorname{Ad}(h)(X)) .
$$

Let $s: \tilde{\Sigma} \rightarrow G$ be a smooth map such that $s(\tau(x))=s(x)^{-1}$, as in (3.4); define $\tilde{\tau}_{s}: \tilde{P} \rightarrow \tilde{P}$ by $\tilde{\tau}_{s}(x, h)=(\tau(x), s(x) h)$, as in (3.3). The involution $\tilde{\tau}_{s}$ on $\tilde{P}$ induces an involution on $\operatorname{ad}(\tilde{P})$ :

$$
(x, X) \longmapsto(\tau(x), \operatorname{Ad}(s(x))(X)) .
$$

We use the same notation $\tilde{\tau}_{s}$ to denote it. We have

$$
\operatorname{ad}(\tilde{P}) / \tilde{\tau}_{s} \cong P_{s} \times_{G} \mathfrak{g}
$$

\subsection{Involution on the space of connections}

$\tilde{\tau}_{s}: \operatorname{ad}(\tilde{P}) \rightarrow \operatorname{ad}(\tilde{P})$ induces an involution

$$
\tilde{\tau}_{s}^{*}: \mathcal{A}(\tilde{P}) \longrightarrow \mathcal{A}(\tilde{P})
$$

where $\mathcal{A}(\tilde{P}) \cong \Omega^{1}(\tilde{\Sigma} ; \mathfrak{g})$. 
More explicitly, given $\theta \in \Omega^{1}(\tilde{\Sigma})$ and $X \in \Omega^{0}(\tilde{\Sigma} ; \mathfrak{g})$,

$$
\tilde{\tau}_{s}^{*}(X \otimes \theta)=\operatorname{Ad}(s)\left(\tau^{*} X\right) \otimes \tau^{*} \theta
$$

Similarly, $\tilde{\tau}_{s}: \operatorname{ad}(\tilde{P}) \rightarrow \operatorname{ad}(\tilde{P})$ induces an involution on $\Omega^{2}(\tilde{\Sigma} ; \mathfrak{g})$. The curvature form $F(A)$ can be viewed as an element in $\Omega^{2}(\tilde{\Sigma} ; \mathfrak{g})$ :

$$
F(A)=X \otimes \omega
$$

where $X: \tilde{\Sigma} \rightarrow \mathfrak{g}$ and $\omega$ is the volume form of $\tilde{\Sigma}$. We have

$F\left(\tilde{\tau}_{s}^{*} A\right)=\tilde{\tau}_{s}^{*}(F(A))=\tilde{\tau}_{s}^{*}(X \otimes \omega)=\operatorname{Ad}(s)\left(\tau^{*} X\right) \otimes \tau^{*} \omega=-\operatorname{Ad}(s)\left(\tau^{*} X\right) \otimes \omega$

where we have used the fact that $\tau$ is anti-symplectic.

Recall that $\mathcal{A}(\tilde{P})$ is a Kähler manifold: the complex structure is given by $\alpha \mapsto * \alpha$, and the symplectic form $\Omega$ is given by

$$
\Omega(\alpha, \beta)=\int_{\tilde{\Sigma}} \operatorname{Tr}(\alpha \wedge \beta) .
$$

The involution $\tilde{\tau}_{s}^{*}: \mathcal{A}(\tilde{P}) \rightarrow \mathcal{A}(\tilde{P})$ is anti-holomorphic and anti-symplectic. The fixed locus $\mathcal{A}(\tilde{P})^{\tilde{\tau}_{s}^{*}}$ can be identified with $\mathcal{A}\left(P_{s}\right)$, the space of $G$ connections on $P_{s}=\tilde{P} / \tilde{\tau}_{s} . \mathcal{A}(\tilde{P})^{\tilde{\tau}_{s}^{*}}$ is a totally geodesic, totally real, Lagrangian submanifold of $\mathcal{A}(\tilde{P})$.

\subsection{Yang-Mills functional and Yang-Mills connections on nonorientable surfaces}

Let $(M, g)$ be a Riemannian manifold with an isometric involution $\tau: M \rightarrow$ $M$. It is straightforward to check the following statements.

Lemma 3.2. Let $f: M \rightarrow \mathbb{R}$ be a smooth function such that $f \circ \tau=f$.

1. Let $N$ be the set of critical points of $f$. Then $\tau(N)=N$.

2. Let $X$ be the gradient vector field of $f$. Then

(a) For any $p \in M$, we have $\tau_{*}(X(p))=X(\tau(p))$.

(b) If $\gamma: I \rightarrow M$ is an integral curve of $X$, where $I$ is an open subset of $\mathbb{R}$, so is $\tau \circ \gamma: I \rightarrow M$. 
Let $M^{\tau}$ be the fixed locus of $\tau$. Suppose that

$$
M^{\tau}=\bigcup_{i \in I} M_{i}^{\tau}
$$

is a union of connected components, where each $M_{i}^{\tau}$ is a submanifold of $M$. Then each $M_{i}^{\tau}$ is a totally geodesic submanifold of $M$ because $\tau$ is an isometry. It is straightforward to check the following statements.

Lemma 3.3. Let $f: M \rightarrow \mathbb{R}$ be a smooth function such that $f \circ \tau=f$, and let $f^{\tau}: M^{\tau} \rightarrow \mathbb{R}$ be the restriction of $f$.Then

1. $X(p) \in T_{p}\left(M^{\tau}\right)$ for any $p \in M^{\tau}$, and $\left.X\right|_{M^{\tau}}$ is the gradient vector field of $f^{\tau}$.

2. The set of critical points of $f^{\tau}: M^{\tau} \rightarrow \mathbb{R}$ is $N^{\tau}=N \cap M^{\tau}$, where $N$ is the set of critical points of $f: M \rightarrow \mathbb{R}$.

In our case, $M=\mathcal{A}(\tilde{P})$ and $f$ is the Yang-Mills functional $L$. We define the Yang-Mills functional on $\mathcal{A}(\tilde{P})^{\tilde{\tau}_{s}^{*}} \cong \mathcal{A}\left(P_{s}\right)$ to be $L^{\tilde{\tau}_{s}^{*}}: \mathcal{A}(\tilde{P})^{\tilde{\tau}_{s}^{*}} \rightarrow \mathbb{R}$. We call the critical points of $L^{\tilde{\tau}_{s}^{*}}$ Yang-Mills connections on $P$. By Lemma 3.2, $A$ is a Yang-Mills connection on $P$ if and only if $\pi^{*} A$ is a Yang-Mills connection on $\tilde{P}$.

It is worth mentioning that our definition of Yang-Mills connections on non-orientable surfaces is different from the one introduced by S. Wang in $[29]$.

\section{Yang-Mills connections and representations}

In this section, we introduce the super central extension of the fundamental group of a surface, and establish a precise correspondence between YangMills connections and representations of super central extension. We introduce representation varieties for Yang-Mills connections on orientable and nonorientable surfaces, and introduce extended moduli spaces for nonorientable surfaces.

\subsection{Super central extension of the fundamental group}

To relate Yang-Mills connections to representations, we need to introduce certain extension of the fundamental group of the surface.

Let $\Sigma$ be a closed, compact, connected surface. Given $a \in \pi_{1}(\Sigma)$, let $\operatorname{deg}(a)=w_{1}\left(T_{\Sigma}\right)[a] \in \mathbb{Z} / 2 \mathbb{Z}$, where $[a]$ is the image of $a$ under the group 
homomorphism $\pi_{1}(\Sigma) \rightarrow H_{1}(\Sigma ; \mathbb{Z})=\pi_{1}(\Sigma) /\left[\pi_{1}(\Sigma), \pi_{1}(\Sigma)\right]$, and $w_{1}\left(T_{\Sigma}\right) \in H^{1}$ $(\Sigma ; \mathbb{Z} / 2 \mathbb{Z})$ is the first Stiefel-Whitney class of the tangent bundle of $\Sigma$. More geometrically, if $\gamma: S^{1} \rightarrow \Sigma$ is a loop representing $a \in \pi_{1}(\Sigma)$, then $\operatorname{deg}(a)=0 \in \mathbb{Z} / 2 \mathbb{Z}$ if the rank 2 real vector bundle $\gamma^{*} T_{\Sigma}$ over $S^{1}$ is orientable (or equivalently, topologically trivial); $\operatorname{deg}(a)=1 \in \mathbb{Z} / 2 \mathbb{Z}$ if $\gamma^{*} T_{\Sigma}$ is non-orientable (or equivalently, topologically non-trivial). The group homomorphism deg: $\pi_{1}(\Sigma) \rightarrow \mathbb{Z} / 2 \mathbb{Z}$ is trivial if and only if $\Sigma$ is orientable.

We are now ready to define the super central extension $\Gamma_{\mathbb{R}}(\Sigma)$ of $\pi_{1}(\Sigma)$. It fits in a short exact sequence of groups:

$$
1 \rightarrow \mathbb{R} \stackrel{\alpha}{\longrightarrow} \Gamma_{\mathbb{R}}(\Sigma) \stackrel{\beta}{\longrightarrow} \pi_{1}(\Sigma) \rightarrow 1 .
$$

Given $r \in \mathbb{R}$, let $J_{r}=\alpha(r)$, so that $J_{r_{1}+r_{2}}=J_{r_{1}} J_{r_{2}}$. Given $a \in \Gamma_{\mathbb{R}}(\Sigma)$, we have

$$
a J_{r} a^{-1}=\left\{\begin{array}{l}
J_{r} \\
J_{-r}=J_{r}^{-1}
\end{array} \quad \text { if } \operatorname{deg}(\beta(a))= \begin{cases}0 & \\
1 & \in \mathbb{Z} / 2 \mathbb{Z} .\end{cases}\right.
$$

This defines $\Gamma_{\mathbb{R}}(\Sigma)$ up to group isomorphism. We will give a more explicit description later.

When $\Sigma$ is orientable, $\Gamma_{\mathbb{R}}(\Sigma)$ is the central extension of $\pi_{1}(\Sigma)$ defined in $[1]$.

\subsection{Representation varieties for orientable surfaces}

Recall that any closed, compact, connected surface is diffeomorphic to $\Sigma_{0}^{\ell}$, a Riemann surface of genus $\ell$, for some nonnegative integer $\ell . \Gamma_{\mathbb{R}}\left(\Sigma_{0}^{\ell}\right)$ is generated by

$$
A_{1}, B_{1}, \ldots, A_{\ell}, B_{\ell}, J_{r}
$$

where $r \in \mathbb{R}$, with relations

(1) $J_{r_{1}} J_{r_{2}}=J_{r_{1}+r_{2}}$;

(2) $\left[A_{i}, J_{r}\right]=\left[B_{i}, J_{r}\right]=1, \quad i=1, \ldots, \ell, r \in \mathbb{R}$;

(3) $\prod_{i=1}^{\ell}\left[A_{i}, B_{i}\right]=J_{1}$.

Let $\rho: \Gamma_{\mathbb{R}}\left(\Sigma_{0}^{\ell}\right) \rightarrow G$ be a group homomorphism. From the relation (1), we must have $\rho\left(J_{r}\right)=\exp (r X)$ for some $X \in \mathfrak{g}$, where $\mathfrak{g}$ is the Lie algebra of $G$. From relation (2) we must have $\rho\left(A_{i}\right), \rho\left(B_{i}\right) \in G_{X}$, where $G_{X}$ is the stabilizer of $X$ of the adjoint action of $G$ on $\mathfrak{g}$. Combined with relation (3), 
$\operatorname{Hom}\left(\Gamma_{\mathbb{R}}\left(\Sigma_{0}^{\ell}\right), G\right)$ can be identified with

$$
X_{\mathrm{YM}}^{\ell, 0}(G)=\left\{(V, X) \in G^{2 \ell} \times \mathfrak{g} \mid V \in\left(G_{X}\right)^{2 \ell}, \mathfrak{m}(V)=\exp (X)\right\}
$$

where $\mathfrak{m}\left(a_{1}, b_{1}, \ldots, a_{\ell}, b_{\ell}\right)=\prod_{i=1}^{\ell}\left[a_{i}, b_{i}\right]$ was defined in Section 2.1.

Let $\mathcal{N}(P) \subset \mathcal{A}(P)$ be the space of Yang-Mills connections on $P$. By Theorem 6.16 in $[1], \mathcal{N}(P)$ is nonempty for any underlying principal $G$ bundle $P$. Let $\mathcal{N}_{0}(P) \subset \mathcal{A}(P)$ be the space of flat connections on $P$, as in Section 2. The natural inclusion $\mathcal{N}_{0}(P) \subset \mathcal{N}(P)$ induces an inclusion $X_{\text {flat }}^{\ell, 0}(G) \hookrightarrow X_{\mathrm{YM}}^{\ell, 0}(G), V \mapsto(V, 0)$.

Let $\mathcal{G}(P)$ be the gauge group, and let $\mathcal{G}_{0}(P)$ be the based gauge group, as before.

Theorem 4.1 [1, Theorem 6.7]. There is a bijective correspondence between conjugacy classes of homomorphisms $\Gamma_{\mathbb{R}}(\Sigma)$ and gauge equivalence classes of Yang-Mills G-connections over $\Sigma$. In other words, we have homeomorphisms

$$
\begin{aligned}
& \bigcup_{P \in \operatorname{Prin}_{G}\left(\Sigma_{0}^{\ell}\right)} \mathcal{N}(P) / \mathcal{G}_{0}(P) \cong \operatorname{Hom}\left(\Gamma_{\mathbb{R}}\left(\Sigma_{0}^{\ell}\right), G\right) \cong X_{\mathrm{YM}}^{\ell, 0}(G), \\
& \bigcup_{P \in \operatorname{Prin}_{G}\left(\Sigma_{0}^{\ell}\right)} \mathcal{N}(P) / \mathcal{G}(P) \cong \operatorname{Hom}\left(\Gamma_{\mathbb{R}}\left(\Sigma_{0}^{\ell}\right), G\right) / G \cong X_{\mathrm{YM}}^{\ell, 0}(G) / G,
\end{aligned}
$$

where $g \in G$ acts on $G^{2 \ell} \times \mathfrak{g}$ by $g \cdot(V, X)=\left(g V g^{-1}, \operatorname{Ad}(g)(X)\right)$.

\subsection{Holonomy on the double cover}

Let $\Sigma$ be a closed, compact, connected, nonorientable surface, and let $\pi$ : $\tilde{\Sigma} \rightarrow \Sigma$ be the orientable double cover. Then $\tilde{\Sigma}$ is a closed, compact, connected orientable surface. Let $\tau: \tilde{\Sigma} \rightarrow \tilde{\Sigma}$ be the deck transformation which is an orientation reversing involution.

Let $A \in \mathcal{A}(\tilde{P})$ be a Yang-Mills connection. Recall that $A \in \mathcal{A}(\tilde{P})$ is a Yang-Mills connection if and only if there exists $u: \tilde{\Sigma} \rightarrow G$ such that

$$
F(A)=\operatorname{Ad}(u)(X) \otimes \omega
$$

where $X$ is a constant vector in $\mathfrak{g}$, or equivalently, if there exists $A^{\prime} \in \mathcal{A}(\tilde{P})$ and $u \in \mathcal{G}(\tilde{P})$ such that $A=u \cdot A^{\prime}$ and $F\left(A^{\prime}\right)=X \otimes \omega$. 
We fix a trivialization $\tilde{P} \rightarrow \tilde{\Sigma} \times G$ such that

$$
F(A)=X \otimes \omega
$$

where $X \in \mathfrak{g}$ and $\omega$ is the volume form. Using this trivialization, we may define the holonomy along a path (holonomies along based loops are defined without using the trivialization of $\tilde{P})$. Given a path $\gamma:[0,1] \rightarrow \tilde{\Sigma}$, let $\tilde{\gamma}:[0,1] \rightarrow \tilde{P}$ be the horizontal lifting of $\gamma$ (with respect to the connection $A)$ with $\tilde{\gamma}(0)=(\gamma(0), e)$, where $e$ is the identity element. Then $\tilde{\gamma}(1)=$ $\left(\gamma(1), g^{-1}\right)$ for some $g \in G_{X}$, where $G_{X}$ is the stabilizer of $X \in \mathfrak{g}$ of the adjoint action of $G$ on $\mathfrak{g}$. We call $g \in G$ the holonomy along $\gamma$.

Let $\tilde{\gamma}^{\prime}:[0,1] \rightarrow \tilde{P}$ be another horizontal lifting of $\gamma$ with $\tilde{\gamma}^{\prime}(0)=(\gamma(0), h)$, where $h \in G$. By $G$-invariance of the connection, we have $\tilde{\gamma}^{\prime}=\tilde{\gamma} \cdot h$, so

$$
\tilde{\gamma}^{\prime}(1)=\left(\gamma(1), g^{-1} h\right)=\left(\gamma(1), h\left(h^{-1} g h\right)^{-1}\right)
$$

To summarize, if we change the trivialization by a constant gauge transformation $h$, the curvature form changes from $F(A)=X \otimes \omega \in \Omega^{2}(\tilde{\Sigma} ; \mathfrak{g})$ to

$$
F(A)=\operatorname{Ad}\left(h^{-1}\right) X \otimes \omega \in \Omega^{2}(\tilde{\Sigma} ; \mathfrak{g})
$$

and the holonomy along $\gamma$ changes from $g \in G_{X}$ to $h^{-1} g h \in G_{\operatorname{Ad}\left(h^{-1}\right) X}$.

Recall that $\Sigma$ is diffeomorphic to $\Sigma_{i}^{\ell}$ for some $\ell \geq 0$ and $i=1,2$, where $\Sigma_{1}^{\ell}$ is the connected sum of a Riemann surface of genus $\ell$ and the real projective plane, and $\Sigma_{2}^{\ell}$ is the connected sum of a Riemann surface of genus $\ell$ and a Klein bottle. We will discuss the case $\Sigma_{1}^{\ell}$ in detail. The case $\Sigma_{2}^{\ell}$ is similar.

Suppose that $s: \tilde{\Sigma} \rightarrow G$ satisfies (3.4), so it defines an involution $\tilde{\tau}_{s}$ : $\tilde{P} \rightarrow \tilde{P}$. Now look at Figure 1 .

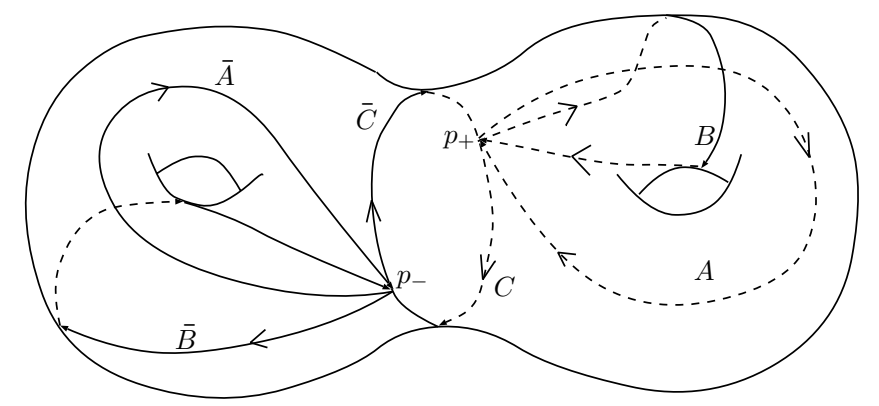

Figure 1: Holonomy on the double cover $\Sigma_{0}^{2}$. 
$A_{i}, B_{i}$ are loops passing through $p_{+} \in \tilde{\Sigma}, \bar{A}_{i}, \bar{B}_{i}$ are loops passing through $p_{-}=\tau\left(p_{+}\right), C$ is a path from $p_{+}$to $p_{-}$, and $\bar{C}$ is a path from $p_{-}$to $p_{+}$. The holonomies along $A_{i}, B_{i}, \bar{A}_{i}, \bar{B}_{i}$ depend on the connection, not on the trivialization. The holonomies along $C$ and $\bar{C}$ depend on the connection and the trivialization. We choose the trivialization as follows. Let the trivialization of $\tilde{P}$ at $p_{+}$and $p_{-}$be given by $h \mapsto h$ and $h \mapsto s\left(p_{+}\right) h$, respectively. We define $c$ and $\bar{c}$ as follows. Let $\gamma:[0,1] \rightarrow \tilde{P}$ be the horizontal lifting of $C$ such that $\gamma(0)=\left(p_{+}, e\right)$. Then $\gamma(1)=\left(p_{-}, s\left(p_{+}\right) c^{-1}\right)$. Let $\bar{\gamma}:[0,1] \rightarrow \tilde{P}$ be the horizontal lifting of $\bar{C}$ such that $\bar{\gamma}(0)=\left(p_{-}, s\left(p_{+}\right)\right)$. Then $\bar{\gamma}(1)=\left(p_{+}, \bar{c}^{-1}\right)$. Let $\gamma^{\prime}(t)=\bar{\gamma}(t) \cdot c^{-1}$. Then $\gamma^{\prime}$ is also a horizontal lifting of $\bar{C}, \gamma^{\prime}(0)=\left(p_{-}, s\left(p_{+}\right) c^{-1}\right), \gamma^{\prime}(1)=\left(p_{+}, \bar{c}^{-1} c^{-1}\right)=\left(p_{+},(c \bar{c})^{-1}\right)$. So $\gamma \cup \gamma^{\prime}$ is a horizontal lifting of $C \bar{C}$, and the holonomy along $C \bar{C}$ is $c \bar{c}$. Denote the holonomies along $A_{i}, B_{i}, \bar{A}_{i}, \bar{B}_{i}$ by $a_{i}, b_{i}, \bar{a}_{i}, \bar{b}_{i}$, respectively.

We cut $\tilde{\Sigma}$ into two discs $D_{+}$and $D_{-}=\tau\left(D_{+}\right)$. The (oriented) boundaries of $D_{+}$and $D_{-}$are

$$
\partial D_{+}=\prod_{i=1}^{\ell}\left[\bar{A}_{i}, \bar{B}_{i}\right] C^{-1} \bar{C}^{-1}, \quad \partial D_{-}=\prod_{i=1}^{\ell}\left[A_{i}, B_{i}\right] \bar{C}^{-1} C^{-1}
$$

Recall that

$$
\int_{\tilde{\Sigma}} \omega=1, \quad \int_{\tilde{\Sigma}} \omega=\int_{D_{+}} \omega+\int_{-D_{-}} \omega, \quad \tau^{*} \omega=-\omega
$$

where $-D_{-}$is $D_{-}$with the reversed orientation. We conclude that

$$
\int_{D_{ \pm}} \omega= \pm \frac{1}{2}
$$

Let $z=s\left(p_{+}\right) \in G$. From the above discussion, we have

$$
\begin{aligned}
\mathfrak{m}(V) \bar{c}^{-1} c^{-1} & =\exp \left(-\int_{D_{-}} X \otimes \omega\right)=\exp (X / 2) \\
\mathfrak{m}(\bar{V}) c^{-1} \bar{c}^{-1} & =\exp \left(-\int_{D_{+}} \operatorname{Ad}\left(z^{-1}\right) X \otimes \omega\right)=\exp \left(-\operatorname{Ad}\left(z^{-1}\right) X / 2\right)
\end{aligned}
$$

Moreover,

$$
V \in\left(G_{X}\right)^{2 \ell}, \quad c z^{-1} \in G_{X}, \quad \bar{V} \in\left(G_{\operatorname{Ad}\left(z^{-1}\right)(X)}\right)^{2 \ell}, \quad \bar{c} z \in G_{\operatorname{Ad}\left(z^{-1}\right)(X)} .
$$


So we shall define a symmetric representation variety

$$
\begin{aligned}
Z_{\mathrm{YM}}^{\ell, 1}(G)^{z}= & \left\{(V, c, \bar{V}, \bar{c}, X) \in G^{2(2 \ell+1)} \times \mathfrak{g} \mid V \in\left(G_{X}\right)^{2 \ell}, c z^{-1} \in G_{X},\right. \\
& \bar{V} \in\left(G_{\operatorname{Ad}\left(z^{-1}(X)\right.}\right)^{2 \ell}, \bar{c} z \in G_{\operatorname{Ad}\left(z^{-1}\right)(X)}, \mathfrak{m}(V)=\exp (X / 2) c \bar{c} \\
& \left.\mathfrak{m}(\bar{V})=\exp \left(-\operatorname{Ad}\left(z^{-1}\right) X / 2\right) \bar{c} c\right\}
\end{aligned}
$$

Our next goal is to rewrite $Z_{\mathrm{YM}}^{\ell, 1}(G)^{z}$ without using $z$. Given $(V, c, \bar{V}, \bar{c}$, $X) \in Z_{\mathrm{YM}}^{\ell, 1}(G)^{z}$, we have $\bar{c} z \in G_{\operatorname{Ad}\left(z^{-1}\right)(X)}$, which implies $\operatorname{Ad}\left(z^{-1}\right)(X)=$ $\operatorname{Ad}(\bar{c})(X)$. So

$$
\mathfrak{m}(\bar{V})=\exp (-\operatorname{Ad}(\bar{c})(X) / 2) \bar{c} c=\bar{c} \exp (-X / 2) c
$$

We also have

$$
c \bar{c} \in G_{X}, \quad \bar{V} \in\left(G_{\operatorname{Ad}(\bar{c})(X)}\right)^{2 \ell}, \quad \bar{c} c \in G_{\operatorname{Ad}(\bar{c})(X)},
$$

which imply

$$
c \bar{V} c^{-1} \in\left(G_{X}\right)^{2 \ell}
$$

Define

$$
\begin{aligned}
& Z_{\mathrm{YM}}^{\ell, 1}(G)=\left\{(V, c, \bar{V}, \bar{c}, X) \in G^{2(2 \ell+1)} \times \mathfrak{g} \mid V, c \bar{V} c^{-1} \in\left(G_{X}\right)^{2 \ell}\right. \\
&\mathfrak{m}(V)=\exp (X / 2) c \bar{c}, \mathfrak{m}(\bar{V})=\bar{c} \exp (-X / 2) c\}
\end{aligned}
$$

Then

$$
Z_{\mathrm{YM}}^{\ell, 1}(G)^{z}=\left\{(V, c, \bar{V}, \bar{c}, X) \in Z_{\mathrm{YM}}^{\ell, 1}(G) \mid \operatorname{Ad}(\bar{c})(X)=\operatorname{Ad}\left(z^{-1}\right)(X)\right\},
$$

where $V, \bar{V} \in G^{2 \ell}, c, \bar{c} \in G, X \in \mathfrak{g}$, and

$$
Z_{\mathrm{YM}}^{\ell, 1}(G)=\bigcup_{z \in G} Z_{\mathrm{YM}}^{\ell, 1}(G)^{z} .
$$

The involution $\tilde{\tau}_{s}: \mathcal{A}(\tilde{P}) \rightarrow \mathcal{A}(\tilde{P})$ induces a map $\tau_{z}: Z_{\mathrm{YM}}^{\ell, 1}(G)^{z} \rightarrow$ $Z_{\mathrm{YM}}^{\ell, 1}(G)^{z^{-1}}$ given by

$$
(V, c, \bar{V}, \bar{c}, X) \longmapsto\left(\bar{V}, \bar{c}, V, c,-\operatorname{Ad}\left(z^{-1}\right) X\right),
$$

or equivalently,

$$
(V, c, \bar{V}, \bar{c}, X) \longmapsto(\bar{V}, \bar{c}, V, c,-\operatorname{Ad}(\bar{c}) X) .
$$


Note that (4.1) defines an involution $\tau: Z_{\mathrm{YM}}^{\ell, 1}(G) \rightarrow Z_{\mathrm{YM}}^{\ell, 1}(G)$. Similarly, the involution $\tilde{\tau}_{s}^{*}: \mathcal{A}(\tilde{P}) \rightarrow \mathcal{A}(\tilde{P})$ induces an involution $\tau: Z_{\mathrm{YM}}^{\ell, 2}(G) \rightarrow Z_{\mathrm{YM}}^{\ell, 2}(G)$ given by

$$
(V, d, c, \bar{V}, \bar{d}, \bar{c}, X) \longmapsto(\bar{V}, \bar{d}, \bar{c}, V, d, c,-\operatorname{Ad}(\bar{c}) X)
$$

\subsection{Symmetric representation varieties}

In this subsection, $i=1,2$. Based on the discussion in Section 4.3, we define symmetric representation varieties as follows:

$$
\begin{gathered}
Z_{\mathrm{YM}}^{\ell, 1}(G)=\left\{(V, c, \bar{V}, \bar{c}, X) \in G^{2(2 \ell+1)} \times \mathfrak{g} \mid V, c \bar{V} c^{-1} \in\left(G_{X}\right)^{2 \ell}\right. \\
\mathfrak{m}(V)=\exp (X / 2) c \bar{c}, \mathfrak{m}(\bar{V})=\bar{c} \exp (-X / 2) c\} \\
Z_{\mathrm{YM}}^{\ell, 2}(G)=\left\{(V, d, c, \bar{V}, \bar{d}, \bar{c}, X) \in G^{2(2 \ell+2)} \times \mathfrak{g} \mid V, d^{-1} c \bar{V} c^{-1} d \in\left(G_{X}\right)^{2 \ell},\right. \\
d^{-1}, c \bar{c} \in G_{X}, \quad \mathfrak{m}(V)=\exp (X / 2) c \bar{d} c^{-1} d \\
\left.\mathfrak{m}(\bar{V})=\bar{c} d \exp (-X / 2) \bar{c}^{-1} \bar{d}\right\}
\end{gathered}
$$

where $\mathfrak{m}\left(a_{1}, b_{1}, \ldots, a_{\ell}, b_{\ell}\right)=\prod_{i=1}^{\ell}\left[a_{i}, b_{i}\right]$ is defined as in Section 2.1.

Lemma 4.2. For $i=1,2$, define $\Phi_{G}^{\ell, i}: G^{2(2 \ell+i)} \times \mathfrak{g} \rightarrow G^{2(2 \ell+i-1)} \times \mathfrak{g}$ by

$$
\begin{aligned}
\Phi_{G}^{\ell, 1}(V, c, \bar{V}, \bar{c}, X) & =\left(V, c \mathfrak{r}(\bar{V}) c^{-1}, X\right), \\
\Phi_{G}^{\ell, 2}(V, d, c, \bar{V}, \bar{d}, \bar{c}, X) & =\left(V, d^{-1} c \mathfrak{r}(\bar{V}) c^{-1} d, d^{-1}, c \bar{c}, X\right),
\end{aligned}
$$

where $V, \bar{V} \in G^{2 \ell}, \quad c, d \in G, \quad X \in \mathfrak{g}, \quad$ and $\quad \mathfrak{r}\left(a_{1}, b_{1}, \ldots, a_{\ell}, b_{\ell}\right)=\left(b_{\ell}, a_{\ell}\right.$, $\left.\ldots, b_{1}, a_{1}\right)$. Then

$$
\Phi_{G}^{\ell, i}\left(Z_{\mathrm{YM}}^{\ell, i}(G)\right)=X_{\mathrm{YM}}^{2 \ell+i-1,0}(G) .
$$

There are inclusions $Z_{\text {flat }}^{\ell, i}(G) \hookrightarrow Z_{\mathrm{YM}}^{\ell, i}(G)$ given by $(V, v, \bar{V}, \bar{v}) \mapsto$ $(V, v, \bar{V}, \bar{v}, 0)$, where $V, \bar{V} \in G^{2 \ell}$, and $v, \bar{v} \in G^{i}$. We use the same notation for $\Phi_{G}^{\ell, i}$ in Lemma 2.3 and Lemma 4.2 , since $\Phi_{G}^{\ell, i}$ in Lemma 2.3 is just the restriction of $\Phi_{G}^{\ell, i}$ in Lemma 4.2 .

Proof of Lemma 4.2. $\quad$ 1. Claim: $\Phi_{G}^{\ell, 1}\left(Z_{\mathrm{YM}}^{\ell, 1}(G)\right) \subset X_{\mathrm{YM}}^{2 \ell, 0}(G)$. 
Given $(V, c, \bar{V}, \bar{c}, X) \in Z_{\mathrm{YM}}^{\ell, 1}(G)$, where $V, \bar{V} \in G^{2 \ell}, c, \bar{c} \in G$, and $X \in$ $\mathfrak{g}$, we have

$V, c \bar{V} c^{-1} \in\left(G_{X}\right)^{2 \ell}, \quad \mathfrak{m}(V)=\exp (X / 2) c \bar{c}, \quad \mathfrak{m}(\bar{V})=\bar{c} \exp (-X / 2) c$

Straightforward calculations show that $c \bar{c}=\exp (-X / 2) \mathfrak{m}(V) \in G_{X}$, and

$$
\mathfrak{m}\left(c \mathfrak{r}(\bar{V}) c^{-1}\right)=c \mathfrak{m}(\mathfrak{r}(\bar{V})) c^{-1}=\exp (X / 2)(c \bar{c})^{-1}
$$

So if $(V, c, \bar{V}, \bar{c}, X) \in Z_{\mathrm{YM}}^{\ell, 1}(G)$, then

$$
\begin{aligned}
\mathfrak{m}(V) \mathfrak{m}\left(c \mathfrak{r}(\bar{V}) c^{-1}\right) & =\exp (X / 2) c \bar{c} \exp (X / 2)(c \bar{c})^{-1} \\
& =\exp (X / 2) \exp (\operatorname{Ad}(c \bar{c})(X) / 2)=\exp (X)
\end{aligned}
$$

where we have used $c \bar{c} \in G_{X}$, i.e., $\operatorname{Ad}(c \bar{c})(X)=X$. In other words,

$$
\Phi_{G}^{\ell, 1}(V, c, \bar{V}, \bar{c}, X)=\left(V, c \mathfrak{r}(\bar{V}) c^{-1}, X\right) \in X_{\mathrm{YM}}^{2 \ell, 0}(G)
$$

2. Claim: $X_{\mathrm{YM}}^{2 \ell, 0}(G) \subset \Phi_{G}^{\ell, 1}\left(Z_{\mathrm{YM}}^{\ell, 1}(G)\right)$. have

Given $\left(V_{1}, V_{2}, X\right) \in X_{\mathrm{YM}}^{2 \ell, 0}(G)$, where $V_{1}, V_{2} \in G^{2 \ell}$ and $X \in \mathfrak{g}$, we

$$
V_{1}, V_{2} \in\left(G_{X}\right)^{2 \ell}, \quad \mathfrak{m}\left(V_{1}\right) \mathfrak{m}\left(V_{2}\right)=\exp (X)
$$

Let $\bar{c}=\exp (-X / 2) \mathfrak{m}\left(V_{1}\right)$. Then $\bar{c} \in G_{X}$, and

$$
\bar{c}=\exp (-X / 2) \exp (X) \mathfrak{m}\left(V_{2}\right)^{-1}=\exp (X / 2) \mathfrak{m}\left(\mathfrak{r}\left(V_{2}\right)\right) .
$$

We have

$$
\mathfrak{m}\left(V_{1}\right)=\exp (X / 2) \bar{c}, \quad \mathfrak{m}\left(\mathfrak{r}\left(V_{2}\right)\right)=\exp (-X / 2) \bar{c}=\bar{c} \exp (-X / 2)
$$

So

$$
\left(V_{1}, e, \mathfrak{r}\left(V_{2}\right), \bar{c}, X\right) \in Z_{\mathrm{YM}}^{\ell, 1}(G)
$$

and

$$
\left(V_{1}, V_{2}, X\right)=\Phi_{G}^{\ell, 1}\left(V_{1}, e, \mathfrak{r}\left(V_{2}\right), \bar{c}, X\right) \in \Phi_{G}^{\ell, 1}\left(Z_{\mathrm{YM}}^{\ell, 1}(G)\right)
$$

3. Claim: $\Phi_{G}^{\ell, 2}\left(Z_{\mathrm{YM}}^{\ell, 2}(G)\right) \subset X_{\mathrm{YM}}^{2 \ell+1,0}(G)$. 
Given $(V, d, c, \bar{V}, \bar{d}, \bar{c}, X) \in Z_{\mathrm{YM}}^{\ell, 2}(G)$, where $V, \bar{V} \in G^{2 \ell}, d, c, \bar{d}, \bar{c} \in G$, and $X \in \mathfrak{g}$, we have

$$
\begin{aligned}
V, d^{-1} c \mathfrak{r}(\bar{V}) c^{-1} d \in\left(G_{X}\right)^{2 \ell}, \mathfrak{m}(V) & =\exp (X / 2) c \bar{d} c^{-1} d, \mathfrak{m}(\bar{V}) \\
& =\bar{c} d \exp (-X / 2) \bar{c}^{-1} \bar{d}
\end{aligned}
$$

Straightforward computations show that

$$
\begin{aligned}
\mathfrak{m}(V) \mathfrak{m}\left(d^{-1} c \mathfrak{r}(\bar{V}) c^{-1} d\right)\left[d^{-1}, c \bar{c}\right] & =\mathfrak{m}(V) d^{-1} c \mathfrak{m}(\bar{V})^{-1} c^{-1} d\left[d^{-1}, c \bar{c}\right] \\
& =\exp (X / 2) \exp (\operatorname{Ad}(c \bar{c})(X) / 2) \\
& =\exp (X)
\end{aligned}
$$

where we also used $c \bar{c} \in G_{X}$, i.e., $\operatorname{Ad}(c \bar{c})(X)=X$. In other words,

$$
\Phi_{G}^{\ell, 2}(V, d, c, \bar{V}, \bar{d}, \bar{c}, X)=\left(V, d^{-1} c \mathfrak{r}(\bar{V}) c^{-1} d, d^{-1}, c \bar{c}, X\right) \in X_{\mathrm{YM}}^{2 \ell+1,0}(G) .
$$

4. Claim: $X_{\mathrm{YM}}^{2 \ell+1,0}(G) \subset \Phi_{G}^{\ell, 2}\left(Z_{\mathrm{YM}}^{\ell, 2}(G)\right)$.

Given $\left(V_{1}, V_{2}, a, b, X\right) \in X_{\mathrm{YM}}^{2 \ell+1,0}(G)$, where $V_{1}, V_{2} \in G^{2 \ell}$ and $a, b \in$ $G$, we have

$$
V_{1}, V_{2} \in\left(G_{X}\right)^{2 \ell}, \quad a, b \in G_{X}, \quad \mathfrak{m}\left(V_{1}\right) \mathfrak{m}\left(V_{2}\right)[a, b]=\exp (X) .
$$

Let

$$
d=a^{-1}, \quad \bar{d}=a \exp (-X / 2) \mathfrak{m}\left(V_{1}\right), \quad c=a^{-1}, \quad \bar{c}=a b .
$$

Then

$$
\exp (X / 2) c \bar{d} c^{-1} d=\mathfrak{m}\left(V_{1}\right)
$$

and

$$
\begin{aligned}
\bar{c} d \exp (-X / 2) \bar{c}^{-1} \bar{d}= & a b a^{-1} \exp (-X / 2) b^{-1} \exp (-X / 2) \mathfrak{m}\left(V_{1}\right) \\
= & {[a, b] \exp (-\operatorname{Ad}(b)(X) / 2) \exp (-X / 2) } \\
& \times \exp (X)\left(\mathfrak{m}\left(V_{2}\right)[a, b]\right)^{-1}=\mathfrak{m}\left(\mathfrak{r}\left(V_{2}\right)\right)
\end{aligned}
$$

where we have used that $b \in G_{X}$ (i.e. $\left.\operatorname{Ad}(b)(X)=X\right)$ in the last equality. So

$$
\left(V_{1}, d, c, \mathfrak{r}\left(V_{2}\right), \bar{d}, \bar{c}, X\right) \in Z_{\mathrm{YM}}^{\ell, 2}(G),
$$

and

$$
\left(V_{1}, V_{2}, a, b, X\right)=\Phi_{G}^{\ell, 2}\left(V_{1}, d, c, \mathfrak{r}\left(V_{2}\right), \bar{d}, \bar{c}, X\right)
$$


Let $G^{2}$ act on $Z_{\mathrm{YM}}^{\ell, 1}(G), Z_{\mathrm{YM}}^{\ell, 2}(G)$ by

$$
\begin{aligned}
\left(g_{1}, g_{2}\right) \cdot(V, c, \bar{V}, \bar{c}, X)= & \left(g_{1} V g_{1}^{-1}, g_{1} c g_{2}^{-1}, g_{2} \bar{V} g_{2}^{-1}, g_{2} \bar{c} g_{1}^{-1}, \operatorname{Ad}\left(g_{1}\right)(X)\right), \\
\left(g_{1}, g_{2}\right) \cdot(V, d, c, \bar{V}, \bar{d}, \bar{c}, X)= & \left(g_{1} V g_{1}^{-1}, g_{1} d g_{1}^{-1}, g_{1} c g_{2}^{-1}, g_{2} V g_{2}^{-1}, g_{2}\right. \\
& \left.\times \bar{d} g_{2}^{-1}, g_{2} \bar{c} g_{1}^{-1}, \operatorname{Ad}\left(g_{1}\right)(X)\right)
\end{aligned}
$$

respectively, where $V, \bar{V} \in G^{2 \ell}$, and $g_{1}, g_{2}, c, \bar{c}, d, \bar{d} \in G$. Slight modification of the proof of Lemma 2.4 gives the following:

Lemma 4.3. The surjection $\Phi_{G}^{\ell, i}: Z_{\mathrm{YM}}^{\ell, i}(G) \rightarrow X_{\mathrm{YM}}^{2 \ell+i-1,0}(G)$ induces homeomorphisms

$$
Z_{\mathrm{YM}}^{\ell, i}(G) / G^{2} \cong X_{\mathrm{YM}}^{2 \ell+i-1,0}(G) / G \cong \operatorname{Hom}\left(\Gamma_{\mathbb{R}}\left(\Sigma_{0}^{2 \ell+i-1}\right), G\right) / G
$$

and a homotopy equivalence

$$
Z_{\mathrm{YM}}^{\ell, i}(G)^{h G^{2}} \sim X_{\mathrm{YM}}^{2 \ell+i-1,0}(G)^{h G}
$$

between homotopic orbit spaces.

\subsection{Involution on representation varieties for Yang-Mills connections}

Lemma 4.4. For $i=1,2$, define $\tau: G^{2(2 \ell+i)} \times \mathfrak{g} \rightarrow G^{2(2 \ell+i)} \times \mathfrak{g}$ by

$$
\begin{aligned}
\tau(V, c, \bar{V}, \bar{c}, X) & =(\bar{V}, \bar{c}, V, c,-\operatorname{Ad}(\bar{c}) X), \\
\tau(V, d, c, \bar{V}, \bar{d}, \bar{c}, X) & =(\bar{V}, \bar{d}, \bar{c}, V, d, c,-\operatorname{Ad}(\bar{c}) X),
\end{aligned}
$$

where $V \in G^{2 \ell}, c, \bar{c}, d, \bar{d} \in G, X \in \mathfrak{g}$. Then

$$
\tau\left(Z_{\mathrm{YM}}^{\ell, i}(G)\right)=Z_{\mathrm{YM}}^{\ell, i}(G)
$$

and $\tau \circ \tau$ restricts to the identity map on $Z_{\mathrm{YM}}^{\ell, i}(G)$.

Remark 4.5. Based on (4.1) and (4.2), the involution $\tau$ defined in Lemma 4.4 is the one induced by the $\mathbb{Z} / 2 \mathbb{Z}$ deck transformation on the double cover. 
Proof of Lemma 4.4. We first prove $\tau\left(Z_{\mathrm{YM}}^{\ell, i}(G)\right) \subset Z_{\mathrm{YM}}^{\ell, i}(G) . \quad i=1$ : Given $(V, c, \bar{V}, \bar{c}, X) \in Z_{\mathrm{YM}}^{\ell, 1}(G)$, where $V, \bar{V} \in G^{2 \ell}, c, \bar{c} \in G$, and $X \in \mathfrak{g}$, we have

$$
V, c \bar{V} c^{-1} \in\left(G_{X}\right)^{2 \ell}, \quad c \bar{c} \in G_{X},
$$

so $\bar{c}^{-1} \bar{V} \bar{c}=(c \bar{c})^{-1}\left(c \bar{V} c^{-1}\right)(c \bar{c}) \in\left(G_{X}\right)^{2 \ell}$, or equivalently,

(i) $\bar{V} \in\left(\bar{c} G_{X} \bar{c}^{-1}\right)^{2 \ell}=\left(G_{\operatorname{Ad}(\bar{c})(X)}\right)^{2 \ell}=\left(G_{-\operatorname{Ad}(\bar{c})(X)}\right)^{2 \ell}$.

If we let $\bar{X}=-\operatorname{Ad}(\bar{c})(X)$, then we have $\bar{V} \in\left(G_{\bar{X}}\right)^{2 \ell}$. We also have

(ii) $\bar{c} V \bar{c}^{-1} \in\left(\bar{c} G_{X} \bar{c}^{-1}\right)^{2 \ell}=\left(G_{\operatorname{Ad}(\bar{c})(X)}\right)^{2 \ell}=\left(G_{\bar{X}}\right)^{2 \ell}$.

To summarize, we have

$$
\bar{V}, \bar{c} V \bar{c}^{-1} \in\left(G_{\bar{X}}\right)^{2 \ell}
$$

We also have

$$
\begin{aligned}
\mathfrak{m}(\bar{V}) & =\bar{c} \exp (-X / 2) c=\left(\bar{c} \exp (-X / 2) \bar{c}^{-1}\right) \bar{c} c \\
& =\exp (\bar{X} / 2) \bar{c} c \\
\mathfrak{m}(V) & =\exp (X / 2) c \bar{c}=c \bar{c} \exp (X / 2)=c\left(\bar{c} \exp (X / 2) \bar{c}^{-1}\right) \bar{c} \\
& =c \exp (-\bar{X} / 2) \bar{c} .
\end{aligned}
$$

By $(4.5)-(4.7)$, we get $\tau(V, c, \bar{V}, \bar{c}, X)=(\bar{V}, \bar{c}, V, c, \bar{X}) \in Z_{\mathrm{YM}}^{\ell, 1}(G)$. This proves

$$
\tau\left(Z_{\mathrm{YM}}^{\ell, 1}(G)\right) \subset Z_{\mathrm{YM}}^{\ell, 1}(G) .
$$

$i=2$ : Given $(V, d, c, \bar{V}, \bar{d}, \bar{c}, X) \in Z_{\mathrm{YM}}^{\ell, 2}(G)$, where $V, \bar{V} \in G^{2 \ell}, d, c, \bar{d}, \bar{c} \in G$, and $\mathfrak{g} \in X$, we have

$$
V, d^{-1} c \bar{V} c^{-1} d \in G_{X}^{2 \ell}, \quad c \bar{c} \in G_{X}
$$

so $\bar{c}^{-1} \bar{V} \bar{c}=(c \bar{c})^{-1} d\left(d^{-1} c \bar{V} c^{-1} d\right) d^{-1}(c \bar{c}) \in G_{X}^{2 \ell}$, or equivalently,

(i) $\bar{V} \in\left(\bar{c} G_{X} \bar{c}^{-1}\right)^{2 \ell}=\left(G_{\operatorname{Ad}(\bar{c})(X)}\right)^{2 \ell}=\left(G_{\bar{X}}\right)^{2 \ell}$,

where $\bar{X}=-\operatorname{Ad}(\bar{c})(X)$. We also have

(ii) $\bar{c}^{-1}(\bar{c} c) \bar{c}=c \bar{c} \in G_{X}$, i.e., $\bar{c} c \in G_{\operatorname{Ad}(\bar{c})(X)}=G_{\bar{X}}$.

On the other hand,

(iii) $\bar{c}^{-1} \bar{d} \bar{c}=(c \bar{c})^{-1} \exp (-X / 2) \mathfrak{m}(V) d^{-1}(c \bar{c}) \in G_{X}$, i.e., $\bar{d} \in G_{\bar{X}}$. 
(iv) $V \in\left(G_{X}\right)^{2 \ell}$, so $\bar{c} V \bar{c}^{-1} \in\left(G_{\operatorname{Ad}(\bar{c}) X}\right)^{2 \ell}=\left(G_{\bar{X}}\right)^{2 \ell}$, and thus

$$
\bar{d}^{-1} \bar{c} V \bar{c}^{-1} \bar{d} \in\left(G_{\bar{X}}\right)^{2 \ell}
$$

To summarize, we have

$$
V, \bar{d}^{-1} \bar{c} V \bar{c}^{-1} \bar{d} \in\left(G_{X}\right)^{2 \ell}, \quad \bar{d}^{-1}, \bar{c} c \in G_{\bar{X}}
$$

We also have

$$
\begin{aligned}
\mathfrak{m}(\bar{V}) & =\bar{c} d \exp (-X / 2) \bar{c}^{-1} \bar{d}=\bar{c} \exp (-X / 2) d \bar{c}^{-1} \bar{d} \\
& =\exp (\bar{X} / 2) \bar{c} d \bar{c}^{-1} \bar{d} \\
\mathfrak{m}(V) & =\exp (X / 2) c \bar{d} c^{-1} d \\
& =c \bar{d} \cdot \bar{d}^{-1}(\bar{c} c)^{-1} \bar{c} \exp (X / 2) \bar{c}^{-1}(\bar{c} c) \cdot \bar{d} \cdot c^{-1} d ; \\
& =c \bar{d} \exp \left(\operatorname{Ad}\left(\bar{d}^{-1}\right) \circ \operatorname{Ad}\left((\bar{c} c)^{-1}\right)(-\bar{X} / 2) c^{-1} d\right. \\
& =c \bar{d} \exp (-\bar{X} / 2) c^{-1} d
\end{aligned}
$$

where we have used $\bar{d}^{-1},(\bar{c} c)^{-1} \in G_{\bar{X}}$ in the last equality. By (4.8)-(4.10),

$$
\tau(V, d, c, \bar{V}, \bar{d}, \bar{c}, X)=(\bar{V}, \bar{d}, \bar{c}, V, d, c, \bar{X}) \in Z_{\mathrm{YM}}^{\ell, 2}(G)
$$

This proves

$$
\tau\left(Z_{\mathrm{YM}}^{\ell, 2}(G)\right) \subset Z_{\mathrm{YM}}^{\ell, 2}(G)
$$

It remains to show that $\tau \circ \tau: Z_{\mathrm{YM}}^{\ell, i}(G) \rightarrow Z_{\mathrm{YM}}^{\ell, i}(G)$ is the identity map. We first consider the case $i=1$ : given $(V, c, \bar{V}, \bar{c}, X) \in Z_{\mathrm{YM}}^{\ell, 1}(G)$,

$$
\begin{aligned}
\tau \circ \tau(V, c, \bar{V}, \bar{c}, X) & =\tau(\bar{V}, \bar{c}, V, c,-\operatorname{Ad}(\bar{c})(X)=(V, c, \bar{V}, \bar{c}, \operatorname{Ad}(c \bar{c})(X)) \\
& =(V, c, \bar{V}, \bar{c}, X)
\end{aligned}
$$

where we have used $c \bar{c} \in G_{X}$. The case $i=2$ can be proved in the same way.

Thus we have

$$
\tau\left(Z_{\mathrm{YM}}^{\ell, i}(G)\right) \subset Z_{\mathrm{YM}}^{\ell, i}(G), \quad Z_{\mathrm{YM}}^{\ell, i}(G)=\tau \circ \tau\left(Z_{\mathrm{YM}}^{\ell, i}(G)\right) \subset \tau\left(Z_{\mathrm{YM}}^{\ell, i}(G)\right)
$$




\subsection{Representation varieties for nonorientable surfaces}

From the above discussion, we have

$$
\bigcup_{P \in \operatorname{Prin}_{G}\left(\Sigma_{i}^{\ell}\right)} \mathcal{N}(P) / \mathcal{G}(P) \cong Z_{\mathrm{YM}}^{\ell, i}(G)^{\tau} /(G \times G)^{\tau}
$$

for $i=1,2$, where $\tau: G \times G \rightarrow G \times G$ is given by $\left(g_{1}, g_{2}\right) \mapsto\left(g_{2}, g_{1}\right)$. We now relate the right hand side of (4.11) to representations of the super central extension $\Gamma_{\mathbb{R}}\left(\Sigma_{i}^{\ell}\right)$ of $\pi_{1}\left(\Sigma_{i}^{\ell}\right)$.

$\Gamma_{\mathbb{R}}\left(\Sigma_{1}^{\ell}\right)$ is generated by

$$
A_{1}, B_{1}, \ldots, A_{\ell}, B_{\ell}, C, J_{r}
$$

where $r \in \mathbb{R}$, with relations

(1) $J_{r_{1}} J_{r_{2}}=J_{r_{1}+r_{2}}$;

(2) $A_{i} J_{r} A_{i}^{-1}=B_{i} J_{r} B_{i}^{-1}=J_{r}, \quad i=1, \ldots, \ell, \quad r \in \mathbb{R}$;

(3) $C J_{r} C^{-1}=J_{-r}, \quad r \in \mathbb{R}$;

(4) $\prod_{i=1}^{\ell}\left[A_{i}, B_{i}\right]=J_{1} C^{2}$.

Let $\rho: \Gamma_{\mathbb{R}}\left(\Sigma_{1}^{\ell}\right) \rightarrow G$ be a group homomorphism. From relation (1) we must have $\rho\left(J_{r}\right)=\exp (r X)$ for some $X \in \mathfrak{g}$. From relation (2) we must have $\rho\left(A_{i}\right), \rho\left(B_{i}\right) \in G_{X}$. From relation (3) we have $\operatorname{Ad}(\rho(C))(X)=-X$. Combined with relation $(4), \operatorname{Hom}\left(\Gamma_{\mathbb{R}}\left(\Sigma_{1}^{\ell}\right), G\right)$ can be identified with

$$
\begin{aligned}
X_{\mathrm{YM}}^{\ell, 1}(G) & =\left\{(V, c, X) \in G^{2 \ell+1} \times \mathfrak{g} \mid V \in\left(G_{X}\right)^{2 \ell},\right. \\
\operatorname{Ad}(c)(X) & \left.=-X, \mathfrak{m}(V)=\exp (X) c^{2}\right\}
\end{aligned}
$$

There is a homeomorphism $X_{\mathrm{YM}}^{\ell, 1}(G) \stackrel{\cong}{\longrightarrow} Z_{\mathrm{YM}}^{\ell, 1}(G)^{\tau}$ given by

$$
(V, c, X) \longmapsto(V, c, V, c, 2 X), \quad V \in G^{2 \ell}, c \in G, X \in \mathfrak{g} .
$$

There is an inclusion $X_{\text {flat }}^{\ell, 1}(G) \hookrightarrow X_{\mathrm{YM}}^{\ell, 1}(G)$ given by $(V, c) \mapsto(V, c, 0)$.

$\Gamma_{\mathbb{R}}\left(\Sigma_{2}^{\ell}\right)$ is generated by

$$
A_{1}, B_{1}, \ldots, A_{\ell}, B_{\ell}, D, C, J_{r}
$$


where $r \in \mathbb{R}$, with relations

(1) $J_{r_{1}} J_{r_{2}}=J_{r_{1}+r_{2}}$;

(2) $A_{i} J_{r} A_{i}^{-1}=B_{i} J_{r} B_{i}^{-1}=D J_{r} D^{-1}=J_{r}, \quad i=1, \ldots, \ell, \quad r \in \mathbb{R}$;

(3) $C J_{r} C^{-1}=J_{-r}, \quad r \in \mathbb{R}$;

(4) $\prod_{i=1}^{\ell}\left[A_{i}, B_{i}\right]=J_{1} C D C^{-1} D$.

Let $\rho: \Gamma_{\mathbb{R}}\left(\Sigma_{2}^{\ell}\right) \rightarrow G$ be a group homomorphism. From relation (1) we must have $\rho\left(J_{r}\right)=\exp (r X)$ for some $X \in \mathfrak{g}$. From relation (2) we must have $\rho\left(A_{i}\right), \rho\left(B_{i}\right), \rho(D) \in G_{X}$. From relation (3) we have $\operatorname{Ad}(\rho(C))(X)=-X$. Combined with the relation $(4), \operatorname{Hom}\left(\Gamma_{\mathbb{R}}\left(\Sigma_{2}^{\ell}\right), G\right)$ can be identified with

$$
\begin{aligned}
X_{\mathrm{YM}}^{\ell, 2}(G) & =\left\{(V, d, c, X) \in G^{2 \ell+2} \times \mathfrak{g} \mid V \in\left(G_{X}\right)^{2 \ell}, d \in G_{X},\right. \\
\operatorname{Ad}(c)(X) & \left.=-X, \mathfrak{m}(V)=\exp (X) c d c^{-1} d\right\} .
\end{aligned}
$$

There is a homeomorphism $X_{\mathrm{YM}}^{\ell, 2}(G) \stackrel{\cong}{\longrightarrow} Z_{\mathrm{YM}}^{\ell, 2}(G)^{\tau}$ given by

$$
(V, d, c, X) \longmapsto(V, d, c, V, d, c, 2 X), \quad V \in G^{2 \ell}, d, c \in G, X \in \mathfrak{g} .
$$

There is an inclusion $X_{\text {flat }}^{\ell, 2}(G) \hookrightarrow X_{\mathrm{YM}}^{\ell, 2}(G)$ given by $(V, d, c) \mapsto(V, d, c, 0)$.

We obtain the following analog of Theorem 4.1 for nonorientable surfaces.

Theorem 4.6. There is a bijective correspondence between conjugacy classes of homomorphisms $\Gamma_{\mathbb{R}}(\Sigma) \rightarrow G$ and gauge equivalence classes of Yang-Mills $G$-connections over $\Sigma$. In other words, for $i=1,2$, we have

$$
\begin{gathered}
\bigcup_{P \in \operatorname{Prin}_{G}\left(\Sigma_{i}^{\ell}\right)} \mathcal{N}(P) / \mathcal{G}_{0}(P) \cong \operatorname{Hom}\left(\Gamma_{\mathbb{R}}\left(\Sigma_{i}^{\ell}\right), G\right) \cong X_{\mathrm{YM}}^{\ell, i}(G), \\
\bigcup_{P \in \operatorname{Prin}_{G}\left(\Sigma_{i}^{\ell}\right)} \mathcal{N}(P) / \mathcal{G}(P) \cong \operatorname{Hom}\left(\Gamma_{\mathbb{R}}\left(\Sigma_{i}^{\ell}\right), G\right) / G \cong X_{\mathrm{YM}}^{\ell, i}(G) / G,
\end{gathered}
$$

where $g \in G$ acts on $G^{2 \ell+1} \times \mathfrak{g}$ by

$$
g \cdot(V, c, X)=\left(g V g^{-1}, g c g^{-1}, \operatorname{Ad}(g)(X)\right),
$$

and on $G^{2 \ell+2} \times \mathfrak{g}$ by

$$
g \cdot(V, d, c, X)=\left(g V g^{-1}, g d g^{-1}, g c g^{-1}, \operatorname{Ad}(g)(X)\right)
$$




\subsection{Extended moduli spaces}

The representation variety $X_{\mathrm{YM}}^{\ell, 0}(G)$ is a subset of Lisa Jeffrey's extended moduli space [15]. In this subsection, we define extended moduli spaces for nonorientable surfaces.

Let $\Sigma_{0}^{\ell, r}$ be the compact, connected, orientable surface with $\ell$ handles and $r$ boundary components $S_{1}, \ldots, S_{r}$ with coordinates $\left(s_{1}, \ldots, s_{r}\right) \in \mathbb{R} / \mathbb{Z}$. Let $\Sigma_{1}^{\ell, r}$ be the connected sum of $\Sigma_{0}^{\ell}$ and $\mathbb{R P}^{2}$, and let $\Sigma_{2}^{\ell, r}$ be the connected sum of $\Sigma_{0}^{\ell}$ and a Klein bottle. The following discussion is a straightforward generalization of the case $i=0$ in [15].

Suppose that $r>0$ and $i=0,1,2$. Then any principal $G$-bundle $P$ over $\Sigma_{i}^{\ell, r}$ is topologically trivial. Let $\mathcal{A}(P)$ be the space of smooth connections on $P$. Then

$$
\mathcal{A}(P) \cong \Omega_{\Sigma_{i}^{\ell, r}}^{1}(\mathfrak{g})
$$

Define

$$
\begin{aligned}
\mathcal{A}_{G}^{\mathfrak{g}}\left(\Sigma_{i}^{\ell, r}\right)= & \left\{A \in \mathcal{A}(P)|F(A)=0, A|_{U_{j}}=X_{j} d s_{j}\right. \text { on some open } \\
& \text { neighborhood } \left.U_{j} \text { of } S_{j} \text { for some } X_{j} \in \mathfrak{g}, j=1, \ldots, r\right\}
\end{aligned}
$$

and define the compactly supported gauge group

$$
\begin{aligned}
\mathcal{G}^{c}\left(\Sigma_{i}^{\ell, r}\right)=\left\{s: \Sigma_{i}^{\ell, r} \rightarrow G \mid\right. & s(x)=e \text { for } x \in U, \text { where } U \text { is an open } \\
& \text { neighborhood of } \left.\partial \Sigma_{i}^{\ell, r}\right\} .
\end{aligned}
$$

We define a moduli space

$$
\mathcal{M}_{G}^{\mathfrak{g}}\left(\Sigma_{i}^{\ell, r}\right)=\mathcal{A}_{F}^{\mathfrak{g}}\left(\Sigma_{i}^{\ell, r}\right) / \mathcal{G}^{c}\left(\Sigma_{i}^{\ell, r}\right),
$$

and introduce representation varieties

$$
\begin{aligned}
\mathcal{N}_{G}^{\mathfrak{g}}\left(\Sigma_{0}^{\ell, r}\right)= & \left\{\left(V, k_{2}, \ldots, k_{r}, X_{1}, \ldots, X_{r} \in G^{2 \ell} \times G^{r-1} \times \mathfrak{g}^{r} \mid\right.\right. \\
& \left.\mathfrak{m}(V)=\exp \left(X_{1}\right) \exp \left(\operatorname{Ad}\left(k_{2}\right) X_{2}\right) \cdots \exp \left(\operatorname{Ad}\left(k_{r}\right) X_{r}\right)\right\}, \\
\mathcal{N}_{G}^{\mathfrak{g}}\left(\Sigma_{1}^{\ell, r}\right)= & \left\{\left(V, c, k_{2}, \ldots, k_{r}, X_{1}, \ldots, X_{r} \in G^{2 \ell} \times G^{r} \times \mathfrak{g}^{r} \mid\right.\right. \\
& \left.\mathfrak{m}(V)=\exp \left(X_{1}\right) \exp \left(\operatorname{Ad}\left(k_{2}\right) X_{2}\right) \cdots \exp \left(\operatorname{Ad}\left(k_{r}\right) X_{r}\right) c^{2}\right\}, \\
\mathcal{N}_{G}^{\mathfrak{g}}\left(\Sigma_{2}^{\ell, r}\right)= & \left\{\left(V, d, c, k_{2}, \ldots, k_{r}, X_{1}, \ldots, X_{r} \in G^{2 \ell} \times G^{r+1} \times \mathfrak{g}^{r} \mid\right.\right. \\
& \left.\left.\mathfrak{m}(V)=\exp \left(X_{1}\right) \exp \left(\operatorname{Ad}\left(k_{2}\right) X_{2}\right)\right) \cdots \exp \left(\operatorname{Ad}\left(k_{r}\right) X_{r}\right) c d c^{-1} d\right\} .
\end{aligned}
$$


where $\mathfrak{m}\left(a_{1}, b_{1}, \ldots, a_{\ell}, b_{\ell}\right)=\prod_{i=1}^{\ell}\left[a_{i}, b_{i}\right]$ as before. In particular,

$$
\begin{aligned}
& \mathcal{N}_{G}^{\mathfrak{g}}\left(\Sigma_{0}^{\ell, 1}\right)=\left\{(V, X) \in G^{2 \ell} \times \mathfrak{g} \mid \mathfrak{m}(V)=\exp (X)\right\}, \\
& \mathcal{N}_{G}^{\mathfrak{g}}\left(\Sigma_{1}^{\ell, 1}\right)=\left\{(V, c, X) \in G^{2 \ell+1} \times \mathfrak{g} \mid \mathfrak{m}(V)=\exp (X) c^{2}\right\}, \\
& \mathcal{N}_{G}^{\mathfrak{g}}\left(\Sigma_{2}^{\ell, 1}\right)=\left\{(V, d, c, X) \in G^{2 \ell+2} \times \mathfrak{g} \mid \mathfrak{m}(V)=\exp (X) c d c^{-1} d\right\},
\end{aligned}
$$

So

$$
X_{\mathrm{YM}}^{\ell, i}(G) \subset \mathcal{N}_{G}^{\mathfrak{g}}\left(\Sigma_{i}^{\ell, 1}\right)
$$

The following statement follows from the proof of [15, Proposition 5.3]:

Proposition 4.7. Let $\ell \geq 0, r>0$ be integers, and let $i=0,1,2$. Then there is a homeomorphism

$$
\mathcal{M}_{G}^{\mathfrak{g}}\left(\Sigma_{i}^{\ell, r}\right) \cong \mathcal{N}_{G}^{\mathfrak{g}}\left(\Sigma_{i}^{\ell, r}\right)
$$

\section{Equivariant Morse stratification of space of connections}

In this section, we discuss the $\mathcal{G}$-equivariant Morse stratification and reduction for general compact connected Lie groups. As a byproduct, we reproduce and extend the results on connected components of the moduli space of flat connections over closed nonorientable surfaces $[10,11]$.

\subsection{Morse stratification with involution}

Let $(M, g)$ be a Riemannian manifold. Let $f: M \rightarrow \mathbb{R}$ be a smooth function, and let $\phi_{t}$ be the gradient flow of $f$. Suppose that the gradient flow is defined for any time $t \in \mathbb{R}$ and the limits

$$
\lim _{t \rightarrow \infty} \phi_{t}(x), \quad \lim _{t \rightarrow-\infty} \phi_{t}(x)
$$

exist for any $x \in M$. Let $N$ be the set of critical points of $f$, and let

$$
N=\bigcup_{\mu \in \Lambda} N_{\mu}
$$

be the union of connected components. Suppose that each $N_{\mu}$ is a closed nondegenerate critical submanifold of $M$. Given a critical subset $N_{\mu}$, define 
its stable manifold $S_{\mu}$ and unstable manifold $U_{\mu}$ by

$$
S_{\mu}=\left\{x \in M \mid \lim _{t \rightarrow-\infty} \phi_{t}(x) \in N_{\mu}\right\}, \quad U_{\mu}=\left\{x \in M \mid \lim _{t \rightarrow+\infty} \phi_{t}(x) \in N_{\mu}\right\} .
$$

Then

$$
M=\bigcup_{\mu \in \Lambda} S_{\mu}
$$

is a disjoint union of Morse strata. We assume that each $S_{\mu}$ is a submanifold of $M$.

Suppose that $f$ is invariant under some isometric involution $\tau: M \rightarrow M$. By Lemma 3.2, $\tau$ induces an involution $\tau_{0}: \Lambda \rightarrow \Lambda$ such that

$$
\tau\left(N_{\mu}\right)=N_{\tau_{0}(\mu)}, \quad \tau\left(S_{\mu}\right)=S_{\tau_{0}(\mu)}, \quad \tau\left(U_{\mu}\right)=U_{\tau_{0}(\mu)}
$$

Proposition 5.1. Let $(M, \omega, J)$ be an almost Kähler manifold with an antisymplectic, anti-holomorphic involution $\tau: M \rightarrow M$. Suppose that $f: M \rightarrow$ $\mathbb{R}$ is a $\tau$-invariant smooth function. Suppose that $N_{\mu}$ is a closed subset of $M$ and a connected component of the set of critical points $N$ of $f$. Suppose that the set

$$
S_{\mu}=\left\{x \in M \mid \lim _{t \rightarrow-\infty} \phi_{t}(x) \in N_{\mu}\right\}
$$

is an almost complex submanifold of $M$. If

$$
S_{\mu}^{\tau}=\left\{x \in M^{\tau} \mid \lim _{t \rightarrow-\infty} \phi_{t}(x) \in N_{\mu}^{\tau}\right\}
$$

is nonempty, then $S_{\mu}^{\tau}$ is the stable manifold of $\mathcal{N}_{\mu}^{\tau}$ with respect to $f^{\tau}$, and the real codimension of $S_{\mu}^{\tau}$ in $M^{\tau}$ is equal to the complex codimension of $S_{\mu}$ in $M$.

Note that $M^{\tau}$ and $S_{\mu}^{\tau}$ are not necessarily connected.

\subsection{Morse stratification and Morse inequalities}

Let $\Sigma$ be a closed, compact, connected surface. Then $\Sigma$ is diffeomorphic to $\Sigma_{i}^{\ell}$ for some integer $\ell \geq 0$ and $i \in\{0,1,2\}$. Recall that $\chi\left(\Sigma_{i}^{\ell}\right)=2-2 \ell-i$. 
Let $G$ be a compact, connected Lie group. By Theorem 4.1 and Theorem 4.6, we know that

$$
\begin{gathered}
\bigcup_{P \in \operatorname{Prin}_{G}(\Sigma)} \mathcal{N}(P) / \mathcal{G}_{0}(P) \cong \operatorname{Hom}\left(\Gamma_{\mathbb{R}}(\Sigma), G\right), \\
\bigcup_{P \in \operatorname{Prin}_{G}(\Sigma)} \mathcal{N}(P) / \mathcal{G}(P) \cong \operatorname{Hom}\left(\Gamma_{\mathbb{R}}(\Sigma), G\right) / G .
\end{gathered}
$$

Let $\operatorname{Hom}\left(\Gamma_{\mathbb{R}}(\Sigma), G\right)_{P} \subset \operatorname{Hom}\left(\Gamma_{\mathbb{R}}(\Sigma), G\right)$ be the subset corresponding to $P \in$ $\operatorname{Prin}_{G}(\Sigma)$, so that

$$
\begin{gathered}
\operatorname{Hom}\left(\Gamma_{\mathbb{R}}(\Sigma), G\right)=\bigcup_{P \in \operatorname{Prin}_{G}(\Sigma)} \operatorname{Hom}\left(\Gamma_{\mathbb{R}}(\Sigma), G\right)_{P}, \\
\mathcal{N}(P) / \mathcal{G}_{0}(P) \cong \operatorname{Hom}\left(\Gamma_{\mathbb{R}}(\Sigma), G\right)_{P}, \quad \mathcal{N}(P) / \mathcal{G}(P) \cong \operatorname{Hom}\left(\Gamma_{\mathbb{R}}(\Sigma), G\right)_{P} / G .
\end{gathered}
$$

For a fixed topological principal $G$-bundle $P$ over $\Sigma$, let

$$
\left\{\mathcal{N}_{\tilde{\mu}}(P) \mid \tilde{\mu} \in \pi_{0}(\mathcal{N}(P))\right\}
$$

be the set of connected components of $\mathcal{N}(P)$. Let $\mathcal{G}(P)^{\prime}$ be the connected component of the identity of $\mathcal{G}(P)$. Then $\mathcal{G}(P)^{\prime}$ is a normal subgroup of $\mathcal{G}(P)$, and the discrete set $\pi_{0}(\mathcal{G}(P))$ can be identified with the group $\mathcal{G}(P) / \mathcal{G}(P)^{\prime}$. The action of $\mathcal{G}(P)$ on $\mathcal{N}(P)$ is continuous, and induces an action of $\pi_{0}(\mathcal{G}(P)) \cong \mathcal{G}(P) / \mathcal{G}(P)^{\prime}$ on $\pi_{0}(\mathcal{N}(P))$. Define

$$
\Lambda=\pi_{0}(\mathcal{N}(P)) / \pi_{0}(\mathcal{G}(P)) .
$$

Remark 5.2. When $\Sigma$ is orientable, the action of $\pi_{0}(\mathcal{G}(P))$ on $\pi_{0}(\mathcal{N}(P))$ is trivial by the results in [1], so $\Lambda=\pi_{0}(\mathcal{N}(P))$.

Let $\pi: \pi_{0}(\mathcal{N}(P)) \rightarrow \Lambda=\pi_{0}(\mathcal{N}(P)) / \pi_{0}(\mathcal{G}(P))$ be the projection. Given $\mu \in \Lambda$, define

$$
\mathcal{N}_{\mu}(P)=\bigcup_{\tilde{\mu} \in \pi^{-1}(\mu)} \mathcal{N}_{\tilde{\mu}}(P) .
$$

Then $\mathcal{G}(P)$ acts on $\mathcal{N}_{\mu}(P)$, and $\mathcal{N}_{\mu}(P) / \mathcal{G}(P)$ is connected. Note that the quotient $\mathcal{G}(P) / \mathcal{G}_{0}(P)=G$ is connected, so

$$
V_{\mu}(P) \stackrel{\text { def }}{=} \mathcal{N}_{\mu}(P) / \mathcal{G}_{0}(P)
$$

is connected.

$$
\operatorname{Hom}\left(\Gamma_{\mathbb{R}}(\Sigma), G\right)_{P}=\bigcup_{\mu \in \Lambda} V_{\mu}(P)
$$


is a disjoint union of connected components. Each $\mathcal{N}_{\mu}(P)$ is a closed subset of $\mathcal{N}(P)$, thus of $\mathcal{A}(P)$. Define

$$
\mathcal{A}_{\mu}(P)=\left\{A \in \mathcal{A}(P) \mid \lim _{t \rightarrow-\infty} \phi_{t}(A) \in \mathcal{N}_{\mu}(P)\right\},
$$

where $\phi_{t}$ is the gradient flow of $L_{P}$. The limit exists by results in [6] and [24]. Notice that $L_{P}$ is constant on each $\mathcal{N}_{\mu}(P)$, and $L_{P}$ achieves its minimum on $\mathcal{N}_{\mu}(P)$ within $\mathcal{A}_{\mu}(P)$. Each $\mathcal{A}_{\mu}(P)$ is a submanifold of $\mathcal{A}(P)$, and the map $\mathcal{A}_{\mu}(P) \rightarrow \mathcal{N}_{\mu}(P)$ given by $A \mapsto \lim _{t \rightarrow-\infty} \phi_{t}(A)$ is a $\mathcal{G}(P)$-equivariant deformation retraction. We have

$$
\mathcal{A}(P)=\bigcup_{\mu \in \Lambda} \mathcal{A}_{\mu}(P)
$$

is a smooth stratification. This stratification is $\mathcal{G}(P)$-equivariant in the sense that $\mathcal{G}(P)$ acts on each stratum. We call $(5.1)$ the $\mathcal{G}(P)$-equivariant Morse stratification of $\mathcal{A}(P)$ with respect to $L_{P}$.

Remark 5.3. Given $\tilde{\mu} \in \pi_{0}(\mathcal{N}(P))$, define

$$
\mathcal{A}_{\tilde{\mu}}(P)=\left\{A \in \mathcal{A}(P) \mid \lim _{t \rightarrow-\infty} \phi_{t}(A) \in \mathcal{N}_{\tilde{\mu}}(P)\right\} .
$$

Then $\mathcal{A}_{\tilde{\mu}}$ is a connected submanifold of $\mathcal{A}(P)$, and is a Morse stratum of $L_{P}$. When $\Sigma$ is orientable, the Morse stratification coincides with the $\mathcal{G}(P)$ equivariant Morse stratification by Remark 5.2; when $\Sigma$ is nonorientable, a priori the Morse stratification can be finer than the $\mathcal{G}(P)$-equivariant Morse stratification.

We now assume that $\chi(\Sigma)<0$. Let $\mathcal{N}_{s s}$ be the set where the Yang-Mills functional $L_{P}$ achieves absolute minimum. Then $\mathcal{N}_{s s}$ is connected (by results in [1] when $\Sigma$ is orientable, and by Theorem 5.6 when $\Sigma$ is nonorientable). $\mathcal{N}_{s s}$ is the unique connected component of the critical set $\mathcal{N}(P)$ with zero Morse index, and its stable manifold $\mathcal{A}_{s s}$ is the unique codimension zero Morse stratum (which is also a $\mathcal{G}(P)$-equivariant Morse stratum). Define

$$
V_{s s}(P)=\mathcal{N}_{s s} / \mathcal{G}_{0}(P) .
$$

Then $V_{s s}(P)$ is connected.

When the obstruction class $o(P) \in H^{2}\left(\Sigma, \pi_{1}(G)\right)$ is a torsion element (which is always true when $\Sigma$ is nonorientable), $\mathcal{N}_{s s}(P)=\mathcal{N}_{0}(P)$ is the 
space of flat connections on $P$, and

$$
V_{s s}(P) \cong \operatorname{Hom}\left(\pi_{1}(\Sigma), G\right)_{P},
$$

where $\operatorname{Hom}\left(\pi_{1}(\Sigma), G\right)_{P}$ is the connected component of $\operatorname{Hom}\left(\pi_{1}(\Sigma), G\right)$ associated to the topological $G$-bundle $P$ (see $[1,11])$.

In general, we are interested in the cohomology of the moduli space $\mathcal{M}(P)$ of gauge equivalence classes of minimal Yang-Mills connections on $P$. More explicitly,

$$
\mathcal{M}(P) \stackrel{\text { def }}{=} \mathcal{N}_{s s}(P) / \mathcal{G}(P) \cong V_{s s}(P) / G
$$

When $\mathcal{M}(P)$ is smooth and $\mathcal{G}(P)$ acts on $\mathcal{N}_{s s}(P)$ freely, we have

$$
H^{*}(\mathcal{M}(P) ; \mathbb{Q}) \cong H_{\mathcal{G}(P)}^{*}\left(\mathcal{N}_{s s}(P) ; \mathbb{Q}\right) \cong H_{G}^{*}\left(V_{s s}(P) ; \mathbb{Q}\right)
$$

The deformation retraction $r: \mathcal{A}_{\mu}(P) \rightarrow \mathcal{N}_{\mu}(P)$ given by the gradient flow of $L_{P}$ is $\mathcal{G}(P)$-equivariant, thus the following equivariant pairs are equivalent for the purpose of (singular) equivariant cohomology:

$$
\begin{aligned}
\left(\mathcal{A}_{\mu}(P), \mathcal{G}(P)\right) \sim & \left(\mathcal{N}_{\mu}(P), \mathcal{G}(P)\right) \sim\left(\mathcal{N}_{\mu}(P) / \mathcal{G}_{0}(P), \mathcal{G}(P) / \mathcal{G}_{0}(P)\right) \\
& \sim\left(V_{\mu}(P), G\right) .
\end{aligned}
$$

In other words, we have the following homotopy equivalences of homotopic orbit spaces:

$$
\mathcal{A}_{\mu}(P)^{h \mathcal{G}(P)} \sim \mathcal{N}_{\mu}(P)^{h \mathcal{G}(P)} \sim V_{\mu}(P)^{h G} .
$$

As a consequence, we have the following isomorphisms of (singular) equivariant cohomology:

$$
H_{\mathcal{G}(P)}^{*}\left(\mathcal{A}_{\mu}(P) ; \mathbb{Q}\right) \cong H_{\mathcal{G}(P)}^{*}\left(\mathcal{N}_{\mu}(P) ; \mathbb{Q}\right) \cong H_{G}^{*}\left(V_{\mu}(P) ; \mathbb{Q}\right) .
$$

Let $K$ be a field and let

$$
P_{t}^{\mathcal{G}(P)}\left(\mathcal{A}_{\mu}(P) ; K\right)=\sum_{i \geq 0} t^{i} \operatorname{dim} H_{\mathcal{G}(P)}^{i}\left(\mathcal{A}_{\mu}(P) ; K\right)
$$

be the equivariant Poincaré series. Let

$$
\begin{aligned}
M_{t}^{\mathcal{G}(P)}\left(L_{P} ; K\right) & =\sum_{\mu \in \Lambda} t^{\lambda_{\mu}} P_{t}^{\mathcal{G}(P)}\left(\mathcal{A}_{\mu}(P) ; K\right) \\
& =P_{t}^{\mathcal{G}(P)}\left(\mathcal{A}_{s s}(P) ; K\right)+\sum_{\mu \in \Lambda^{\prime}} t^{\lambda_{\mu}} P_{t}^{\mathcal{G}(P)}\left(\mathcal{A}_{\mu}(P) ; K\right)
\end{aligned}
$$


be the $\mathcal{G}(P)$-equivariant Morse series of $L_{P}: \mathcal{A}(P) \rightarrow \mathbb{R}$, where $\lambda_{\mu}$ is the real codimension of the stratum $\mathcal{A}_{\mu}(P)$ in $\mathcal{A}(P)$ and $\Lambda^{\prime}=\left\{\mu \in \Lambda \mid \lambda_{\mu}>0\right\}$.

The Morse stratification is smooth and $\mathcal{G}(P)$-equivariant, so we have equivariant Morse inequalities [6,17]: there exists a power series $R_{K}(t)$ with nonnegative coefficients such that

$$
M_{t}^{\mathcal{G}(P)}\left(L_{P} ; K\right)-P_{t}^{\mathcal{G}(P)}(\mathcal{A}(P) ; K)=(1+t) R_{K}(t)
$$

A priori (5.4) holds for $K=\mathbb{Z} / 2 \mathbb{Z}$. If the normal bundle $\nu\left(\mathcal{A}_{\mu}\right)$ of $\mathcal{A}_{\mu}(P)$ in $\mathcal{A}(P)$ is orientable, then (5.4) holds for any $K$. When $\Sigma$ is orientable, $\nu\left(\mathcal{A}_{\mu}\right)$ is a complex vector bundle and has a canonical orientation.

Equation (5.2) and (5.3) imply

$$
\begin{aligned}
M_{t}^{\mathcal{G}(P)}\left(L_{P} ; K\right) & =\sum_{\mu \in \Lambda} t^{\lambda_{\mu}} P_{t}^{G}\left(V_{\mu}(P) ; K\right) \\
& =P_{t}^{G}\left(V_{s s}(P) ; K\right)+\sum_{\mu \in \Lambda^{\prime}} t^{\lambda_{\mu}} P_{t}^{G}\left(V_{\mu}(P) ; K\right)
\end{aligned}
$$

where now we consider the equivariant cohomology of $V_{\mu}(P)$, the representation variety, which is finite dimensional but singular, as opposed to $\mathcal{A}_{\mu}(P)$, which is smooth but infinite dimensional.

\subsection{Equivariant Poincaré series}

When $\Sigma$ is orientable, Atiyah and Bott [1] provide an algorithm to compute the equivariant Poincaré series

$$
P_{t}^{\mathcal{G}(P)}\left(\mathcal{A}_{s s}(P) ; \mathbb{Q}\right)=P_{t}^{\mathcal{G}(P)}\left(\mathcal{N}_{s s}(P) ; \mathbb{Q}\right)
$$

We now outline this algorithm.

Let $G^{\mathbb{C}}$ be the complexification of $G$. Then $G^{\mathbb{C}}$ is a connected reductive algebraic group over $\mathbb{C}$. (For example, if $G=U(n)$ then $G^{\mathbb{C}}=G L(n, \mathbb{C})$.) There is a bijection

$$
\operatorname{Prin}_{G}(\Sigma) \longrightarrow \operatorname{Prin}_{G^{\mathbb{C}}}(\Sigma)
$$

given by $P \mapsto P \times_{G} G^{\mathbb{C}}$.

We can consider $\mathcal{C}(\xi)$, the space of $(0,1)$-connections on $\xi=P \times_{G} G^{\mathbb{C}}$. Using Harder-Narasimhan filtration, Atiyah-Bott gave a stratification for 
$\mathcal{C}(\xi)$ by

$$
\mathcal{C}(\xi)=\bigcup_{\mu \in \Xi} \mathcal{C}_{\mu}(\xi)
$$

where $\mu$ denotes Harder-Narasimhan type $\mu$ and each $\mathcal{C}_{\mu}$ is connected [1, Chapter 7, 10].

Atiyah-Bott proved that the Harder-Narasimhan stratification is $\mathcal{G}^{\mathbb{C}}$. equivariantly perfect over $\mathbb{Q}$, i.e.,

$$
P_{t}^{\mathcal{G}^{\mathbb{C}}}(\mathcal{C}(\xi) ; \mathbb{Q})=P_{t}^{\mathcal{G}^{\mathbb{C}}}\left(\mathcal{C}_{s s}(\xi) ; \mathbb{Q}\right)+\sum_{\mu \in \Xi^{\prime}} t^{\lambda_{\mu}} P_{t}^{\mathcal{G}^{\mathbb{C}}}\left(\mathcal{C}_{\mu}(\xi) ; \mathbb{Q}\right)
$$

where $\mathcal{C}_{s s}$ is the semi-stable stratum, $\Xi^{\prime}=\left\{\mu \in \Xi \mid \mathcal{C}_{\mu}(\xi) \neq \mathcal{C}_{s s}(\xi)\right\}$. Now $\mathcal{C}(\xi)$ is contractible, so

$$
H_{\mathcal{G}^{\mathbb{C}}}^{*}(\mathcal{C}(\xi) ; \mathbb{Z})=H_{\mathcal{G}^{\mathbb{C}}}^{*}(\mathrm{pt} ; \mathbb{Z})=H^{*}\left(B \mathcal{G}^{\mathbb{C}} ; \mathbb{Z}\right)=H^{*}(B \mathcal{G}(P) ; \mathbb{Z})
$$

Thus

$$
P_{t}(B \mathcal{G}(P) ; \mathbb{Q})=P_{t}^{\mathcal{G}^{\mathbb{C}}}\left(\mathcal{C}_{s s}(\xi) ; \mathbb{Q}\right)+\sum_{\mu \in \Xi^{\prime}} t^{\lambda_{\mu}} P_{t}^{\mathcal{G}^{\mathbb{C}}}\left(\mathcal{C}_{\mu}(\xi) ; \mathbb{Q}\right)
$$

On the other hand, there is a natural isomorphism $i: \mathcal{C}(\xi) \rightarrow \mathcal{A}(P)$, and it was proven in [6] (conjectured by [1]) that the Harder-Narasimhan stratification coincides with the Morse stratification defined by $L_{P}$ as in Section 5.2, i.e. $\Xi \cong \pi_{0}(\mathcal{N}(P)) \cong \Lambda$. So the codimension of $\mathcal{C}_{\mu}$ in $\mathcal{C}$ equals to the codimension of $\mathcal{A}_{\mu}=i\left(\mathcal{C}_{\mu}\right)$ in $\mathcal{A}$. In particular, $\mathcal{A}_{s s}=i\left(\mathcal{C}_{s s}\right)$. The moduli space $\mathcal{M}(P)$ of minimal (central) Yang-Mills connections on $P$ can be identified with the moduli space of $S$-equivalence classes of semi-stable holomorphic structures on $\xi[1,25]$.

The equivariant perfectness of Harder-Narasimhan stratification $\cup \mathcal{C}_{\mu}$ now implies that

$$
\begin{aligned}
P_{t}^{\mathcal{G}}(\mathcal{A}(P) ; \mathbb{Q}) & =P_{t}^{\mathcal{G}^{\mathbb{C}}}(\mathcal{C}(\xi) ; \mathbb{Q})=\sum_{\mu \in \Xi} t^{\lambda_{\mu}} P_{t}^{\mathcal{G}^{\mathbb{C}}}\left(\mathcal{C}_{\mu}(\xi) ; \mathbb{Q}\right) \\
& =\sum_{\mu \in \Lambda} t^{\lambda_{\mu}} P_{t}^{\mathcal{G}}\left(\mathcal{A}_{\mu}(P) ; \mathbb{Q}\right)=M_{t}^{\mathcal{G}(P)}\left(L_{P} ; \mathbb{Q}\right)
\end{aligned}
$$

i.e., $R_{\mathbb{Q}}(t)=0$ and Morse stratification is $\mathcal{G}(P)$-equivariantly perfect:

$$
P_{t}(B \mathcal{G}(P) ; \mathbb{Q})=P_{t}^{G}\left(V_{s s}(P) ; \mathbb{Q}\right)+\sum_{\mu \in \Lambda^{\prime}} t^{\lambda_{\mu}} P_{t}^{G}\left(V_{\mu}(P) ; \mathbb{Q}\right)
$$


The equivariant pair $\left(V_{\mu}(P), G\right)$ can be reduced further (cf: [1, Section 10]):

$$
\left(V_{\mu}(P), G\right) \sim\left(V_{s s}\left(P_{\mu}\right), G_{\mu}\right)
$$

where $G_{\mu}$ is a compact Lie subgroup of $G$ with $\operatorname{dim}_{\mathbb{R}} G_{\mu}<\operatorname{dim}_{\mathbb{R}} G$, and $P_{\mu}$ is a principal $G_{\mu}$-bundle. So we have $P_{t}^{G}\left(V_{\mu}(P) ; \mathbb{Q}\right)=P_{t}^{G_{\mu}}\left(V_{s s}\left(P_{\mu}\right) ; \mathbb{Q}\right)$ and

$$
P_{t}(B \mathcal{G}(P) ; \mathbb{Q})=P_{t}^{G}\left(V_{s s}(P) ; \mathbb{Q}\right)+\sum_{\mu \in \Lambda^{\prime}} t^{\lambda_{\mu}} P_{t}^{G_{\mu}}\left(V_{s s}\left(P_{\mu}\right) ; \mathbb{Q}\right) .
$$

The left hand side of (5.10) has been computed (see [1, Theorem 2.15] and $\left[22\right.$, Theorem 3.3]). So $P_{t}^{G}\left(V_{s s}(P) ; \mathbb{Q}\right)$ can be computed recursively. The case $G=U(n)$ is particularly nice because

U1. $G_{\mu}$ is of the form $U\left(n_{1}\right) \times \cdots \times U\left(n_{r}\right)$, so the inductive reduction only involves type A classical groups.

U2. $H^{*}(B \mathcal{G}(P) ; \mathbb{Z})$ is torsion free, so $\operatorname{rank} H^{i}(B \mathcal{G}(P) ; \mathbb{Z})=\operatorname{dim}_{\mathbb{Q}} H^{i}(B \mathcal{G}(P)$; Q).

Neither U1 nor U2 is true for a general compact connected Lie group $G$.

Finally, we point out difficulties (N1-N4 below) in generalizing the above approach to nonorientable surfaces, and then end this subsection by a summary of our progress in resolving them.

N1. It is not clear if $\nu\left(\mathcal{A}_{\mu}\right)$ is orientable in general, so a priori Morse inequalities hold only for $\mathbb{Z} / 2 \mathbb{Z}$ :

$$
\begin{aligned}
P_{t}(B \mathcal{G}(P) ; \mathbb{Z} / 2 \mathbb{Z})= & P_{t}^{G}\left(V_{s s}(P) ; \mathbb{Z} / 2 \mathbb{Z}\right)+\sum_{\mu \in \Lambda^{\prime}} t^{\lambda_{\mu}} P_{t}^{G}\left(V_{\mu}(P) ; \mathbb{Z} / 2 \mathbb{Z}\right) \\
& -(1+t) R_{\mathbb{Z} / 2 \mathbb{Z}}(t) .
\end{aligned}
$$

N2. The left hand side of $(5.11)$ is difficult to compute when $H^{*}(B \mathcal{G}(P) ; \mathbb{Z})$ has 2-torsion elements. When $\Sigma$ is nonorientable, $H^{*}(B \mathcal{G}(P) ; \mathbb{Z})$ has 2-torsion elements even when $G=U(n)$. (We thank Paul Selick for pointing this out to us.)

N3. Suppose that for a particular $P$ we can prove that $\nu\left(\mathcal{A}_{\mu}\right)$ is orientable for all $\mu \in \Lambda$. We can consider rational cohomology

$$
P_{t}(B \mathcal{G}(P) ; \mathbb{Q})=P_{t}^{G}\left(V_{s s}(P) ; \mathbb{Q}\right)+\sum_{\mu \in \Lambda^{\prime}} t^{\lambda_{\mu}} P_{t}^{G}\left(V_{\mu}(P) ; \mathbb{Q}\right)-(1+t) R_{\mathbb{Q}}(t)
$$


Although $P_{t}(B \mathcal{G}(P) ; \mathbb{Q})$ is easier to handle than $P_{t}(B \mathcal{G}(P) ; \mathbb{Z} / 2 \mathbb{Z})$, it is tricky to compute $R_{\mathbb{Q}}(t)$, which is not necessarily zero.

N4. When the base $\Sigma$ of the principal $G$-bundle $P$ is nonorientable, we still have reduction $\left(V_{\mu}(P), G\right) \sim\left(V_{\mu}^{\prime}\left(P^{\prime}\right), G_{\mu}\right)$, where $G_{\mu}$ is a compact Lie subgroup of $G$ with $\operatorname{dim}_{\mathbb{R}} G_{\mu}<\operatorname{dim}_{\mathbb{R}} G$, but $V_{\mu}^{\prime}\left(P^{\prime}\right)$ is not of the form $V_{s s}\left(P_{\mu}\right)$, where $P_{\mu}$ a principal $G_{\mu}$-bundle over $\Sigma$.

We will describe equivariant Morse stratification and the reduction N4 for $G=U(n)$ in Section 7 of this paper, and for other classical groups in [12]. In a joint work with Ramras, we will prove that $\nu\left(\mathcal{A}_{\mu}\right)$ in N1 is orientable when $G=U(n)$, so we may consider $\mathbb{Q}$ coefficient as in N3.

\subsection{Connected components of moduli spaces of flat connections}

Let $G$ be any compact connected Lie group. Let $\Sigma$ be a closed, compact, connected, nonorientable surface, and let $\pi: \tilde{\Sigma} \rightarrow \Sigma$ be its orientable double cover. Let $P \rightarrow \Sigma$ be a principal $G$-bundle. By Proposition 2.8, the pull back principal $G$-bundle $\tilde{P}=\pi^{*} P \rightarrow \tilde{\Sigma}$ is topologically trivial, and there is an involution $\tilde{\tau}: \tilde{P} \rightarrow \tilde{P}$ which covers the deck transformation $\tau: \tilde{\Sigma} \rightarrow \tilde{\Sigma}$ such that $\tilde{P} / \tilde{\tau}=P$.

The involution $\tilde{\tau}$ induces an involution $\tilde{\tau}^{*}$ on the space $\mathcal{A}(\tilde{P})$ of connections on $\tilde{P}$. The space $\mathcal{A}(P)$ of connections on $P$ can be identified with the fixed locus $\mathcal{A}(\tilde{P})^{\tilde{\tau}^{*}}$. Each $\mathcal{G}(P)$-equivariant Morse stratum $\mathcal{A}_{\mu}(P)$ of $L_{P}$ is a union of connected components of $\mathcal{A}_{\mu}(\tilde{P})^{\tilde{\tau}^{*}}=\mathcal{A}_{\mu}(\tilde{P}) \cap \mathcal{A}(\tilde{P})^{\tilde{\tau}^{*}}$, where $\mathcal{A}_{\mu}(\tilde{P})$ is a Morse stratum in $\mathcal{A}(\tilde{P})$. The real codimension of $\mathcal{A}_{\mu}(P)$ in $\mathcal{A}(P)$ is equal to the complex codimension $d_{\mu}$ of $\mathcal{A}_{\mu}(\tilde{P})$ in $\mathcal{A}(\tilde{P})$ (see Proposition 5.1).

Let $\mathcal{N}_{0}(\tilde{P})$ and $\mathcal{N}_{0}(P)$ be the space of flat connections on $\tilde{P}$ and on $P$, respectively. Then $\mathcal{N}_{0}(P)=\mathcal{N}_{0}(\tilde{P})^{\tilde{\tau}^{*}}$. By discussion in Section 3.2, $\mathcal{N}_{0}(P)$ is nonempty. Let $\mathcal{A}_{s s}(P)=\mathcal{A}_{s s}(\tilde{P}) \cap \mathcal{A}(\tilde{P})^{\tilde{\tau}^{*}}$ be the stable manifold of $\mathcal{N}_{0}(P)=\mathcal{N}_{s s}(P)$, so that it is the union of all codimension zero Morse strata. By results in $[1], \mathcal{A}_{s s}(\tilde{P})$ is connected when $\chi(\tilde{\Sigma})<0$. We will show that $\mathcal{A}_{s s}(P)$ is connected when $\chi(\Sigma)<0$.

Proposition 5.4. Given two points $A_{0}, A_{1} \in \mathcal{A}_{s s}(P)$, there exists a smooth map $\gamma:[0,1] \rightarrow \mathcal{A}(P)$ such that $\gamma(0)=A_{0}, \gamma(1)=A_{1}$, and $\gamma$ is transversal to $\mathcal{A}_{\mu}(P)$ if $d_{\mu}>0$. In particular, $\gamma^{-1}\left(\mathcal{A}_{\mu}\right)$ is empty if $d_{\mu}>1$.

Proof. Let $\Omega=\Omega^{1}(\Sigma$, ad $P)$ be the vector space associated to the affine space $\mathcal{A}(P)$. Given $A_{0}, A_{1} \in \mathcal{A}_{s s}(P)$, define

$$
\Phi:[0,1] \times \Omega \rightarrow \mathcal{A}(P), \quad \Phi(t, a)=(1-t) A_{0}+t A_{1}+\sin (\pi t) a .
$$


Note that

$$
\Phi\left(\frac{1}{2}, a\right)=\frac{1}{2}\left(A_{0}+A_{1}\right)+a,
$$

so $\Phi$ is surjective. $d \Phi_{(t, a)}: \mathbb{R} \times \Omega \rightarrow \Omega$ is given by

$$
(u, b) \longmapsto\left(\left(A_{1}-A_{0}\right)+\pi \cos (\pi t) a\right) u+\sin (\pi t) b,
$$

where $u \in \mathbb{R}, b \in \Omega$. Given $a \in \Omega$ let $\gamma_{a}(t)=\Phi(t, a)$. Then

$$
d \Phi_{(t, a)}(u, b)=d\left(\gamma_{a}\right)_{t}(u)+\sin (\pi t) b
$$

We claim that $\Phi$ is transversal to $\mathcal{A}_{\mu}(P)$ for any $\mu \in \Lambda^{\prime}$. Fix $\lambda \in \Lambda^{\prime}$, we need to show that

$$
\operatorname{Im}\left(d \Phi_{(t, a)}\right)+T_{\Phi(t, a)} \mathcal{A}_{\mu}(P)=T_{\Phi(t, a)} \mathcal{A}(P)=\Omega
$$

for any $(t, a) \in \Phi^{-1}\left(\mathcal{A}_{\mu}(P)\right)$. Note that $\Phi(0, a)=A_{0} \in \mathcal{A}_{s s}$ and $\Phi(1, a)=$ $A_{1} \in \mathcal{A}_{s s}(P)$ for any $a \in \Omega$, so if $(t, a) \in \Phi^{-1}\left(\mathcal{A}_{\mu}(P)\right)$, we must have $0<t<$ 1. By (5.13), $\operatorname{Im}\left(d \Phi_{(t, a)}\right)=\Omega$ if $0<t<1$. So $\Phi$ is transversal to $\mathcal{A}_{\mu}(P)$ for any $\mu \in \Lambda^{\prime}$. We conclude that $\Phi^{-1}\left(\mathcal{A}_{\mu}(P)\right)$ is a submanifold of $[0,1] \times \Omega$ of codimension $d_{\mu}$; it is nonempty because $\Phi$ is surjective.

For any $\mu \in \Lambda^{\prime}$, we define $\pi_{\mu}: \Phi^{-1}\left(\mathcal{A}_{\mu}\right) \rightarrow \Omega$ by $(t, a) \mapsto a$. By Lemma 5.5, $a$ is a regular value of $\pi_{\mu}$ if and only of $\gamma_{a}:[0,1] \rightarrow \mathcal{A}(P)$ is transversal to $\mathcal{A}_{\mu}(P)$. Let $\Omega_{\mu}$ be the set of regular values of $\pi_{\mu}$. By the Sard-Smale theorem, $\Omega_{\mu}$ is residual in $\Omega$. So

$$
\Omega^{\prime}=\bigcap_{\mu \in \Lambda^{\prime}} \Omega_{\mu}
$$

is residual in $\Omega$. By the Baire category theorem, $\Omega^{\prime}$ is nonempty. For any $a \in \Omega^{\prime}, \gamma_{a}:[0,1] \rightarrow \mathcal{A}(P)$ has the desired properties.

Lemma 5.5. Let $X, Y, Z$ be linear spaces, and let $W$ be a linear subspace of $Z$. Let $L: X \times Y \rightarrow Z$ be a linear map such that $\operatorname{Im}(L)+W=Z$. Let $\pi: L^{-1}(W) \rightarrow Y$ be defined by $(x, y) \mapsto y$. Then $\pi$ is surjective if and only if $L(X \times\{0\})+W=Z$.

Proof. $\quad$ 1. Claim: $\pi$ is surjective $\Rightarrow L(X \times\{0\})+W=Z$.

Given any $z \in Z$, we have $z=L(x, y)+w$ for some $(x, y) \in X \times Y$ and $w \in W$. Since $\pi$ is surjective, there is $\left(x^{\prime}, y^{\prime}\right) \in X \times Y$ such that 
$L\left(x^{\prime}, y^{\prime}\right)=w^{\prime} \in W$ and $\pi\left(x^{\prime}, y^{\prime}\right)=y$. We have

$$
L(x, y)+w=z, \quad L\left(x^{\prime}, y^{\prime}\right)-w^{\prime}=0, \quad y=y^{\prime}
$$

SO

$$
z=L\left(x-x^{\prime}, 0\right)+\left(w+w^{\prime}\right) \in \operatorname{Im}(X \times\{0\})+W .
$$

2. Claim: $L(X \times\{0\})+W=Z \Rightarrow \pi$ is surjective.

Given any $y \in Y$, we have $L(0, y) \in Z$, so $L(0, y)=L(x, 0)+w$ for some $x \in X$ and $w \in W$. We have $L(-x, y)=w$, so $(-x, y) \in L^{-1}(W)$ and $\pi(-x, y)=y$.

We now assume that $\chi(\Sigma)<0$. The formula of $d_{\mu}$ is given by $[1,(10.7)]$ :

$$
d_{\mu}=\sum_{\alpha(\mu)>0}(\alpha(\mu)+\tilde{g}-1) \geq 0
$$

where $\tilde{g} \geq 2$ is the genus of $\tilde{\Sigma}$. Note that $d_{\mu} \geq 2$ if $d_{\mu} \neq 0$, so the real codimension of any lower stratum in $\mathcal{A}(P)$ is at least two. Since $\mathcal{N}_{0}(P)$ is a deformation retraction of $\mathcal{A}_{s s}(P)$, Proposition 5.4 implies the following.

Theorem 5.6. Let $\Sigma$ be a closed, compact, connected, nonorientable surface with negative Euler characteristic, or equivalently, $\Sigma=\Sigma_{i}^{\ell}$ where $i=1,2$ and $\ell \geq 1$. Let $G$ be a compact, connected Lie group, and let $P$ be a principal $G$-bundle over $\Sigma$. Then the space $\mathcal{N}_{0}(P)$ of flat connections on $P$ is nonempty and connected.

Corollary 5.7. Let $P$ be as in Theorem 5.6. Then the moduli space $\mathcal{M}(P)$ of gauge equivalence classes of flat connections on $P$ is nonempty and connected.

Note that the connectedness of $\mathcal{N}_{0}(P)$ implies the connectedness of $\mathcal{M}(P)=\mathcal{N}_{0}(P) / \mathcal{G}(P)$, but not vice versa, so in general Theorem 5.6 is stronger than Corollary 5.7.

Corollary 5.7 extends $[11$, Theorem 5.2$]$ to the case $\Sigma=4 \mathbb{R P}^{2}$. We thank the referee of [9] for suggesting this approach to us. During the revision of this paper, Ramras (see [26, Proposition 4.9]) obtained the following extension of Theorem 5.6 in the case $G=U(n)$ : 
Theorem 5.8. Let $\Sigma$ be a closed, compact, connected, nonorientable surface which is not $\mathbb{R P}^{2}$ (or equivalently, $\chi(\Sigma) \leq 0$ ). Let $P$ be a principal $U(n)$-bundle $(n \geq 2)$ over $\Sigma$, and let $\mathcal{N}_{0}(P)$ be the space of flat connections on $P$. Then $\mathcal{N}_{0}(P)$ is $(1-\chi(\Sigma))(n-1)-1$ connected.

More recent results by Ramras on exact connectivity of $\mathcal{N}_{0}(P)$ can be found in [27, Section 4].

\section{6. $U(n)$-connections on orientable surfaces}

\subsection{Connected components of the representation variety and their reductions}

Any point in

$$
\operatorname{Hom}\left(\Gamma_{\mathbb{R}}\left(\Sigma_{0}^{\ell}\right), U(n)\right) / U(n) \cong X_{\mathrm{YM}}^{\ell, 0}(U(n)) / U(n)
$$

can be represented by $(V, X) \in U(n)^{2 \ell} \times \mathfrak{u}(n)$, where $X$ is a diagonal matrix. Actually, there is a unique representative such that

$$
X=-2 \pi \sqrt{-1}\left(\begin{array}{ccc}
\mu_{1} & & 0 \\
& \ddots & \\
0 & & \mu_{n}
\end{array}\right)
$$

where $\mu_{1} \geq \cdots \geq \mu_{n}$. Suppose that

$$
\mu=\left(\mu_{1}, \ldots, \mu_{n}\right)=(\underbrace{\lambda_{1}, \cdots, \lambda_{1}}_{n_{1}}, \ldots, \underbrace{\lambda_{m}, \ldots, \lambda_{m}}_{n_{m}})
$$

where $\lambda_{1}>\cdots>\lambda_{m}$ and $n_{1}+\cdots+n_{m}=n$. Then

$$
U(n)_{X}=U\left(n_{1}\right) \times \cdots \times U\left(n_{m}\right)
$$

and

$$
\exp (X)=\mathfrak{m}(V) \in S U\left(n_{1}\right) \times \cdots \times S U\left(n_{m}\right),
$$

where $\mathfrak{m}\left(a_{1}, b_{1}, \ldots, a_{\ell}, b_{\ell}\right)=\prod_{i=1}^{\ell}\left[a_{i}, b_{i}\right]$ as before. 
Given an $n_{j} \times n_{j}$ matrix $A_{j}$, let $\operatorname{diag}\left(A_{1}, \ldots, A_{m}\right)$ denote the $n \times n$ matrix

$$
\left(\begin{array}{ccc}
A_{1} & & 0 \\
& \ddots & \\
0 & & A_{m}
\end{array}\right)
$$

where $n=n_{1}+\cdots+n_{m}$. With this notation, we have

$$
\begin{aligned}
X & =-2 \pi \sqrt{-1} \operatorname{diag}\left(\lambda_{1} I_{n_{1}}, \ldots, \lambda_{m} I_{n_{m}}\right), \\
\exp (X) & =\operatorname{diag}\left(e^{-2 \pi \sqrt{-1} \lambda_{1}} I_{n_{1}}, \ldots, e^{-2 \pi \sqrt{-1} \lambda_{m}} I_{n_{m}}\right),
\end{aligned}
$$

where $e^{-2 \pi \sqrt{-1} \lambda_{j}} I_{n_{j}} \in S U\left(n_{j}\right)$, or equivalently, $e^{-2 \pi \sqrt{-1} n_{j} \lambda_{j}}=1$. So

$$
k_{j}=\lambda_{j} n_{j} \in \mathbb{Z}
$$

and

$$
X=-2 \pi \sqrt{-1} \operatorname{diag}\left(\frac{k_{1}}{n_{1}} I_{n_{1}}, \ldots, \frac{k_{m}}{n_{m}} I_{n_{m}}\right) .
$$

For each pair $(n, k) \in \mathbb{Z}_{>0} \times \mathbb{Z}$, define

$$
\begin{aligned}
& I_{n, k}=\left\{\mu=\left(\mu_{1}, \ldots, \mu_{n}\right)=(\underbrace{\frac{k_{1}}{n_{1}}, \ldots, \frac{k_{1}}{n_{1}}}_{n_{1}}, \ldots, \underbrace{\frac{k_{m}}{n_{m}}, \ldots, \frac{k_{m}}{n_{m}}}_{n_{m}}) \mid\right. \\
& \left.n_{j} \in \mathbb{Z}_{>0}, k_{j} \in \mathbb{Z}, \sum_{j=1}^{m} n_{j}=n, \sum_{j=1}^{m} k_{j}=k, \frac{k_{1}}{n_{1}}>\cdots>\frac{k_{m}}{n_{m}}\right\} .
\end{aligned}
$$

Given

$$
\mu=\left(\mu_{1}, \ldots, \mu_{n}\right)=(\underbrace{\frac{k_{1}}{n_{1}}, \ldots, \frac{k_{1}}{n_{1}}}_{n_{1}}, \ldots, \underbrace{\frac{k_{m}}{n_{m}}, \ldots, \frac{k_{m}}{n_{m}}}_{n_{m}}) \in I_{n, k}
$$

let

$$
X_{\mu}=-2 \pi \sqrt{-1} \operatorname{diag}\left(\frac{k_{1}}{n_{1}} I_{n_{1}}, \ldots, \frac{k_{m}}{n_{m}} I_{n_{m}}\right)
$$

and let $C_{\mu}$ be the conjugacy class of $X_{\mu}$. 
Note that if $(V, X) \in X_{\mathrm{YM}}^{\ell, 0}(U(n))$, then $X \in C_{\mu}$ for some $\mu \in \bigcup_{k \in \mathbb{Z}} I_{n, k}$. From now on, we identify $\operatorname{Hom}\left(\Gamma_{\mathbb{R}}\left(\Sigma_{0}^{\ell}\right), U(n)\right)$ with $X_{\mathrm{YM}}^{\ell, 0}(U(n))$.

Given $\mu \in \bigcup_{k \in \mathbb{Z}} I_{n, k}$, define

$X_{\mathrm{YM}}^{\ell, 0}(U(n))_{\mu}=\left\{(V, X) \in U(n)^{2 \ell} \times C_{\mu} \mid V \in\left(U(n)_{X}\right)^{2 \ell}, \mathfrak{m}(V)=\exp (X)\right\}$.

Then

$$
X_{\mathrm{YM}}^{\ell, 0}(U(n))=\bigcup_{k \in \mathbb{Z}} \bigcup_{\mu \in I_{n, k}} X_{\mathrm{YM}}^{\ell, 0}(U(n))_{\mu} .
$$

The $G$-action on $X_{\mathrm{YM}}^{\ell, 0}(U(n))$ preserves $X_{\mathrm{YM}}^{\ell, 0}(U(n))_{\mu}$. We will show that

\section{Proposition 6.1.}

$$
\left\{X_{\mathrm{YM}}^{\ell, 0}(U(n))_{\mu} \mid \mu \in \bigcup_{k \in \mathbb{Z}} I_{n, k}\right\}
$$

are the connected components of $X_{\mathrm{YM}}^{\ell, 0}(U(n))=\operatorname{Hom}\left(\Gamma_{\mathbb{R}}\left(\Sigma_{0}^{\ell}\right), U(n)\right)$. Given

$$
\mu=\left(\mu_{1}, \ldots, \mu_{n}\right)=(\underbrace{\frac{k_{1}}{n_{1}}, \ldots, \frac{k_{1}}{n_{1}}}_{n_{1}}, \ldots, \underbrace{\frac{k_{m}}{n_{m}}, \ldots, \frac{k_{m}}{n_{m}}}_{n_{m}}) \in I_{n, k},
$$

we have a homeomorphism

$$
X_{\mathrm{YM}}^{\ell, 0}(U(n))_{\mu} / U(n)=\prod_{j=1}^{m} X_{\mathrm{YM}}^{\ell, 0}\left(U\left(n_{j}\right)\right)_{\frac{k_{j}}{n_{j}}, \ldots, \frac{k_{j}}{n_{j}}} / U\left(n_{j}\right)
$$

and a homotopy equivalence of homotopic orbit spaces

$$
X_{\mathrm{YM}}^{\ell, 0}(U(n))_{\mu}^{h U(n)}=\prod_{j=1}^{m}\left(X_{\mathrm{YM}}^{\ell, 0}\left(U\left(n_{j}\right)\right)_{\frac{k_{j}}{n_{j}}, \ldots, \frac{k_{j}}{n_{j}}}\right)^{h U\left(n_{j}\right)} .
$$

Proof. Let $\mu$ be as in (6.3). Let $\pi: X_{\mathrm{YM}}^{\ell, 0}(U(n)) \rightarrow C_{\mu}$ be defined by $(V, X) \mapsto$ $X$. Then $\pi$ is a fibration, so there is a homeomorphism

$$
X_{\mathrm{YM}}^{\ell, 0}(U(n))_{\mu} / U(n)=\pi^{-1}\left(X_{\mu}\right) / U(n)_{X_{\mu}}
$$


and a homotopy equivalence

$$
X_{\mathrm{YM}}^{\ell, 0}(U(n))_{\mu}^{h U(n)}=\pi^{-1}\left(X_{\mu}\right)^{h U(n)_{X_{\mu}}},
$$

where $U(n)_{X_{\mu}}=U\left(n_{1}\right) \times \cdots \times U\left(n_{m}\right)$. We have

$$
\begin{aligned}
\pi^{-1}\left(X_{\mu}\right) & \cong\left\{V \in U(n)_{X_{\mu}}^{2 \ell} \mid \mathfrak{m}(V)=\exp \left(X_{\mu}\right)\right\} \\
& \cong \prod_{j=1}^{m}\left\{V \in U\left(n_{j}\right)^{2 \ell} \mid \mathfrak{m}(V)=e^{-2 \pi \sqrt{-1} k_{j} / n_{j}} I_{n_{j}}\right\} \\
& =\prod_{j=1}^{m} X_{\mathrm{YM}}^{\ell, 0}\left(U\left(n_{j}\right)\right)_{\frac{k_{j}}{n_{j}}, \ldots, \frac{k_{j}}{n_{j}}}
\end{aligned}
$$

This proves (6.4). The set

$$
X_{\mathrm{YM}}^{\ell, 0}\left(U\left(n_{j}\right)\right)_{\frac{k_{j}}{n_{j}}, \ldots, \frac{k_{j}}{n_{j}}}=\left\{V \in U\left(n_{j}\right)^{2 \ell} \mid \mathfrak{m}(V)=e^{-2 \pi \sqrt{-1} k_{j} / n_{j}} I_{n_{j}}\right\}
$$

is nonempty and connected by [11, Theorem 3]. So $\pi^{-1}\left(X_{\mu}\right)$ is nonempty and connected. Together with $(6.5)$, this implies that $X_{\mathrm{YM}}^{\ell, 0}(U(n))_{\mu}$ is nonempty and connected.

Define $T: X_{\mathrm{YM}}^{\ell, 0}(U(n)) \rightarrow \mathbb{R}^{n}$ by

$$
T(V, X)=\left(\frac{\sqrt{-1}}{2 \pi} \operatorname{Tr} X,\left(\frac{\sqrt{-1}}{2 \pi}\right)^{2} \operatorname{Tr}\left(X^{2}\right), \ldots,\left(\frac{\sqrt{-1}}{2 \pi}\right)^{n} \operatorname{Tr}\left(X^{n}\right)\right)
$$

where $V \in U(n)^{2 \ell}$ and $X \in \mathfrak{u}(n)$. The characteristic polynomial

$$
P_{X}(t)=\operatorname{det}(t I-X)=\left(t+2 \pi \sqrt{-1} \mu_{1}\right) \cdots\left(t+2 \pi \sqrt{-1} \mu_{n}\right)
$$

of a matrix $X$ is determined by $\operatorname{Tr} X, \operatorname{Tr}\left(X^{2}\right), \ldots, \operatorname{Tr}\left(X^{n}\right)$, and the conjugacy class of $X$ in $\mathfrak{u}(n)$ is determined by $P_{X}(t)$, so $T(V, X)=T\left(V^{\prime}, X^{\prime}\right)$ if and only if $X$ and $X^{\prime}$ are in the same conjugacy class.

Given $\mu \in \bigcup_{k \in \mathbb{Z}} I_{n, k}$, define

$$
v_{\mu}=\left(\sum_{i=1}^{n} \mu_{i}, \sum_{i=1}^{n} \mu_{i}^{2}, \ldots, \sum_{i=1}^{n} \mu_{i}^{n}\right) \in \mathbb{Q}^{n} .
$$

Note that $v_{\mu}=v_{\mu^{\prime}}$ if and only if $\mu=\mu^{\prime}$. 
The function $T$ is a continuous function, and its image $\left\{v_{\mu} \mid \mu \in \bigcup_{k \in \mathbb{Z}}\right.$ $\left.I_{n, k}\right\}$ is a discrete set, so

$$
\left\{X_{\mathrm{YM}}^{\ell, 0}(U(n))_{\mu}=T^{-1}\left(v_{\mu}\right) \mid \mu \in \bigcup_{k \in \mathbb{Z}} I_{n, k}\right\}
$$

are connected components of $X_{\mathrm{YM}}^{\ell, 0}(U(n))$.

\subsection{Equivariant Morse stratification and equivariant Poincaré series}

Let $P^{n, k}$ be the topological principal $U(n)$-bundle on $\Sigma_{0}^{\ell}$ with $c_{1}\left(P^{n, k}\right)=$ $k[\omega] \in H^{2}\left(\Sigma_{0}^{\ell}\right)$. Let $\mathcal{A}^{n, k}$ be the space of $U(n)$-connections on $P^{n, k}$, and let $\mathcal{N}^{n, k} \subset \mathcal{A}^{n, k}$ be the space of Yang-Mills $U(n)$-connections on $P^{n, k}$. Let $\mathcal{G}^{n, k}$ be the group of gauge transformations on $P^{n, k}$, and let $\mathcal{G}_{0}^{n, k}$ be the subgroup of gauge transformations which take value of the identity $e$ at a fixed point $x_{0} \in \Sigma_{0}^{\ell}$. We have

$$
\operatorname{Hom}\left(\Gamma_{\mathbb{R}}\left(\Sigma_{0}^{\ell}\right), U(n)\right)=\bigcup_{k \in \mathbb{Z}} \operatorname{Hom}\left(\Gamma_{\mathbb{R}}\left(\Sigma_{0}^{\ell}\right), U(n)\right)_{k}
$$

where $\operatorname{Hom}\left(\Gamma_{\mathbb{R}}\left(\Sigma_{0}^{\ell}\right), U(n)\right)_{k} \cong \mathcal{N}^{n, k} / \mathcal{G}_{0}^{n, k}$. The connected components of $\operatorname{Hom}\left(\Gamma_{\mathbb{R}}\left(\Sigma_{0}^{\ell}\right), U(n)\right)_{k}$ are

$$
\left\{X_{\mathrm{YM}}^{\ell, 0}(U(n))_{\mu} \mid \mu \in I_{n, k}\right\}
$$

Let $\mathcal{N}_{\mu}$ be the preimage of $X_{\mathrm{YM}}^{\ell, 0}(U(n))_{\mu}$ under the projection

$$
\mathcal{N}^{n, k} \rightarrow \mathcal{N}^{n, k} / \mathcal{G}_{0}^{n, k} \cong \operatorname{Hom}\left(\Gamma_{\mathbb{R}}\left(\Sigma_{0}^{\ell}\right), U(n)\right)_{k}
$$

so that $X_{\mathrm{YM}}^{\ell, 0}(U(n))_{\mu}=\mathcal{N}_{\mu} / \mathcal{G}_{0}(P)$.

We fix $(n, k) \in \mathbb{Z}_{>0} \times \mathbb{Z}$, and write $\mathcal{G}=\mathcal{G}^{n, k}$. Let $\mathcal{A}_{\mu}$ be the stable manifold of $\mathcal{N}_{\mu}$ with respect to Yang-Mills functional. Then

$$
\mathcal{A}^{n, k}=\bigcup_{\mu \in I_{n, k}} \mathcal{A}_{\mu}
$$

is the $\mathcal{G}$-equivariant Morse stratification of $\mathcal{A}^{n, k}$ given by the Yang-Mills functional $[1,6,25,30]$. Let $\lambda_{\mu}$ be the real codimension of $\mathcal{A}_{\mu}$ in $\mathcal{A}^{n, k}$. It was 
computed in $[1$, Section 7$]$ that

$$
\lambda_{\mu}=2 d_{\mu}, \quad d_{\mu}=\sum_{i<j}\left(\mu_{i}-\mu_{j}+(\ell-1)\right) .
$$

The gradient flow of the Yang-Mills functional gives a $\mathcal{G}$-equivariant deformation retraction $\mathcal{A}_{\mu} \rightarrow \mathcal{N}_{\mu}$. For the purpose of equivariant cohomology, the following equivariant pairs are equivalent:

$$
\left(\mathcal{A}_{\mu}, \mathcal{G}\right) \sim\left(\mathcal{N}_{\mu}, \mathcal{G}\right) \sim\left(X_{\mathrm{YM}}^{\ell, 0}(U(n))_{\mu}, U(n)\right)
$$

In other words, we have the following homotopy equivalences of homotopic orbit spaces:

$$
\mathcal{A}_{\mu}{ }^{h \mathcal{G}} \sim \mathcal{N}_{\mu}{ }^{h \mathcal{G}} \sim X_{\mathrm{YM}}^{\ell, 0}(U(n))_{\mu}{ }^{h U(n)}
$$

Together with the reduction Proposition 6.1, we conclude that

Theorem 6.2. Let $K$ be a field. For any

$$
\mu=\left(\mu_{1}, \ldots, \mu_{n}\right)=(\underbrace{\frac{k_{1}}{n_{1}}, \ldots, \frac{k_{1}}{n_{1}}}_{n_{1}}, \ldots, \underbrace{\frac{k_{m}}{n_{m}}, \ldots, \frac{k_{m}}{n_{m}}}_{n_{m}}) \in I_{n, k}
$$

we have

$$
\begin{aligned}
H_{\mathcal{G}}^{*}\left(\mathcal{A}_{\mu} ; K\right) & =H_{\mathcal{G}}^{*}\left(\mathcal{N}_{\mu} ; K\right)=H_{U(n)}^{*}\left(X_{\mathrm{YM}}^{\ell, 0}(U(n))_{\mu} ; K\right) \\
& \cong \bigotimes_{j=1}^{m} H_{U\left(n_{j}\right)}^{*}\left(V_{s s}\left(P^{n_{j}, k_{j}}\right) ; K\right) \\
P_{t}^{\mathcal{G}}\left(\mathcal{A}_{\mu} ; K\right) & =P_{t}^{\mathcal{G}}\left(\mathcal{N}_{\mu} ; K\right)=P_{t}^{U(n)}\left(X_{\mathrm{YM}}^{\ell, 0}(U(n))_{\mu} ; K\right) \\
& =\prod_{i=1}^{m} P_{t}^{U\left(n_{j}\right)}\left(V_{s s}\left(P^{n_{j}, k_{j}}\right) ; K\right)
\end{aligned}
$$




\subsection{Involution}

Given $\mu \in I_{n, k}$, define

$$
\begin{gathered}
Z_{\mathrm{YM}}^{\ell, 1}(U(n))_{\mu}=\left\{(V, c, \bar{V}, \bar{c}, X) \in U(n)^{2(2 \ell+1)} \times C_{\mu} \mid V, c \bar{V} c^{-1} \in\left(U(n)_{X}\right)^{2 \ell},\right. \\
\mathfrak{m}(V)=\exp (X / 2) c \bar{c}, \mathfrak{m}(\bar{V})=\bar{c} \exp (-X / 2) c\} \\
Z_{\mathrm{YM}}^{\ell, 2}(U(n))_{\mu}=\left\{(V, d, c, \bar{V}, \bar{d}, \bar{c}, X) \in U(n)^{2(2 \ell+2)} \times C_{\mu} \mid d^{-1}, c \bar{c} \in U(n)_{X},\right. \\
V, d^{-1} c \bar{V} c^{-1} d \in\left(U(n)_{X}\right)^{2 \ell}, \mathfrak{m}(V)=\exp (X / 2) c \bar{d} c^{-1} d, \\
\left.\mathfrak{m}(\bar{V})=\bar{c} d \exp (-X / 2) \bar{c}^{-1} \bar{d}\right\} .
\end{gathered}
$$

Then for $i=1,2$,

$$
\begin{gathered}
Z_{\mathrm{YM}}^{\ell, i}(U(n))=\bigcup_{k \in \mathbb{Z}} \bigcup_{\mu \in I_{n, k}} Z_{\mathrm{YM}}^{\ell, i}(U(n))_{\mu}, \\
\Phi_{U(n)}^{\ell, i}\left(Z_{\mathrm{YM}}^{\ell, i}(U(n))_{\mu}\right)=X_{\mathrm{YM}}^{2 \ell+i-1,0}(U(n))_{\mu} .
\end{gathered}
$$

Define $\tau_{0}: I_{n, k} \rightarrow I_{n,-k}$ by

$$
\left(\mu_{1}, \mu_{2}, \ldots, \mu_{n}\right) \longmapsto\left(-\mu_{n}, \ldots,-\mu_{2},-\mu_{1}\right)
$$

It is easy to check that if $X \in C_{\mu}$, then $-\operatorname{Ad}(\bar{c})(X) \in C_{\tau_{0}(\mu)}$. So

$$
\tau\left(Z_{\mathrm{YM}}^{\ell, i}(U(n))_{\mu}\right)=Z_{\mathrm{YM}}^{\ell, i}(U(n))_{\tau_{0}(\mu)} .
$$

Thus we conclude that:

Theorem 6.3. The set

$$
Z_{\mathrm{YM}}^{\ell, i}(U(n))_{\mu}^{\tau}=Z_{\mathrm{YM}}^{\ell, i}(U(n))^{\tau} \cap Z_{\mathrm{YM}}^{\ell, i}(U(n))_{\mu}
$$

is nonempty if and only if $X_{\mu}$ is conjugate to $-X_{\mu}$, i.e., $\tau_{0}(\mu)=\mu$. In other words, if we define

$$
I_{n}=I_{n, 0}^{\tau_{0}}=\left\{\mu \in I_{n, 0} \mid \tau_{0}(\mu)=\mu\right\}
$$

Then

$$
Z_{\mathrm{YM}}^{\ell, i}(U(n))^{\tau}=\bigcup_{\mu \in I_{n}} Z_{\mathrm{YM}}^{\ell, i}(U(n))_{\mu}^{\tau}
$$




\section{7. $U(n)$-connections on nonorientable surfaces}

\subsection{Connected components of the representation variety and their reductions}

Given $\mu \in I_{n, k}$, let $C_{\mu / 2}$ denote the conjugacy class of $X_{\mu} / 2$ in $\mathfrak{u}(n)$. $Z_{\mathrm{YM}}^{\ell, 1}(U(n))_{\mu}^{\tau}$ can be identified with

$$
\begin{gathered}
X_{\mathrm{YM}}^{\ell, 1}(U(n))_{\mu}=\left\{(V, c, X) \in U(n)^{2 \ell+1} \times C_{\mu / 2} \mid V \in\left(U(n)_{X}\right)^{2 \ell},\right. \\
\left.\operatorname{Ad}(c)(X)=-X, \mathfrak{m}(V)=\exp (X) c^{2}\right\},
\end{gathered}
$$

while $Z_{\mathrm{YM}}^{\ell, 2}(U(n))_{\mu}^{\tau}$ can be identified with

$$
\begin{gathered}
X_{\mathrm{YM}}^{\ell, 2}(U(n))_{\mu}=\left\{(V, d, c, X) \in U(n)^{2 \ell+2} \times C_{\mu / 2} \mid(V, d) \in\left(U(n)_{X}\right)^{2 \ell+1},\right. \\
\left.\operatorname{Ad}(c)(X)=-X, \mathfrak{m}(V)=\exp (X) c d c^{-1} d\right\} .
\end{gathered}
$$

Note that a Yang-Mills connection on a principal $U(n)$-bundle $P$ over a nonorientable surface $\Sigma$ induces a flat connection on the $U(1)$-bundle $\operatorname{det}(P)$. More explicitly, define

$$
\begin{aligned}
\operatorname{det}: U(n)^{2 \ell} & \longrightarrow U(1)^{2 \ell}, \\
\left(a_{1}, b_{1}, \ldots, a_{\ell}, b_{\ell}\right) & \longmapsto\left(\operatorname{det}\left(a_{1}\right), \operatorname{det}\left(b_{1}\right), \ldots, \operatorname{det}\left(a_{\ell}\right), \operatorname{det}\left(b_{\ell}\right)\right) \in U(1)^{2 \ell} .
\end{aligned}
$$

We have

$$
\begin{aligned}
& \operatorname{det}: X_{\mathrm{YM}}^{\ell, 1}(U(n)) \rightarrow X_{\text {flat }}^{\ell, 1}(U(1)) \quad(V, c, X) \longmapsto(\operatorname{det}(V), \operatorname{det}(c)), \\
& \operatorname{det}: X_{\mathrm{YM}}^{\ell, 2}(U(n)) \rightarrow X_{\text {flat }}^{\ell, 2}(U(1)) \quad(V, d, c, X) \longmapsto(\operatorname{det}(V), \operatorname{det}(d), \operatorname{det}(c)),
\end{aligned}
$$

where $X_{\text {flat }}^{\ell, 1}(U(1))$ and $X_{\text {flat }}^{\ell, 2}(U(1))$ are as in Example 2.1. Let

$$
\begin{aligned}
& X_{\text {flat }}^{\ell, 1}(U(1))^{ \pm 1}=\left\{\left(a_{1}, b_{1}, \ldots, a_{\ell}, b_{\ell}, c\right) \in U(1)^{2 \ell+1} \mid c= \pm 1\right\}, \\
& X_{\text {flat }}^{\ell, 2}(U(1))^{ \pm 1}=\left\{\left(a_{1}, b_{1}, \ldots, a_{\ell}, b_{\ell}, d, c\right) \in U(1)^{2 \ell+2} \mid d= \pm 1\right\} .
\end{aligned}
$$

Then $X_{\text {flat }}^{\ell, i}(U(1))^{+1}$ and $X_{\text {flat }}^{\ell, i}(U(1))^{-1}$ are the two connected components of $X_{\text {flat }}^{\ell, i}(U(1))$. Let

$$
\begin{aligned}
& X_{\mathrm{YM}}^{\ell, i}(U(n))^{ \pm 1}=(\operatorname{det})^{-1}\left(X_{\text {flat }}^{\ell, i}(U(1))^{ \pm}\right), \\
& X_{\mathrm{YM}}^{\ell, i}(U(n))_{\mu}^{ \pm 1}=X_{\mathrm{YM}}^{\ell, i}(U(n))_{\mu} \cap X_{\mathrm{YM}}^{\ell, i}(U(n))^{ \pm} .
\end{aligned}
$$


Then

$$
X_{\mathrm{YM}}^{\ell, i}(U(n))_{\mu}=X_{\mathrm{YM}}^{\ell, i}(U(n))_{\mu}^{+1} \cup X_{\mathrm{YM}}^{\ell, i}(U(n))_{\mu}^{-1} .
$$

Any $\mu \in I_{n}$ is of the following form:

$$
\mu=(\nu, \underbrace{0, \ldots, 0}_{n_{0}}, \tau_{0}(\nu))
$$

where $\nu \in I_{n^{\prime}, k}, \tau_{0}(\nu) \in I_{n^{\prime},-k}, n^{\prime} \geq 0, n_{0} \geq 0, k>0$.

We will show that

Proposition 7.1. Let $\mu=(\nu, \underbrace{0, \ldots, 0}_{n_{0}}, \tau_{0}(\nu)) \in I_{n}=I_{n, 0}^{\tau_{0}}$, where

$$
\nu \in I_{n^{\prime}, k}, \quad n^{\prime}, n_{0} \geq 0, \quad 2 n^{\prime}+n_{0}=n, \quad k>0 .
$$

(i) Suppose that $n_{0}>0$. For $i=1,2, X_{\mathrm{YM}}^{\ell, i}(U(n))_{\mu}^{+1}, X_{\mathrm{YM}}^{\ell, i}(U(n))_{\mu}^{-1}$ are nonempty and connected for $\ell \geq 1$.

(ii) Suppose that $n_{0}=0$ so that $2 n^{\prime}=n>0$. For $i=1,2$,

$$
X_{\mathrm{YM}}^{\ell, i}(U(n))_{\mu}=X_{\mathrm{YM}}^{\ell, i}(U(n))_{\mu}^{(-1)^{n^{\prime} i+k}} .
$$

$X_{\mathrm{YM}}^{\ell, i}(U(n))_{\mu}$ is nonempty and connected unless $i=1$ and $\ell=0$.

Proof. (i) $n_{0}>0 . \nu$ is of the form

$$
\nu=(\underbrace{\frac{k_{1}}{n_{1}}, \ldots \frac{k_{1}}{n_{1}}}_{n_{1}}, \ldots, \underbrace{\frac{k_{m}}{n_{m}}, \ldots, \frac{k_{m}}{n_{m}}}_{n_{m}})
$$

where

$$
\frac{k_{1}}{n_{1}}>\cdots>\frac{k_{m}}{n_{m}}>0
$$

and

$$
X_{\mu}=-2 \pi \sqrt{-1} \operatorname{diag}\left(\frac{k_{1}}{n_{1}} I_{n_{1}}, \ldots, \frac{k_{m}}{n_{m}} I_{n_{m}}, 0 I_{n_{0}},-\frac{k_{m}}{n_{m}} I_{n_{m}}, \ldots,-\frac{k_{1}}{n_{1}} I_{n_{1}}\right) .
$$

Let $\pi_{1}: X_{\mathrm{YM}}^{\ell, 1}(U(n))_{\mu} \rightarrow C_{\mu / 2}$ be defined by $(V, c, X) \mapsto X$, and let $\pi_{2}$ : $X_{\mathrm{YM}}^{\ell, 2}(U(n))_{\mu} \rightarrow C_{\mu / 2}$ be defined by $(V, d, c, X) \mapsto X$. Then $\pi_{1}$ and $\pi_{2}$ are 
fibrations. So

$$
X_{\mathrm{YM}}^{\ell, i}(U(n))_{\mu} / U(n) \cong \pi_{i}^{-1}\left(X_{\mu} / 2\right) / U(n)_{\mu},
$$

where

$$
U(n)_{\mu}=U(n)_{X_{\mu} / 2}=U(n)_{X_{\mu}} .
$$

Let

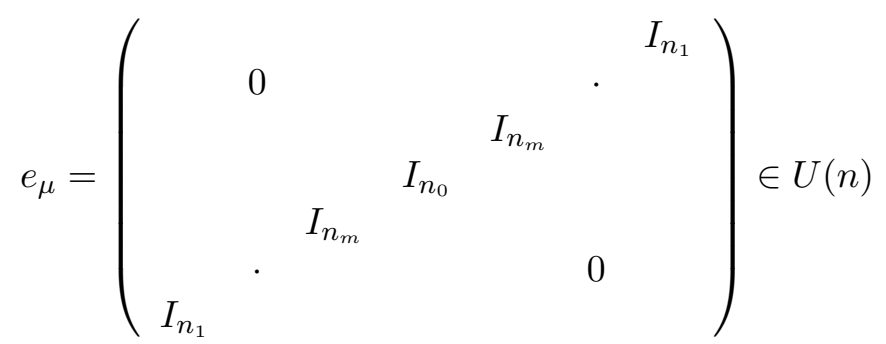

Then

$$
\operatorname{Ad}\left(e_{\mu}\right) X_{\mu}=-X_{\mu}, \quad \operatorname{det}\left(e_{\mu}\right)=(-1)^{n^{\prime}}, \quad e_{\mu}^{2}=I_{n}
$$

We have $\pi_{i}^{-1}\left(X_{\mu} / 2\right) \cong V_{\mu}^{i}$, where

$$
\begin{aligned}
& V_{\mu}^{1}=\left\{\left(V, c^{\prime}\right) \in U(n)_{\mu}^{2 \ell+1} \mid \mathfrak{m}(V)=\exp \left(X_{\mu} / 2\right) e_{\mu} c^{\prime} e_{\mu} c^{\prime}\right\} \\
& V_{\mu}^{2}=\left\{\left(V, d, c^{\prime}\right) \in U(n)_{\mu}^{2 \ell+2} \mid \mathfrak{m}(V)=\exp \left(X_{\mu} / 2\right) e_{\mu} c^{\prime} d\left(e_{\mu} c^{\prime}\right)^{-1} d\right\}
\end{aligned}
$$

Under the identification $\pi_{i}^{-1}\left(X_{\mu} / 2\right) \cong V_{\mu}^{i}$, the actions of $U(n)_{\mu}$ on $V_{\mu}^{1}$ and $V_{\mu}^{2}$ are given by

$$
\begin{aligned}
g \cdot\left(V, c^{\prime}\right) & =\left(g V g^{-1},\left(e_{\mu} g e_{\mu}\right) c^{\prime} g^{-1}\right) \quad \text { and } \\
g \cdot\left(V, d, c^{\prime}\right) & =\left(g V g^{-1}, g d g^{-1},\left(e_{\mu} g e_{\mu}\right) c^{\prime} g^{-1}\right)
\end{aligned}
$$

respectively, where $V \in U(n)_{\mu}^{2 \ell}$, and $g, c^{\prime}, d \in U(n)_{\mu}$.

Any $a_{i}, b_{i}, c^{\prime}, d \in U(n)_{\mu}$ are of the form

$$
\begin{aligned}
a_{i} & =\operatorname{diag}\left(A_{1}^{i}, \ldots, A_{m}^{i}, A^{i}, \bar{A}_{m}^{i}, \ldots, \bar{A}_{1}^{i}\right), \\
b_{i} & =\operatorname{diag}\left(B_{1}^{i}, \ldots, B_{m}^{i}, B^{i}, \bar{B}_{m}^{i}, \ldots, \bar{B}_{1}^{i}\right), \\
c^{\prime} & =\operatorname{diag}\left(\bar{C}_{1}, \ldots \bar{C}_{m}, C, C_{m}, \ldots, C_{1}\right), \\
d & =\operatorname{diag}\left(D_{1}, \ldots D_{m}, D, \bar{D}_{m}, \ldots, \bar{D}_{1}\right),
\end{aligned}
$$

where

$$
A_{j}^{i}, \bar{A}_{j}^{i}, B_{j}^{i}, \bar{B}_{j}^{i}, C_{j}, \bar{C}_{j}, D_{j}, \bar{D}_{j} \in U\left(n_{j}\right), \quad A^{i}, B^{i}, C, D \in U\left(n_{0}\right) .
$$


If $\left(V, c^{\prime}\right) \in V_{\mu}^{1}$, we have

$$
\prod_{i=1}^{\ell}\left[A_{j}^{i}, B_{j}^{i}\right]=e^{\frac{-\pi \sqrt{-1} k_{j}}{n_{j}}} C_{j} \bar{C}_{j}, \quad \prod_{i=1}^{\ell}\left[\bar{A}_{j}^{i}, \bar{B}_{j}^{i}\right]=e^{\frac{\pi \sqrt{-1} k_{j}}{n_{j}}} \bar{C}_{j} C_{j}
$$

for $j=1, \ldots, m$, and

$$
\prod_{i=1}^{\ell}\left[A^{i}, B^{i}\right]=C^{2}
$$

By (7.2), we have

$$
1=\operatorname{det}\left(e^{\frac{-\pi \sqrt{-1} k_{j}}{n_{j}}} C_{j} \bar{C}_{j}\right)=(-1)^{k_{j}} \operatorname{det}\left(C_{j}\right) \operatorname{det}\left(\bar{C}_{j}\right)
$$

By (7.3), we have $1=\operatorname{det}\left(C^{2}\right)=\operatorname{det}(C)^{2}$. Recall that $\operatorname{det}(c)=\operatorname{det}\left(e_{\mu}\right)$ $\operatorname{det}\left(c^{\prime}\right)=(-1)^{n^{\prime}} \operatorname{det}\left(c^{\prime}\right)$, so

$$
\operatorname{det}(c)=(-1)^{n^{\prime}+k} \operatorname{det}(C), \quad \operatorname{det}(C)= \pm 1
$$

Note that the equations in (7.2) are exactly the defining equations for

$$
Z_{\mathrm{YM}}^{\ell, 1}\left(U\left(n_{j}\right)\right)_{\frac{k_{j}}{n_{j}}, \ldots, \frac{k_{j}}{n_{j}}}
$$

where $Z_{\mathrm{YM}}^{\ell, 1}(U(n))_{\mu}$ is defined as in Section 6.3, and (7.3) is exactly the defining equation for $X_{\text {flat }}^{\ell, 1}\left(U\left(n_{0}\right)\right)$. So we have the following homeomorphism:

$$
V_{\mu}^{1} \cong X_{\text {flat }}^{\ell, 1}\left(U\left(n_{0}\right)\right) \times \prod_{j=1}^{m} Z_{\mathrm{YM}}^{\ell, 1}\left(U\left(n_{j}\right)\right)_{\frac{k_{j}}{n_{j}}, \ldots, \frac{k_{j}}{n_{j}}} .
$$

If $\left(V, d, c^{\prime}\right) \in V_{\mu}^{2}$, we have

$$
\begin{aligned}
& \prod_{i=1}^{\ell}\left[A_{j}^{i}, B_{j}^{i}\right]=e^{\frac{-\pi \sqrt{-1} k_{j}}{n_{j}}} C_{j} \bar{D}_{j} C_{j}^{-1} D_{j}, \\
& \prod_{i=1}^{\ell}\left[\bar{A}_{j}^{i}, \bar{B}_{j}^{i}\right]=e^{\frac{\pi \sqrt{-1} k_{j}}{n_{j}}} \bar{C}_{j} D_{j} \bar{C}_{j}^{-1} \bar{D}_{j}
\end{aligned}
$$


for $j=1, \ldots, m$, and

$$
\prod_{i=1}^{\ell}\left[A^{i}, B^{i}\right]=C D C^{-1} D
$$

By (7.6), we have

$$
1=\operatorname{det}\left(e^{\frac{-\pi \sqrt{-1} k_{j}}{n_{j}}} D_{j} \bar{D}_{j}\right)=(-1)^{k_{j}} \operatorname{det}\left(D_{j}\right) \operatorname{det}\left(\bar{D}_{j}\right) .
$$

By (7.7), we have $1=\operatorname{det}\left(D^{2}\right)=\operatorname{det}(D)^{2}$. We conclude that

$$
\operatorname{det}(d)=(-1)^{k} \operatorname{det}(D), \quad \operatorname{det}(D)= \pm 1 .
$$

Note that the equations in (7.6) are exactly the defining equations for

$$
Z_{\mathrm{YM}}^{\ell, 2}\left(U\left(n_{j}\right)\right)_{\frac{k_{j}}{n_{j}}, \ldots, \frac{k_{j}}{n_{j}}},
$$

where $Z_{\mathrm{YM}}^{\ell, 2}(U(n))_{\mu}$ is defined as in Section 6.3, and (7.7) is exactly the defining equation for $X_{\text {flat }}^{\ell, 2}\left(U\left(n_{0}\right)\right)$. So we have the following homeomorphism:

$$
V_{\mu}^{2} \cong X_{\text {flat }}^{\ell, 2}\left(U\left(n_{0}\right)\right) \times \prod_{j=1}^{m} Z_{\mathrm{YM}}^{\ell, 2}\left(U\left(n_{j}\right)\right)_{\frac{k_{j}}{n_{j}}, \ldots, \frac{k_{j}}{n_{j}}} .
$$

The homeomorphisms $(7.5),(7.9)$ are $U(n)_{\mu}$-equivariant: for $i=1,2$, it is straightforward to check that the action of

$$
U(n)_{\mu}=U\left(n_{1}\right) \times \cdots \times U\left(n_{m}\right) \times U\left(n_{0}\right) \times U\left(n_{m}\right) \times \cdots \times U\left(n_{1}\right)
$$

on $V_{\mu}^{i}$ is compatible with the action of $U\left(n_{0}\right)$ on $X_{\text {flat }}^{\ell, i}\left(U\left(n_{0}\right)\right)$ and the actions of $U\left(n_{j}\right)^{2}$ on $Z_{\mathrm{YM}}^{\ell, i}\left(U\left(n_{j}\right)\right)_{\frac{k_{j}}{n_{j}}, \ldots, \frac{k_{j}}{n_{j}}}$. So

$$
\begin{aligned}
& X_{\mathrm{YM}}^{\ell, i}(U(n))_{\mu} / U(n) \\
& \cong V_{\mu}^{i} / U(n)_{\mu} \\
& \cong X_{\text {flat }}^{\ell, i}\left(U\left(n_{0}\right)\right) / U\left(n_{0}\right) \times \prod_{j=1}^{m}\left(Z_{\mathrm{YM}}^{\ell, i}\left(U\left(n_{j}\right)\right)_{\left.\frac{k_{j}}{n_{j}}, \ldots, \frac{k_{j}}{n_{j}} / U\left(n_{j}\right)^{2}\right)}\right. \\
& \cong X_{\text {flat }}^{\ell, i}\left(U\left(n_{0}\right)\right) / U\left(n_{0}\right) \times \prod_{j=1}^{m}\left(X_{\mathrm{YM}}^{2 \ell+i-1,0}\left(U\left(n_{j}\right)\right)_{\frac{k_{j}}{n_{j}}, \ldots, \frac{k_{j}}{n_{j}}} / U\left(n_{j}\right)\right) \\
& \cong X_{\text {flat }}^{\ell, i}\left(U\left(n_{0}\right)\right) / U\left(n_{0}\right) \times X_{\mathrm{YM}}^{2 \ell+i-1,0}\left(U\left(n^{\prime}\right)\right)_{\nu} / U\left(n^{\prime}\right) .
\end{aligned}
$$


From (7.4) and (7.8), we see that

$$
\begin{aligned}
& X_{\mathrm{YM}}^{\ell, i}(U(n))_{\mu}^{ \pm 1} / U(n) \\
& \quad \cong\left(X_{\text {flat }}^{\ell, i}\left(U\left(n_{0}\right)\right)^{ \pm(-1)^{n^{\prime} i+k}}\right) / U\left(n_{0}\right) \times X_{\mathrm{YM}}^{2 \ell+i-1,0}\left(U\left(n^{\prime}\right)\right)_{\nu} / U\left(n^{\prime}\right)
\end{aligned}
$$

where

$$
X_{\text {flat }}^{\ell, i}\left(U\left(n_{0}\right)\right)^{ \pm 1}=X_{\mathrm{YM}}^{\ell, i}\left(U\left(n_{0}\right)\right)_{0, \ldots, 0}^{ \pm 1} .
$$

Recall from Proposition 6.1 that if $2 \ell+i-1 \geq 1$, then

$$
X_{\mathrm{YM}}^{2 \ell+i-1,0}\left(U\left(n^{\prime}\right)\right)_{\nu}
$$

is nonempty and connected. By Theorem 5.6,

$$
X_{\text {flat }}^{\ell, i}\left(U\left(n_{0}\right)\right)^{+1}, \quad X_{\text {flat }}^{\ell, i}\left(U\left(n_{0}\right)\right)^{-1}
$$

are nonempty and connected for $\ell \geq 1$. We conclude that $X_{\mathrm{YM}}^{\ell, i}(U(n))_{\mu}^{+1}$ and $X_{\mathrm{YM}}^{\ell, i}(U(n))_{\mu}^{-1}$ are nonempty and connected for $\ell \geq 1$.

(ii) $n_{0}=0$. The calculations in this case are the same as those in (i), except that we do not have the factor $U\left(n_{0}\right)$ so we do not have the matrices $A^{i}, B^{i}, C, D$. We conclude that $\operatorname{det}(c)=(-1)^{n^{\prime}+k}$ when $i=1$ and $\operatorname{det}(d)=$ $(-1)^{k}$ when $i=2$. So

$$
X_{\mathrm{YM}}^{\ell, i}(U(n))_{\mu}=X_{\mathrm{YM}}^{\ell, i}(U(n))_{\mu}^{(-1)^{n^{\prime} i+k}}
$$

and

$$
X_{\mathrm{YM}}^{\ell, i}(U(n))_{\mu} / U(n) \cong X_{\mathrm{YM}}^{2 \ell+i-1,0}\left(U\left(n^{\prime}\right)\right)_{\nu} / U\left(n^{\prime}\right) .
$$

Recall that $X_{\mathrm{YM}}^{2 \ell+i-1,0}\left(U\left(n^{\prime}\right)\right)_{\nu}$ is nonempty and connected if $2 \ell+i-1 \geq 1$. So for $i=1,2, X_{\mathrm{YM}}^{\ell, i}(U(n))_{\mu}$ is nonempty and connected unless $i=1$ and $\ell=0$.

Let $I_{n}=I_{n, 0}^{\tau_{0}}$. Then $I_{n}=I_{n}^{0} \cup I_{n}^{i,+} \cup I_{n}^{i,-}$, where

$$
\begin{aligned}
I_{n}^{0} & =\left\{\mu \in I_{n}, \mu_{i}=0 \text { for some } i\right\} \\
I_{n}^{i,+} & =\left\{\mu=\left(\nu, \tau_{0}(\nu)\right) \mid \nu \in I_{n^{\prime}, k}, n^{\prime} i+k \text { is even }\right\} \\
I_{n}^{i,-} & =\left\{\mu=\left(\nu, \tau_{0}(\nu)\right) \mid \nu \in I_{n^{\prime}, k}, n^{\prime} i+k \text { is odd }\right\}
\end{aligned}
$$

where $i=1,2$. When $n$ is odd, we have $I_{n}=I_{n}^{0}$.

The proof of Proposition 7.1 gives the following. 
Proposition 7.2. Suppose that $i=1,2$ and $\ell \geq 1$.

(i) If $n$ is odd, the connected components of $X_{\mathrm{YM}}^{\ell, i}(U(n))=\operatorname{Hom}\left(\Gamma_{\mathbb{R}}\left(\Sigma_{i}^{\ell}\right)\right.$, $U(n))$ are

$$
\left\{X_{\mathrm{YM}}^{\ell, i}(U(n))_{\mu}^{+1} \mid \mu \in I_{n}^{0}\right\} \cup\left\{X_{\mathrm{YM}}^{\ell, i}(U(n))_{\mu}^{-1} \mid \mu \in I_{n}^{0}\right\} .
$$

(ii) If $n$ is even, the connected components of $X_{\mathrm{YM}}^{\ell, i}(U(n))=\operatorname{Hom}\left(\Gamma_{\mathbb{R}}\left(\Sigma_{i}^{\ell}\right)\right.$, $U(n))$ are

$$
\begin{aligned}
& \left\{X_{\mathrm{YM}}^{\ell, i}(U(n))_{\mu}^{+1} \mid \mu \in I_{n}^{0}\right\} \cup\left\{X_{\mathrm{YM}}^{\ell, i}(U(n))_{\mu}=X_{\mathrm{YM}}^{\ell, i}(U(n))_{\mu}^{+1} \mid \mu \in I_{n}^{i,+}\right\} \\
& \cup\left\{X_{\mathrm{YM}}^{\ell, i}(U(n))_{\mu}^{-1} \mid \mu \in I_{n}^{0}\right\} \cup\left\{X_{\mathrm{YM}}^{\ell, i}(U(n))_{\mu}=X_{\mathrm{YM}}^{\ell, i}(U(n))_{\mu}^{-1} \mid \mu \in I_{n}^{i,-}\right\} .
\end{aligned}
$$

Proposition 7.3. Let $\mu=(\nu, \underbrace{0, \ldots, 0}_{n_{0}}, \tau_{0}(\nu)) \in I_{n}$, where

$$
\begin{gathered}
\nu \in I_{n^{\prime}, k}, n^{\prime}, n_{0} \geq 0,2 n^{\prime}+n_{0}=n \\
\nu=(\underbrace{\frac{k_{1}}{n_{1}}, \ldots, \frac{k_{1}}{n_{1}}}_{n_{1}}, \ldots, \underbrace{\frac{k_{m}}{n_{m}}, \ldots, \frac{k_{m}}{n_{m}}}_{n_{m}}), \quad \frac{k_{1}}{n_{1}}>\cdots>\frac{k_{m}}{n_{m}}>0 .
\end{gathered}
$$

(i) If $n_{0}>0$, then for $i=1,2$, we have a homeomorphism

$$
\begin{aligned}
& X_{\mathrm{YM}}^{\ell, i}(U(n))_{\mu}^{ \pm} / U(n) \cong \\
& \quad X_{\text {flat }}^{\ell, i}\left(U\left(n_{0}\right)\right)^{ \pm(-1)^{n i^{\prime}+k}} / U\left(n_{0}\right) \times \prod_{j=1}^{m}\left(X_{\mathrm{YM}}^{2 \ell+i-1,0}\left(U\left(n_{j}\right)\right)_{\frac{k_{j}}{n_{j}}, \ldots, \frac{k_{j}}{n_{j}}} / U\left(n_{j}\right)\right)
\end{aligned}
$$

and a homotopy equivalence

$$
\begin{aligned}
& \left(X_{\mathrm{YM}}^{\ell, i}(U(n))_{\mu}^{ \pm}\right)^{h U(n)} \sim \\
& \quad\left(X_{\mathrm{flat}}^{\ell, i}\left(U\left(n_{0}\right)\right)^{ \pm(-1)^{n i^{\prime}+k}}\right)^{h U\left(n_{0}\right)} \times \prod_{j=1}^{m}\left(X_{\mathrm{YM}}^{2 \ell+i-1,0}\left(U\left(n_{j}\right)\right)_{\frac{k_{j}}{n_{j}}, \ldots, \frac{k_{j}}{n_{j}}}\right)^{h U\left(n_{j}\right)}
\end{aligned}
$$

(ii) If $n_{0}=0$, then for $i=1,2$, we have a homeomorphism

$$
X_{\mathrm{YM}}^{\ell, i}(U(n))_{\mu} / U(n) \cong \prod_{j=1}^{m}\left(X_{\mathrm{YM}}^{2 \ell+i-1,0}\left(U\left(n_{j}\right)\right)_{\frac{k_{j}}{n_{j}}, \ldots, \frac{k_{j}}{n_{j}}} / U\left(n_{j}\right)\right)
$$


and a homotopy equivalence

$$
X_{\mathrm{YM}}^{\ell, i}(U(n))_{\mu}^{h U(n)} \sim \prod_{j=1}^{m}\left(X_{\mathrm{YM}}^{2 \ell+i-1,0}\left(U\left(n_{j}\right)\right)_{\frac{k_{j}}{n_{j}}, \ldots, \frac{k_{j}}{n_{j}}}\right)^{h U\left(n_{j}\right)} .
$$

\subsection{Equivariant Morse stratification and equivariant Poincaré series}

For $i=1,2$, let $P^{n,+}$ and $P^{n,-}$ be the principal $U(n)$-bundles on $\Sigma_{i}^{\ell}$ with $c_{1}\left(P^{n,+}\right)=0$ and $c_{1}\left(P^{n,-}\right)=1$ in $H^{2}\left(\Sigma_{i}^{\ell} ; \mathbb{Z} / 2 \mathbb{Z}\right) \cong \mathbb{Z} / 2 \mathbb{Z}$, respectively. Let $\mathcal{A}\left(\Sigma_{i}^{\ell}\right)^{n, \pm}$ be the space of connections on $P^{n, \pm} \rightarrow \Sigma_{i}^{\ell}$, and let $\mathcal{N}\left(\Sigma_{i}^{\ell}\right)^{n, \pm}$ denote the space of Yang-Mills $U(n)$-connections on $P^{n, \pm} \rightarrow \Sigma_{i}^{\ell}$. Let $\mathcal{G}^{n, \pm}$ and $\mathcal{G}_{0}^{n, \pm}$ denote the gauge group and based gauge group, respectively. $\mathcal{N}\left(\Sigma_{i}^{\ell}\right)_{0}^{1, \pm}$ is the space of flat $U(1)$-connections on $P^{1, \pm} \rightarrow \Sigma_{i}^{\ell}$, and we have (see $[10,11])$

$$
X_{\text {flat }}^{\ell, i}(U(1))^{ \pm 1}=\mathcal{N}\left(\Sigma_{i}^{\ell}\right)_{0}^{1, \pm} / \mathcal{G}_{0}^{1, \pm}, \quad X_{\text {flat }}^{\ell, i}(U(1))^{ \pm 1} / U(1)=\mathcal{N}\left(\Sigma_{i}^{\ell}\right)_{0}^{1, \pm} / \mathcal{G}^{1, \pm}
$$

Since $c_{1}(P)=c_{1}(\operatorname{det}(P)) \in H^{2}(\Sigma ; \mathbb{Z} / 2 \mathbb{Z})$, we have

$$
\begin{gathered}
\operatorname{Hom}\left(\Gamma_{\mathbb{R}}\left(\Sigma_{i}^{\ell}\right), U(n)\right)=X_{\mathrm{YM}}^{\ell, i}(U(n))^{+1} \cup X_{\mathrm{YM}}^{\ell, i}(U(n))^{-1} \\
X_{\mathrm{YM}}^{\ell, i}(U(n))^{ \pm 1}=\mathcal{N}\left(\Sigma_{i}^{\ell}\right)^{n, \pm} / \mathcal{G}_{0}^{n, \pm}, \quad X_{\mathrm{YM}}^{\ell, i}(U(n))^{ \pm 1} / U(n)=\mathcal{N}\left(\Sigma_{i}^{\ell}\right)^{n, \pm} / \mathcal{G}^{n, \pm} .
\end{gathered}
$$

The connected components of $X_{\mathrm{YM}}^{\ell, i}(U(n))^{ \pm}$are

$$
\left\{X_{\mathrm{YM}}^{\ell, i}(U(n))_{\mu}^{ \pm 1} \mid \mu \in I_{n}^{0}\right\} \cup\left\{X_{\mathrm{YM}}^{\ell, i}(U(n))_{\mu} \mid \mu \in I_{n}^{i, \pm}\right\} .
$$

When $n$ is odd, both $I_{n}^{i,+}$ and $I_{n}^{i,-}$ are empty. Given $\mu \in I_{n}^{0}$, let $\mathcal{N}\left(\Sigma_{i}^{\ell}\right)_{\mu}^{ \pm}$be the preimage of $X_{\mathrm{YM}}^{\ell, i}(U(n))_{\mu}^{ \pm 1}$ under the projection

$$
\mathcal{N}\left(\Sigma_{i}^{\ell}\right)^{n, \pm} \longrightarrow \mathcal{N}\left(\Sigma_{i}^{\ell}\right)^{n, \pm} / \mathcal{G}_{0}^{n, \pm}=X_{\mathrm{YM}}^{\ell, i}(U(n))^{ \pm 1}
$$

Given $\mu \in I_{n}^{i, \pm}$, let $\mathcal{N}\left(\Sigma_{i}^{\ell}\right)_{\mu}$ be the preimage of $X_{\mathrm{YM}}^{\ell, i}(U(n))_{\mu}$ under the projection

$$
\mathcal{N}\left(\Sigma_{i}^{\ell}\right)^{n, \pm} \longrightarrow \mathcal{N}\left(\Sigma_{i}^{\ell}\right)^{n, \pm} / \mathcal{G}_{0}^{n, \pm}=X_{\mathrm{YM}}^{\ell, i}(U(n))^{ \pm 1}
$$

In other words,

$$
\begin{aligned}
\mathcal{N}\left(\Sigma_{i}^{\ell}\right)_{\mu}^{ \pm} / \mathcal{G}_{0}^{n, \pm} & \cong X_{\mathrm{YM}}^{\ell, i}(U(n))_{\mu}^{ \pm 1}, \quad \mu \in I_{n}^{0} \\
\mathcal{N}\left(\Sigma_{i}^{\ell}\right)_{\mu} / \mathcal{G}_{0}^{n, \pm} & \cong X_{\mathrm{YM}}^{\ell, i}(U(n))_{\mu}, \quad \mu \in I_{n}^{i, \pm}
\end{aligned}
$$


In particular,

$$
\mathcal{N}\left(\Sigma_{i}^{\ell}\right)_{n}^{ \pm \ldots, \ldots, 0}=\mathcal{N}\left(\Sigma_{i}^{\ell}\right)_{0}^{n, \pm}
$$

is the space of flat connections on $P^{n, \pm} \rightarrow \Sigma_{i}^{\ell}$. The Yang-Mills functional achieves its absolute minimum 0 on $\mathcal{N}\left(\Sigma_{i}^{\ell}\right)_{0}^{n, \pm}$. The moduli space of gauge equivalence classes of flat connections on $P^{n, \pm} \rightarrow \Sigma_{i}^{\ell}$ is given by

$$
\mathcal{M}\left(\Sigma_{i}^{\ell}, P^{n, \pm}\right)=\mathcal{N}\left(\Sigma_{i}^{\ell}\right)_{0}^{n, \pm} / \mathcal{G}^{n, \pm} \cong V_{s s}\left(\Sigma_{i}^{\ell}, P^{n, \pm}\right) / U(n),
$$

where

$$
V_{s s}\left(\Sigma_{i}^{\ell}, P^{n, \pm}\right)=X_{\text {flat }}^{\ell, i}(U(n))^{ \pm}=\mathcal{N}\left(\Sigma_{i}^{\ell}\right)_{0}^{n, \pm} / \mathcal{G}_{0}^{n, \pm} .
$$

Now assume $\chi\left(\Sigma_{i}^{\ell}\right)=2-2 \ell-i<0$. Let $\pi: \tilde{\Sigma} \rightarrow \Sigma$ be the orientable double cover. Then $\pi^{*} P^{n, \pm}=P^{n, 0} \cong \tilde{\Sigma} \times U(n)$. There are involutions $\tau^{ \pm}$: $P^{n, 0} \rightarrow P^{n, 0}$ which cover the deck transformation $\tau: \tilde{\Sigma} \rightarrow \tilde{\Sigma}$ such that $P^{n, 0} /$ $\tau^{ \pm}=P^{n, \pm}$. Let $\mathcal{A}(\Sigma)^{n, \pm}$ denote the space of connections on $P^{n, \pm} \rightarrow \Sigma$, and let $\mathcal{A}(\tilde{\Sigma})^{n, 0}$ denote the space of connections on $P^{n, 0} \rightarrow \tilde{\Sigma}$. Then

$$
\mathcal{A}(\Sigma)^{n, \pm} \cong\left(\mathcal{A}(\tilde{\Sigma})^{n, 0}\right)^{\tau^{ \pm}} .
$$

Given $\mu \in I_{n}^{0}$ (resp. $\mu \in I_{n}^{i,+} \cup I_{n}^{i,-}$ ), let $\mathcal{A}(\Sigma)_{\mu}^{ \pm}$(resp. $\left.\mathcal{A}(\Sigma)_{\mu}\right)$ be the stable manifold of the critical set $\mathcal{N}(\Sigma)_{\mu}^{ \pm}$(resp. $\left.\mathcal{N}(\Sigma)_{\mu}\right)$ of the Yang-Mills functional on $\mathcal{A}(\Sigma)^{ \pm}$. Then for $i=1,2$,

$$
\mathcal{A}\left(\Sigma_{i}^{\ell}\right)^{n, \pm}=\bigcup_{\mu \in I_{n}^{0}} \mathcal{A}\left(\Sigma_{i}^{\ell}\right)_{\mu}^{ \pm} \cup \bigcup_{\mu \in I_{n}^{i, \pm}} \mathcal{A}\left(\Sigma_{i}^{\ell}\right)_{\mu}
$$

is the $\mathcal{G}$-equivariant Morse stratification of $\mathcal{A}\left(\Sigma_{i}^{\ell}\right)^{n, \pm}$ given by the Yang-Mills functional.

We have

$$
\mathcal{N}\left(\Sigma_{i}^{\ell}\right)_{\mu}^{ \pm} \subset\left(\mathcal{A}(\tilde{\Sigma})_{\mu}^{n, 0}\right)^{\tau^{ \pm}} \text {for } \mu \in I_{n}^{0}, \quad \mathcal{N}\left(\Sigma_{i}^{\ell}\right)_{\mu} \subset\left(\mathcal{A}(\tilde{\Sigma})_{\mu}^{n, 0}\right)^{\tau^{ \pm}} \text {for } \mu \in I_{n}^{i, \pm} .
$$

By results in [1] and [6], $\mathcal{A}_{\mu}$ is a complex submanifold of $\mathcal{A}$. We also know that $\tau^{ \pm}$induces anti-holomorphic involution on $\mathcal{A}$. By Proposition 5.1, for $\mu \in I_{n}^{0}$, we have

$$
\begin{aligned}
\operatorname{codim}_{\mathbb{R}}\left(\mathcal{A}(\Sigma)_{\mu}^{ \pm}, \mathcal{A}(\Sigma)^{n, \pm}\right) & =\operatorname{codim}_{\mathbb{C}}\left(\mathcal{A}(\tilde{\Sigma})_{\mu}, \mathcal{A}(\tilde{\Sigma})^{n, 0}\right) \\
& =\sum_{\alpha<\beta}\left(\mu_{\alpha}-\mu_{\beta}-\chi\left(\Sigma_{i}^{\ell}\right)\right) .
\end{aligned}
$$

The above formula also holds for $\mu \in I_{n}^{i, \pm}$. 
We have the following equivalent equivariant pairs for the purpose of equivariant (singular) cohomology:

$$
\begin{aligned}
& \left(\mathcal{A}\left(\Sigma_{i}^{\ell}\right)_{\mu}^{ \pm}, \mathcal{G}^{n, \pm}\right) \sim\left(\mathcal{N}\left(\Sigma_{i}^{\ell}\right)_{\mu}^{ \pm}, \mathcal{G}^{n, \pm}\right) \sim\left(X_{\mathrm{YM}}^{\ell, i}(U(n))_{\mu}^{ \pm 1}, U(n)\right), \quad \mu \in I_{n}^{0} \\
& \left(\mathcal{A}\left(\Sigma_{i}^{\ell}\right)_{\mu}, \mathcal{G}^{n, \pm}\right) \sim\left(\mathcal{N}\left(\Sigma_{i}^{\ell}\right)_{\mu}, \mathcal{G}^{n, \pm}\right) \sim\left(X_{\mathrm{YM}}^{\ell, i}(U(n))_{\mu}, U(n)\right), \quad \mu \in I_{n}^{i, \pm}
\end{aligned}
$$

In other words, we have the following homotopy equivalences of homotopic orbit spaces:

$$
\begin{aligned}
& \left(\mathcal{A}\left(\Sigma_{i}^{\ell}\right)_{\mu}^{ \pm}\right)^{h \mathcal{G}^{n, \pm}} \sim\left(\mathcal{N}\left(\Sigma_{i}^{\ell}\right)_{\mu}^{ \pm}\right)^{h \mathcal{G}^{n, \pm}} \sim\left(X_{\mathrm{YM}}^{\ell, i}(U(n))_{\mu}^{ \pm 1}\right)^{h U(n)}, \quad \mu \in I_{n}^{0} \\
& \mathcal{A}\left(\Sigma_{i}^{\ell}\right)_{\mu}{ }^{h \mathcal{G}^{n, \pm}} \sim \mathcal{N}\left(\Sigma_{i}^{\ell}\right)_{\mu}{ }^{h \mathcal{G}^{n, \pm}} \sim X_{\mathrm{YM}}^{\ell, i}(U(n))_{\mu}{ }^{h U(n)}, \quad \mu \in I_{n}^{i, \pm} .
\end{aligned}
$$

Together with the reduction Proposition 7.3, we have

Theorem 7.4. Let $i=1,2$, and let $K$ be a field. Given $\mu=(\nu, \underbrace{0, \ldots, 0}_{n_{0}}$, $\left.\tau_{0}(\nu)\right) \in I_{n}$, where $\nu \in I_{n^{\prime}, k}, n^{\prime}, n_{0} \geq 0,2 n^{\prime}+n_{0}=n$,

$$
\nu=(\underbrace{\frac{k_{1}}{n_{1}}, \ldots, \frac{k_{1}}{n_{1}}}_{n_{1}}, \ldots, \underbrace{\frac{k_{m}}{n_{m}}, \ldots, \frac{k_{m}}{n_{m}}}_{n_{m}}), \quad \frac{k_{1}}{n_{1}}>\cdots>\frac{k_{m}}{n_{m}}>0
$$

we have the following identities.

(i) $n_{0}>0 \Longleftrightarrow \mu \in I_{n}^{0}$ :

$$
\begin{aligned}
& H_{\mathcal{G}}^{*}\left(\mathcal{A}\left(\Sigma_{i}^{\ell}\right)_{\mu}^{ \pm} ; K\right) \cong H_{\mathcal{G}}^{*}\left(\mathcal{N}\left(\Sigma_{i}^{\ell}\right)_{\mu}^{ \pm} ; K\right) \cong H_{U(n)}^{*}\left(X_{\mathrm{YM}}^{\ell, i}(U(n))_{\mu}^{ \pm} ; K\right) \\
& \quad \cong H_{U\left(n_{0}\right)}^{*}\left(V_{s s}\left(\Sigma_{i}^{\ell}, P^{n_{0}, \pm(-1)^{n^{\prime} i+k}}\right) ; K\right) \\
& \quad \otimes \bigotimes_{j=1}^{m} H_{U\left(n_{j}\right)}^{*}\left(V_{s s}\left(\Sigma_{0}^{2 \ell+i-1}, P^{n_{j}, k_{j}}\right) ; K\right) \\
& P_{t}^{\mathcal{G}}\left(\mathcal{A}\left(\Sigma_{i}^{\ell}\right)_{\mu}^{ \pm} ; K\right)=P_{t}^{\mathcal{G}}\left(\mathcal{N}\left(\Sigma_{i}^{\ell}\right)_{\mu}^{ \pm} ; K\right)=P_{t}^{U(n)}\left(X_{\mathrm{YM}}^{\ell, i}(U(n))_{\mu}^{ \pm 1} ; K\right) \\
& \quad=P_{t}^{U\left(n_{0}\right)}\left(V_{s s}\left(\Sigma_{i}^{\ell}, P^{n_{0}, \pm(1)^{n^{\prime} i+k}}\right) ; K\right) \cdot \prod_{j=1}^{m} P_{t}^{U\left(n_{j}\right)}\left(V_{s s}\left(\Sigma_{0}^{2 \ell+i-1}, P^{n_{j}, k_{j}}\right) ; K\right) .
\end{aligned}
$$


(ii) $n_{0}=0 \Leftrightarrow \mu \in I_{n}^{i, \pm}$ :

$$
\begin{aligned}
& H_{\mathcal{G}}^{*}\left(\mathcal{A}\left(\Sigma_{i}^{\ell}\right)_{\mu} ; K\right) \cong H_{\mathcal{G}}^{*}\left(\mathcal{N}\left(\Sigma_{i}^{\ell}\right)_{\mu} ; K\right) \cong H_{U(n)}^{*}\left(X_{\mathrm{YM}}^{\ell, i}(U(n))_{\mu} ; K\right) \\
& \quad \cong \bigotimes_{j=1}^{m} H_{U\left(n_{j}\right)}^{*}\left(V_{s s}\left(\Sigma_{0}^{2 \ell+i-1}, P^{n_{j}, k_{j}}\right) ; K\right) \\
& P_{t}^{\mathcal{G}}\left(\mathcal{A}\left(\Sigma_{i}^{\ell}\right)_{\mu} ; K\right)=P_{t}^{\mathcal{G}}\left(\mathcal{N}\left(\Sigma_{i}^{\ell}\right)_{\mu} ; K\right)=P_{t}^{U(n)}\left(X_{\mathrm{YM}}^{\ell, i}(U(n))_{\mu} ; K\right) \\
& \quad=\prod_{j=1}^{m} P_{t}^{U\left(n_{j}\right)}\left(V_{s s}\left(\Sigma_{0}^{2 \ell+i-1}, P^{n_{j}, k_{j}}\right) ; K\right)
\end{aligned}
$$

Example 7.5. $n=2$. For $\Sigma_{1}^{\ell}$,

$$
\begin{aligned}
& I_{n}^{1,+}=\{(0,0)\} \cup\left\{(2 r-1,1-2 r) \mid r \in \mathbb{Z}_{>0}\right\} \\
& I_{n}^{1,-}=\{(0,0)\} \cup\left\{(2 r,-2 r) \mid r \in \mathbb{Z}_{>0}\right\} .
\end{aligned}
$$

$\operatorname{For} \Sigma_{2}^{\ell}$

$$
\begin{aligned}
& I_{n}^{2,+}=\{(0,0)\} \cup\left\{(2 r,-2 r) \mid r \in \mathbb{Z}_{>0}\right\} \\
& I_{n}^{2,-}=\{(0,0)\} \cup\left\{(2 r-1,1-2 r) \mid r \in \mathbb{Z}_{>0}\right\}
\end{aligned}
$$

In other words, the $\mathcal{G}$-equivariant strata of $\mathcal{A}\left(P^{2,+}\right)\left(\right.$ resp. $\left.\mathcal{A}\left(P^{2,-}\right)\right)$ are $\left\{\mathcal{A}\left(\Sigma_{i}^{\ell}\right)_{\mu} \mid \mu \in I_{2}^{i,+}\right.$ (resp. $\left.\left.I_{2}^{i,-}\right)\right\}$. The codimension of each stratum is

$$
d_{2 r,-2 r}=4 r+2 \ell+i-2, \quad d_{2 r-1,1-2 r}=4 r+2 \ell+i-4 .
$$

The equivariant Poincaré series for stratum $\mu=(r,-r)$ is

$$
\begin{aligned}
P_{t}^{\mathcal{G}}\left(\mathcal{A}\left(\Sigma_{i}^{\ell}\right)_{r,-r}\right) & =P_{t}^{U(2)}\left(X_{\mathrm{YM}}^{\ell, i}(U(2))_{r,-r}\right)=P_{t}^{U(1)}\left(X_{\mathrm{YM}}^{2 \ell+i-1,0}(U(1))_{r}\right) \\
& =P_{t}^{U(1)}\left(U(1)^{2(2 \ell+i-1)}\right)=\frac{(1+t)^{2(2 \ell+i-1)}}{1-t^{2}}
\end{aligned}
$$

Example 7.6. $n=3$. Since $n$ is odd, $n_{0}=1$ or 3 . Thus,

$$
I_{n}^{0}=\{(0,0,0)\} \cup\left\{(r, 0,-r) \mid r \in \mathbb{Z}_{>0}\right\}
$$


The $\mathcal{G}$-equivariant strata of $\mathcal{A}\left(P^{3, \pm}\right)$ are $\left\{\mathcal{A}_{\mu}^{ \pm} \mid \mu \in I_{3}^{0}\right\}$. The codimension of each stratum is

$$
d_{r, 0,-r}=4 r+3(2 \ell+i-2)
$$

The equivariant Poincaré series for stratum $\mu=(r, 0,-r)$ is

$$
\begin{aligned}
P_{t}^{U(3)} & \left(X_{\mathrm{YM}}^{\ell, i}(U(3))_{r, 0,-r}^{ \pm 1}\right) \\
= & P_{t}^{U(1)}\left(X_{\text {flat }}^{\ell, i}(U(1))^{ \pm(-1)^{i+r}}\right) P_{t}^{U(1)}\left(X_{\mathrm{YM}}^{2 \ell+i-1,0}(U(1))_{r}\right) \\
= & P_{t}^{U(1)}\left(U(1)^{2 \ell+i-1}\right) P_{t}^{U(1)}\left(U(1)^{2(2 \ell+i-1)}\right)=\frac{(1+t)^{3(2 \ell+i-1)}}{\left(1-t^{2}\right)^{2}}
\end{aligned}
$$

\section{Acknowledgments}

This project was motivated by Raoul Bott's, Kenji Fukaya's, and Lisa Jeffrey's questions on betti numbers of moduli spaces of flat connections over nonorientable surfaces. We thank Paul Goerss, Victor Guillemin, Frances Kirwan, Lisa Jeffrey, Jun Li, Eckhard Meinrenken, Mudumbai S. Narasimhan, Daniel Ramras, Paul Selick, Reyer Sjamaar, Jonathan Weitsman, Graeme Wilkin, Christopher Woodward, and Siye Wu for helpful conversations. We thank Lisa Jeffrey, Eckhard Meinrenken, and Christopher Woodward for comments and suggestions on our draft. The first author was partially supported by Natural Sciences and Engineering Research Council of Canada Postdoctoral Fellowship.

\section{References}

[1] M.F. Atiyah and R. Bott, The Yang-Mills equations over Riemann surfaces, Philos. Trans. Roy. Soc. London Ser. A 308(1505) (1983), 523-61.

[2] A. Alekseev, E. Meinrenken and C. Woodward, Duistermaat-Heckman measures and moduli spaces of flat bundles over surfaces, Geom. Funct. Anal. 12(1) (2002), 1-31.

[3] D.M. Austin and P.J. Braam, Morse-Bott theory and equivariant cohomology, The Floer Memorial Volume, Progr. Math., 133, Birkhäuser, Basel, 1995, 123-183. 
[4] T. Baird, The moduli space of flat SU(2)-connections over a nonorientable surface, arXiv:0806.1975.

[5] D. Biss, V. Guillemin and T.S. Holm, The mod 2 cohomology of fixed point sets of anti-symplectic involutions, Adv. Math. 185 (2) (2004), 370-399.

[6] G.D. Daskalopoulos, The topology of the space of stable bundles on a compact Riemann surface, J. Differential Geom. 36 (1992), 699-746.

[7] R.F. Goldin and T.S. Holm, Real loci of symplectic reductions, Trans. Amer. Math. Soc. 356 (11) (2004), 4623-4642.

[8] N.-K. Ho, The real locus of an involution map on the moduli space of flat connections on a Riemann surface, Int. Math. Res. Not. 61 (2004), 3263-3285.

[9] N.-K. Ho and C.-C.M. Liu, On the connectedness of moduli spaces of flat connections over compact surfaces, Canad. J. Math. 56 (6) (2004), 1228-1236.

[10] - Connected components of the space of surface group representations, Int. Math. Res. Not. 44 (2003), 2359-2372.

[11] - Connected components of the space of surface group representations II, Int. Math. Res. Not. 16 (2005), 959-979.

[12] - Yang-Mills connections on orientable and nonorientable surfaces, arXiv:0707.0258, to appear in Memoirs of the American Mathematical Society.

[13] D. Huybrechts and M. Lehn, The Geometry of moduli spaces of sheaves, Aspects of Mathematics, E31, Friedr. Vieweg \& Sohn, Braunschweig, 1997.

[14] G. Harder and M.S. Narasimhan, On the cohomology groups of moduli spaces of vector bundles on curves, Math. Ann. 212 (1974/75), 215-248.

[15] L. Jeffrey, Extended moduli spaces of flat connections on Riemann surfaces, Math. Ann. 298 (1994), 667-692.

[16] Y.-H. Kiem, Intersection cohomology of representation spaces of surface groups, Internat. J. Math. 17(2) (2006), 169-182. 
[17] F. Kirwan, Cohomology of quotients in symplectic and algebraic geometry, Princeton University Press, 1984.

[18] - On spaces of maps from Riemann surfaces to Grassmannians and applications to the cohomology of moduli of vector bundles, Ark. Math. 24 (1986), 221-275.

[19] - On the homology of compactifications of moduli spaces of vector bundles over a Riemann surface, Proc. London Math. Soc. 53 (1986), 237-266.

[20] - The cohomology rings of moduli spaces of bundles over Riemann surfaces J. Amer. Math. Soc. 5 (1992), 853-906.

[21] S. Kobayashi, Differential geometry of complex vector bundles, Publications of the Mathematical Society of Japan, 15, Kanô Memorial Lectures, 5, Princeton University Press, Princeton, NJ; Iwanami Shoten, Tokyo, 1987.

[22] G. Laumon and M. Rapoport, The Langlands lemma and the betti numbers of stacks fo G-bundles on a curve, Internat. J. Math. 7(1) (1996), 29-45.

[23] D. Mumford, J. Fogarty and F. Kirwan, Geometric invariant theory, 3rd edition, Springer-Verlag, 1994.

[24] J. Råde, On the Yang-Mills heat equation in two and three dimensions, J. Reine Angew. Math. 431 (1992), 123-163.

[25] A. Ramanathan, Stable principal bundles on a compact Riemann surface, Math. Ann. 213 (1975), 129-152.

[26] D. Ramras, Yang-Mills theory over surfaces and the Atiyah-Segal theorem, arXiv:0710.0681.

[27] - The Yang-Mills stratification for surfaces revisited, arXiv:0805.2587v2.

[28] K. Uhlenbeck and S.-T. Yau, On the existence of Hermitian-YangMills connections in stable vector bundles, Frontiers of the Mathematical Sciences, 1985 (New York, 1985), Comm. Pure Appl. Math. 39(S) (1986), suppl., S257-S293.

[29] S. Wang, A Narasimhan-Seshadri-Donaldson correspondence over non-orientable surfaces, Forum Math. 8(4) (1996), 461-474. 
[30] C. Woodward, Localization for the norm-square of the moment map and the two-dimensional Yang-Mills integral, J. Symplectic Geom. 3(1) (2005), 17-54.

Department of Mathematics

National Cheng-Kung University

AND

National Center for Theoretical Sciences (South)

TAINAN 701

TAIWAN

E-mail address: nankuo@mail.ncku.edu.tw

Department of Mathematics

COLUMBia UNIVERSITY

New YORK

NY 10027

USA

E-mail address: ccliu@math.columbia.edu

Received January 13, 2008 
